\title{
Projective multiplets and hyperkähler cones in conformal supergravity
}

\section{Daniel Butter}

Nikhef Theory Group,

Science Park 105, 1098 XG Amsterdam, The Netherlands

E-mail: dbutter@nikhef.nl

ABSTRACT: Projective superspace provides a natural framework for the construction of actions coupling hypermultiplets to conformal supergravity. We review how the off-shell actions are formulated in superspace and then discuss how to eliminate the infinite number of auxiliary fields to produce an on-shell $\mathcal{N}=2$ supersymmetric sigma model, with the target space corresponding to a generic $4 n$-dimensional hyperkähler cone. We show how the component action coupling the hypermultiplets to conformal supergravity may be constructed starting from curved superspace. The superspace origin of the geometric data - the hyperkähler potential, complex structures, and any gauged isometries - is also addressed.

KEywords: Extended Supersymmetry, Superspaces, Sigma Models

ARXIV EPRINT: 1410.3604

Dedicated to the memory of Bruno Zumino 


\section{Contents}

1 Introduction 2

2 A review of hyperkähler geometry from flat projective superspace $\quad 6$

3 Hyperkähler geometry and on-shell $\mathcal{N}=2$ superfields $\quad 8$

$\begin{array}{ll}3.1 \text { Hyperkähler geometry } & 10\end{array}$

3.2 Gauged isometries from projective superspace 11

$\begin{array}{lll}3.3 & \text { Superconformal isometries } & 13\end{array}$

$\begin{array}{lll}3.4 & \text { Supersymmetry and fermion transformations } & 14\end{array}$

$\begin{array}{lll}3.5 & \mathrm{Sp}(n) \text {-covariant formulation and summary } & 15\end{array}$

4 Building blocks of a component reduction $\quad 16$

$\begin{array}{ll}\text { 4.1 Component expansions of the projective multiplets } & 17\end{array}$

$\begin{array}{lll}4.2 & \text { Auxiliary field equations of motion } & 19\end{array}$

$\begin{array}{lll}4.3 & \text { Some relevant quantities } 20\end{array}$

4.3.1 Hyperkähler two-forms and their pullbacks 21

4.3.2 Components of the $\mathcal{N}=2$ moment map 22

4.3.3 Hyperkähler cone potential and hyperkähler one-forms 22

5 The component action in rigid projective superspace $\quad 23$

5.1 Rigid ungauged terms 24

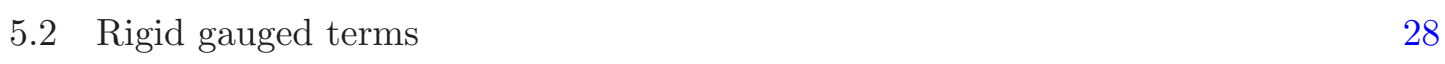

6 The component action in curved projective superspace $\quad 30$

$\begin{array}{lll}6.1 & \text { Remaining evaluation of } T_{0} & 31\end{array}$

6.2 Simplification of the total covariant derivative 33

6.3 Gravitino terms 33

$\begin{array}{lll}6.4 & \text { Final result } & 36\end{array}$

$\begin{array}{lll}7 & \text { Conclusion } & 37\end{array}$

A Vector multiplet conventions and supersymmetry transformations $\quad 39$

B Vanishing of a pure arctic action and the remainder terms in eq. (6.22) 41 


\section{Introduction}

The hypermultiplet is the most general on-shell matter multiplet with rigid $\mathcal{N}=2$ supersymmetry in four dimensions. It consists of a complex doublet of scalar fields and a pair of Weyl fermions (equivalently, a single Dirac fermion), whose supersymmetry transformations close only on the equations of motion. For this reason, specifying the hypermultiplet is equivalent to specifying the action, or equivalently, specifying the target space of the sigma model parametrized by the hypermultiplet scalars. It has long been known that such target spaces must be hyperkähler manifolds [1, 2]. For locally supersymmetric theories - by which one usually means those coupled to supergravity with a canonically normalized Einstein-Hilbert Lagrangian - the target space must instead be quaternion-Kähler [3]. This distinction recalls that between rigid $\mathcal{N}=1$ theories, where the target space is Kähler [4], and their locally supersymmetric counterparts, which must be Hodge-Kähler [5]. ${ }^{1}$

It is known that off-shell formulations of the hypermultiplet, employing $\mathcal{N}=2$ superspace and involving (an infinite number of) auxiliary fields, provide a means to generate the required target space geometry from some unconstrained generating function. To understand this better, let us elaborate first on the rigid $\mathcal{N}=1$ case. Recall that the chiral multiplet $\phi$, the natural $\mathcal{N}=1$ cousin of the hypermultiplet, admits a simple off-shell representation $\left\{\phi, \zeta_{\alpha}, F\right\}$ with a single complex auxiliary field. (We employ a convention where a single symbol $\phi$ may stand for both a superfield and its lowest component.) Zumino showed that the most general two-derivative function of off-shell chiral multiplets $\phi^{\mathrm{a}}$ is described by a superspace integral [4]

$$
\int \mathrm{d}^{4} x \mathrm{~d}^{2} \theta \mathrm{d}^{2} \bar{\theta} K(\phi, \bar{\phi})
$$

for an arbitrary real function $K .^{2}$ When the auxiliary fields $F^{\text {a }}$ are eliminated, the component Lagrangian takes a sigma model form ${ }^{3}$

$$
\begin{aligned}
\mathcal{L} & =-g_{\mathrm{a} \overline{\mathrm{b}}} \partial_{m} \phi^{\mathrm{a}} \partial^{m} \bar{\phi}^{\overline{\mathrm{b}}}-\frac{i}{4} g_{\mathrm{a} \overline{\mathrm{b}}}\left(\zeta^{\mathrm{a}} \sigma^{m} \widehat{\mathcal{D}}_{m} \bar{\zeta}^{\overline{\mathrm{b}}}+\bar{\zeta}^{\overline{\mathrm{b}}} \bar{\sigma}^{m} \widehat{\mathcal{D}}_{m} \zeta^{\mathrm{a}}\right)+\frac{1}{16} R_{\mathrm{a} \overline{\mathrm{a}} \overline{\mathrm{b}}}\left(\zeta^{\mathrm{a}} \zeta^{\mathrm{b}}\right)\left(\bar{\zeta}^{\mathrm{a}} \bar{\zeta}^{\overline{\mathrm{b}}}\right), \\
\zeta_{\alpha}^{\mathrm{a}} & :=D_{\alpha} \phi^{\mathrm{a}}, \quad \widehat{\mathcal{D}}_{m} \zeta^{\mathrm{a}}:=\partial_{m} \zeta^{\mathrm{a}}+\Gamma_{\mathrm{bc}}{ }^{\mathrm{a}} \partial_{m} \phi^{\mathrm{b}} \zeta^{\mathrm{c}} .
\end{aligned}
$$

The metric is determined in terms of the function $K, g_{\mathrm{a} \overline{\mathrm{b}}}:=\partial_{\mathrm{a}} \partial_{\overline{\mathrm{b}}} K$; of course, this is the well-known Kähler metric - in using chiral multiplets we have automatically diagonalized the complex structure - and this construction gives the explicit proof that $\mathcal{N}=1$ sigma models in four dimensions must possess a Kähler target space geometry [4]. The key point is that the general off-shell supersymmetric action (1.1) provides both the means to construct the on-shell action and the generating function for the target space geometry.

This narrative can be repeated for $\mathcal{N}=2$ theories. There one has two complementary formulations of a general off-shell hypermultiplet, depending on whether one uses harmonic

\footnotetext{
${ }^{1}$ See also [6-9] for a new perspective on these results.

${ }^{2}$ For now we ignore the possibility of a superpotential or gauged isometries.

${ }^{3}$ The fermion $\zeta_{\alpha}^{\mathrm{a}}$ is normalized here in an unconventional way. We use the same normalization for $\mathcal{N}=2$ models, following [10].
} 
superspace $[10,11]$ or projective superspace [12-14]. In harmonic superspace, which augments $\mathcal{N}=2$ superspace with an auxiliary $S^{2}$ manifold with harmonic coordinates $u^{i \pm}$, one has $n$ pseudoreal superfields $q^{\mathrm{a}+}, \widetilde{q^{\mathrm{a}+}}=-q^{\mathrm{b}+} \Omega_{\mathrm{ba}}$ where $\Omega$ is the canonical symplectic form of $\mathrm{Sp}(\mathrm{n})$, obeying the analyticity condition

$$
u_{i}^{+} D_{\underline{\alpha}}^{i} q^{\mathrm{a}+} \equiv D_{\underline{\alpha}}^{+} q^{\mathrm{a}+}=0, \quad D_{\underline{\alpha}}^{i} \equiv\left(D_{\alpha}^{i}, \bar{D}_{\dot{\alpha}}^{i}\right) .
$$

This $q^{+}$hypermultiplet is the natural off-shell matter multiplet of harmonic superspace and is defined globally on the $S^{2}$. The most general two-derivative action is

$$
\int \mathrm{d} u \int \mathrm{d}^{4} x \mathrm{~d}^{4} \theta^{+}\left(\frac{1}{2} \Omega_{\mathrm{ab}} q^{\mathrm{a}+} D^{++} q^{\mathrm{b}+}+H^{+4}\left(q^{+}, u^{ \pm}\right)\right)
$$

with $H^{+4}\left(q^{+}, u^{ \pm}\right)$an arbitrary real function $[15,16]$. The striking resemblance to a Hamiltonian system was explained in [16] (see also [17]), where it was shown that any hyperkähler manifold locally possesses a Hamiltonian structure.

For projective superspace, one uses the same auxiliary manifold $\mathbb{C} P^{1} \cong S^{2}$ as in harmonic superspace, but superfields are taken to be holomorphic on an open domain of $\mathbb{C} P^{1}$ rather than globally defined on $S^{2}$, which is the main distinction between the two approaches. ${ }^{4}$ We denote the auxiliary coordinates by $v^{i \pm}$ to distinguish them from harmonic superspace. Sigma models are described by $n$ complex arctic superfields $\Upsilon^{I+}$ and their antarctic conjugates $\breve{\Upsilon}^{I+}=\widetilde{\Upsilon^{I+}}$. These are Grassmann analytic, $D_{\alpha}^{+} \Upsilon^{I+}=0$, and holomorphic on an open domain of $\mathbb{C} P^{1}, D^{++} \Upsilon^{I+}=0$. Arctic multiplets are taken to be holomorphic near the north pole and antarctics are holomorphic near the south pole. The combination of $\Upsilon^{I+}$ and $\breve{\Upsilon}^{\bar{I}+}$, collectively known as a polar multiplet, serves as the general off-shell matter multiplet in projective superspace, and the most general two-derivative action is given by [13] (see also [23] for a recent discussion)

$$
-\frac{1}{2 \pi} \oint_{\mathcal{C}} v_{i}^{+} \mathrm{d} v^{i+} \int \mathrm{d}^{4} x \mathrm{~d}^{4} \theta^{+} \mathcal{F}^{++}\left(\Upsilon^{+}, \breve{\Upsilon}^{+}, v^{i+}\right)
$$

involving an arbitrary real function $\mathcal{F}^{++}$that is homogeneous of weight two in its parameters, $\mathcal{F}^{++}\left(\lambda \Upsilon^{+}, \lambda \breve{\Upsilon}^{+}, \lambda v^{i+}\right)=\lambda^{2} \mathcal{F}^{++}\left(\Upsilon^{+}, \breve{\Upsilon}^{+}, v^{i+}\right)$. This function can be interpreted as the generating function for symplectic transformations on the hyperkähler manifold [24] (see also the pioneering work of [25] and the recent approach of [26]) and turns out to possess a simple relationship to the harmonic Hamiltonian $H^{+4}$ [27].

In both the projective and harmonic cases, the data necessary to define the hyperkähler geometry are encoded in their respective superspace Lagrangians. In evaluating the component actions, one must find a way to eliminate the infinite number of auxiliary fields. In doing so, one generates a hyperkähler metric and associated complex structures that describe the sigma model parametrized by the physical scalars. In principle this can be done explicitly for a specific generating function, although only certain classes of hyperkähler metrics have been explicitly constructed in this way. Once the auxiliaries are eliminated

\footnotetext{
${ }^{4}$ We follow the projective superspace conventions of [18] (similar to those of [19-22]), to which we refer for definitions, notations, and further references.
} 
and the geometric data constructed, the component action takes its final form. These are distinct steps because the component reduction is just as easily done for a general geometry as a specific one, since one can treat the conversion from generating function to hyperkähler geometry completely formally. Our concern in this paper will be with the general action and with the formal elimination of the auxiliaries; we will return to the subject of specific solvable classes in the conclusion.

What about locally supersymmetric theories? In this case, there are two options, depending on whether one couples minimally to conformal supergravity or to Poincaré supergravity with a canonical Einstein-Hilbert term. In the $\mathcal{N}=1$ setting, a sigma model coupled to conformal supergravity is given by

$$
\int \mathrm{d}^{4} x \mathrm{~d}^{2} \theta \mathrm{d}^{2} \bar{\theta} E K(\phi, \bar{\phi}), \quad E=\operatorname{sdet} E_{M}{ }^{A},
$$

except now the superconformal algebra includes both dilatations $(\mathbb{D})$ and chiral $\mathrm{U}(1)_{R}$ rotations $(\mathbb{A})$ under which $\phi^{\mathrm{a}}$ and $K$ must transform as

$$
\delta \phi^{\mathrm{a}}=\Lambda_{\mathrm{D}} \chi^{\mathrm{a}}+\frac{2 i}{3} \Lambda_{\mathrm{A}} \chi^{\mathrm{a}}, \quad \delta K=\delta \phi^{\mathrm{a}} K_{\mathrm{a}}+\text { h.c. }=2 \Lambda_{\mathrm{D}} K .
$$

The chiral function $\chi^{\mathrm{a}}(\phi)$ describes a homothetic conformal Killing vector,

$$
\nabla_{\mathrm{b}} \chi^{\mathrm{a}}=\delta_{\mathrm{b}}^{\mathrm{a}}, \quad \nabla_{\overline{\mathrm{b}}} \chi^{\mathrm{a}}=0,
$$

where $\nabla_{\mathrm{a}}$ and $\nabla_{\overline{\mathrm{a}}}$ are the target space covariant derivatives. The Kähler potential is $K \equiv \chi^{\mathrm{a}} \chi^{\overline{\mathrm{b}}} g_{\mathrm{a} \overline{\mathrm{b}}}$ and describes a Kähler cone [28]. At the component level, the Lagrangian includes a contribution from the Ricci scalar of the form $\frac{1}{6} \mathcal{R} K$. Usually one prefers instead a canonically-normalized Einstein-Hilbert term, which can be achieved by imposing the dilatation gauge $K=-3$ and simultaneously fixing the $\mathrm{U}(1)_{R}$ symmetry. This eliminates two scalar fields from the sigma model and converts the Kähler cone of dimension $2 n$ into a Hodge-Kähler manifold of dimension $2(n-1)$.

In the $\mathcal{N}=2$ setting, a similar picture emerges. A sigma model coupled to $\mathcal{N}=2$ conformal supergravity must be a hyperkähler cone [29] (see [28, 30] for the rigid superconformal case and [31] for a discussion in general dimensions), which is a hyperkähler manifold possessing a homothetic conformal Killing vector and $\mathrm{SU}(2)_{R}$ isometries that rotate the complex structures; such spaces are also known as Swann bundles [32]. There exists a one-to-one correspondence between $4 n$-dimensional hyperkähler cones and $4(n-1)$ dimensional quaternion-Kähler manifolds [32] (see also [33] as well as [34, 35] for recent discussions and references). The component action for a hyperkähler cone coupled to conformal supergravity was given in [29], where its relation to the Poincaré supergravitycoupled quaternion-Kähler action of [3] was also discussed: the elimination of four scalars in the target space comes from fixing the dilatation and $\mathrm{SU}(2)_{R}$ gauges.

Our goal in this paper is to reproduce in a systematic way the component action of [29] for a hyperkähler cone coupled to conformal supergravity directly from (curved) projective superspace. ${ }^{5}$ This construction is in principle sufficient because the one-to-one

\footnotetext{
${ }^{5}$ The corresponding actions in harmonic superspace were discussed in [36]. The relation between the unconstrained harmonic potentials and general quaternion-Kähler geometry was established in [37]. The derivation of the bosonic Lagrangian from harmonic superspace appeared in [38].
} 
correspondence between hyperkähler cones and quaternion-Kähler manifolds guarantees one may construct the general action for supergravity coupled to a quaternionic-Kähler sigma model by gauge-fixing the superconformal case appropriately [29, 33, 34]. We will return to the question of a native superspace approach in the conclusion; until then, we will always be referring to conformal supergravity when we mention locally supersymmetric sigma models. To accomplish our goal, we require a covariant approach to supergravitymatter systems employing projective superspace. Such an approach was developed in 4D by Kuzenko, Lindström, Roček, and Tartaglino-Mazzucchelli [19-22] using conventional $N=2$ superspace (in turn based on the work of Kuzenko and Tartaglino-Mazzucchelli in 5D [39-41]). This approach was later extended in [18], which clarified a number of issues and changed the superspace geometry to conformal superspace [42], which has a close relationship with the superconformal tensor calculus. For this paper, we will employ the conventions and projective superspace geometry of [18], but one could also employ those of [19-22]. The hypermultiplet action is given in curved superspace by

$$
-\frac{1}{2 \pi} \oint_{\mathcal{C}} \mathrm{d} \tau \int \mathrm{d}^{4} x \mathrm{~d}^{4} \theta^{+} \mathcal{E}^{--} \mathcal{F}^{++}\left(\Upsilon^{+}, \breve{\Upsilon}^{+}\right)
$$

We explicitly parametrize the $\mathrm{SU}(2)$ contour $\mathcal{C}$ by the coordinate $\tau$, and the measure $\mathcal{E}^{--}$is a superdeterminant of the relevant superspace vielbein. ${ }^{6}$ The generating function $\mathcal{F}^{++}$possesses no explicit dependence on $v^{i+}$ : this generalizes to curved superspace the superconformal version of (1.5), constructed originally in flat space [43] and ensures that the target space describes a hyperkähler cone [44]. The major barrier to this calculation is that the usual method of eliminating the infinite number of auxiliary fields in the flat superspace action (1.5) depends on introducing an intermediate $\mathcal{N}=1$ superspace. This is easy to do in flat superspace and has been accomplished recently in AdS superspace [45-48], but it is quite daunting in a general curved geometry. ${ }^{7}$ Instead, we will take inspiration from rigid harmonic superspace and proceed directly from $\mathcal{N}=2$ superspace to the component action. The elimination of the auxiliary fields will seem rather different at first glance from the $\mathcal{N}=1$ approach, but will actually involve solving the same set of equations; this implies that the coupling to conformal supergravity will in no way affect the elimination of the hypermultiplet auxiliaries. ${ }^{8}$ Along the way, we will derive explicit formulae in projective superspace for all of the geometric quantities necessary for describing the hyperkähler cone and its sigma model.

This paper is laid out as follows. Section 2 provides a review of how hyperkähler geometry can be derived from flat projective superspace via the $\mathcal{N}=1$ superspace method. Although this method seems to be useful mainly for rigid supersymmetric spaces such as Minkowski or AdS, many of the same formulae and notation will reoccur in later sections,

\footnotetext{
${ }^{6}$ In the flat space limit, $\mathcal{E}^{--}=v_{i}^{+} \mathrm{d} v^{i+} / \mathrm{d} \tau$ so that $\mathrm{d} \tau \mathcal{E}^{--}$reduces to the flat measure $v_{i}^{+} \mathrm{d} v^{i+}$. Further details can be found in [18].

${ }^{7}$ The approach of reducing curved $\mathcal{N}=2$ superfields to $\mathcal{N}=1$ superfields has been discussed in [49-53], but its application in this case would seem to be very difficult.

${ }^{8}$ In the quaternion-Kähler case, this is more subtle. As is evident from the corresponding harmonic [37] and projective [35] descriptions, a hypermultiplet compensator plays the role of the effective auxiliary variable for the on-shell hypermultiplet superfields.
} 
so some familiarity will be necessary. In section 3 , we describe how the full $\mathcal{N}=2$ superfield equations of motion lead to on-shell $\mathcal{N}=2$ hypermultiplets whose target space is a hyperkähler cone, and we derive all the geometric data we will need from projective superspace. We then describe in section 4 how to restrict the $\mathcal{N}=2$ equations of motion to only the auxiliary sector, eliminating the infinite tail of auxiliary fields while keeping the physical fields off-shell. In section 5 we test this approach by deriving the component action in the rigid supersymmetric case, before addressing the curved case in section 6 . We comment on our results and speculate about some open questions in the conclusion.

Two appendices are included. The first gives our conventions for vector multiplets and their component fields (these conventions were absent in [18]). The second provides some technical details necessary to calculate the final component action.

\section{A review of hyperkähler geometry from flat projective superspace}

Let us begin by reviewing how projective superspace permits the construction of hyperkähler sigma models in flat space. This material is well-known and we refer to the lecture notes [54] as well as [24] and [23, 55] for further details and the relevant references.

We begin with the flat projective superspace Lagrangian $\mathcal{F}^{++}$

$$
\mathcal{F}^{++}=\mathcal{F}^{++}\left(\Upsilon^{+}, \breve{\Upsilon}^{+}, v^{i+}\right)
$$

depending on arctic multiplets $\Upsilon^{I+}$, antarctic multiplets $\breve{\Upsilon}^{\bar{I}+}$ and the coordinate $v^{i+}$. It is analytic and holomorphic, $D_{\underline{\alpha}}^{+} \mathcal{F}^{++}=D^{++} \mathcal{F}^{++}=0$, by construction. The action is

$$
S=-\frac{1}{2 \pi} \oint_{\mathcal{C}} v_{i}^{+} \mathrm{d} v^{i+} \int \mathrm{d}^{4} x \mathrm{~d}^{4} \theta^{+} \mathcal{F}^{++}=-\frac{1}{2 \pi} \oint_{\mathcal{C}} v_{i}^{+} \mathrm{d} v^{i+} \int \mathrm{d}^{4} x\left(D^{-}\right)^{4} \mathcal{F}^{++} .
$$

This can be evaluated as an integral in $\mathcal{N}=1$ superspace. To do this, recast all superfields so that they depend solely on the complex coordinate $\zeta=v^{\underline{2}+} / v^{\underline{1}}+$ rather than $v^{\underline{1}}+$ and $v^{\underline{2}+}$ separately. For example, one introduces a new arctic superfield $\Upsilon^{I}(\zeta)$,

$$
\Upsilon^{I}:=\frac{1}{v^{1}+} \Upsilon^{I+}=\Phi^{I}+\zeta \Sigma^{I}+\sum_{n=2}^{\infty} \zeta^{n} \Upsilon_{n}^{I}
$$

If we interpret the components in this expansion as $\mathcal{N}=1$ superfields, then $\Phi^{I}$ is chiral and $\Sigma^{I}$ is complex linear, while the infinite tail of superfields $\Upsilon_{n}^{I}$ are unconstrained $\mathcal{N}=1$ superfields. The antarctic superfield possesses a similar expansion

$$
\breve{\Upsilon}^{\bar{I}}=\frac{1}{v^{2}+} \breve{\Upsilon}^{I+}=\bar{\Phi}^{\bar{I}}-\frac{1}{\zeta} \bar{\Sigma}^{\bar{I}}+\sum_{n=2}^{\infty}(-1)^{n} \zeta^{-n} \bar{\Upsilon}_{n}^{\bar{I}}
$$

Rewriting the projective superspace Lagrangian as $\mathcal{F}^{++}=i v^{\underline{1}+} v^{\underline{2}+} \mathcal{F}(\zeta)$, we find

$$
S=\int \mathrm{d}^{4} x \mathrm{~d}^{2} \theta_{\underline{1}} \mathrm{~d}^{2} \bar{\theta}^{\underline{1}} \mathscr{L}, \quad \mathscr{L}=\oint_{\mathcal{C}} \frac{\mathrm{d} \zeta}{2 \pi i \zeta} \mathcal{F}(\Upsilon, \breve{\Upsilon}, \zeta)
$$


in terms of the $\mathcal{N}=1$ superspace Lagrangian $\mathscr{L}$. Note that while $\mathcal{F}^{++}$had to be homogeneous of weight two in its parameters, no such constraint is imposed on $\mathcal{F}$.

Because the superfields $\Upsilon_{n}^{I}$ with $n \geq 2$ are unconstrained $\mathcal{N}=1$ superfields, their equations of motion are purely algebraic ${ }^{9}$

$$
\oint_{\mathcal{C}} \frac{\mathrm{d} \zeta}{2 \pi i \zeta} \frac{\partial \mathcal{F}}{\partial \Upsilon^{I}} \zeta^{n}=0, \quad \oint_{\mathcal{C}} \frac{\mathrm{d} \zeta}{2 \pi i \zeta} \frac{\partial \mathcal{F}}{\partial \breve{\Upsilon}^{\bar{I}}} \zeta^{-n}=0, \quad n \geq 2
$$

Imposing these, the Lagrangian becomes solely a function of $\Phi^{I}, \Sigma^{I}$ and their complex conjugates. Now perform a duality transformation, exchanging the complex linear superfield $\Sigma^{I}$ for a chiral superfield $\Psi_{I}$,

$$
S=\int \mathrm{d}^{4} x \mathrm{~d}^{2} \theta_{\underline{1}} \mathrm{~d}^{2} \bar{\theta}^{\underline{1}}\left(\mathscr{L}-\Sigma^{I} \Psi_{I}-\bar{\Sigma}^{I} \bar{\Psi}_{I}\right) .
$$

The equation of motion for $\Psi_{I}$ enforces the complex linearity of $\Sigma^{I}$, recovering (2.5). Alternatively, we can eliminate $\Sigma^{I}$ using its own equation of motion, effecting a Legendre transformation

$$
K(\Phi, \bar{\Phi}, \Psi, \bar{\Psi})=\mathscr{L}(\Phi, \bar{\Phi}, \Sigma, \bar{\Sigma})-\Sigma^{I} \Psi_{I}-\bar{\Sigma}^{I} \bar{\Psi}_{I}
$$

The resulting function $K$ is a Kähler potential with complex coordinates $\phi^{\mathrm{a}}=\left(\Phi^{I}, \Psi_{I}\right)$.

This Kähler potential describes a hyperkähler manifold. In addition to the manifest $\mathcal{N}=1$ supersymmetry, there is a hidden second supersymmetry on-shell, which manifests in $\mathcal{N}=1$ language as [59]

$$
\delta \phi^{\mathrm{a}}=\omega^{\mathrm{ab}} \bar{\rho}_{\dot{\alpha}} \bar{D}^{\dot{\alpha}} K_{\mathrm{b}}=\omega^{\mathrm{a}} \overline{\mathrm{b}} \bar{\rho}_{\dot{\alpha}} \bar{D}^{\dot{\alpha}} \bar{\phi}^{\overline{\mathrm{b}}}
$$

for constant $\bar{\rho}_{\dot{\alpha}} \equiv \bar{\xi}_{\dot{\alpha}} \underline{2}$, the second supersymmetry parameter. The tensor $\omega^{\mathrm{ab}}$ is antisymmetric, covariantly constant, and obeys $\omega^{\mathrm{a}}{ }_{\mathrm{b}} \omega^{\bar{b}}{ }_{\mathrm{c}}=\omega^{\mathrm{ab}} \omega_{\mathrm{bc}}=-\delta^{\mathrm{a}}{ }_{\mathrm{c}}$. The special coordinates $\phi^{\mathrm{a}}=\left(\Phi^{I}, \Psi_{I}\right)$ are Darboux coordinates for which $[24,55]$

$$
\omega^{\mathrm{ab}}=\left(\begin{array}{cc}
0 & \delta^{I} J \\
-\delta_{I}{ }^{J} & 0
\end{array}\right), \quad \omega_{\mathrm{ab}}=\left(\begin{array}{cc}
0 & \delta_{I}^{J} \\
-\delta^{I}{ }_{J} & 0
\end{array}\right) .
$$

The presence of such an antisymmetric covariantly constant tensor ensures that the Kähler manifold is actually hyperkähler, with a triplet of closed hyperkähler two-forms $\Omega_{i j}$,

$$
\begin{aligned}
& \Omega_{\underline{11}}=\frac{1}{2} \omega_{\mathrm{ab}} \mathrm{d} \phi^{\mathrm{a}} \wedge \mathrm{d} \phi^{\mathrm{b}}=\mathrm{d} \Phi^{I} \wedge \mathrm{d} \Psi_{I}, \\
& \Omega_{\underline{12}}=\Omega_{\underline{21}}=\frac{1}{2} g_{\mathrm{a} \overline{\mathrm{b}}} \mathrm{d} \phi^{\mathrm{a}} \wedge \mathrm{d} \bar{\phi}^{\overline{\mathrm{b}}}, \\
& \Omega_{\underline{22}}=\frac{1}{2} \bar{\omega}_{\overline{\mathrm{a}} \overline{\mathrm{b}}} \mathrm{d} \bar{\phi}^{\overline{\mathrm{a}}} \wedge \mathrm{d} \bar{\phi}^{\overline{\mathrm{b}}}=\mathrm{d} \bar{\Phi}^{\bar{I}} \wedge \mathrm{d} \bar{\Psi}_{I} .
\end{aligned}
$$

\footnotetext{
${ }^{9}$ These equations of motion were described in [13]. They were given explicitly in [56, 57], for a class of $\zeta$-independent functions $\mathcal{F}$ whose resulting hyperkähler manifolds were shown to be cotangent bundles of Kähler manifolds, building off a related observation in [58]. The full explicit form, discussed here, appeared later in [24].
} 
The three hyperkähler two-forms are related to three covariantly constant complex structures $\left(\mathcal{J}_{i j}\right)^{\mu}{ }_{\nu}=g^{\mu \rho}\left(\Omega_{i j}\right)_{\rho \nu}$,

$$
\left(\mathcal{J}_{\underline{11}}\right)^{\mu}{ }_{\nu}=\left(\begin{array}{cc}
0 & 0 \\
\omega^{\overline{\mathrm{a}}} & 0
\end{array}\right), \quad\left(\mathcal{J}_{\underline{12}}\right)^{\mu}{ }_{\nu}=\left(\begin{array}{cc}
-\frac{1}{2} \delta^{\mathrm{a}}{ }_{\mathrm{b}} & 0 \\
0 & \frac{1}{2} \delta^{\overline{\mathrm{a}}_{\overline{\mathrm{b}}}}
\end{array}\right), \quad\left(\mathcal{J}_{\underline{22}}\right)^{\mu}{ }_{\nu}=\left(\begin{array}{cc}
0 & \omega^{\mathrm{a}} \overline{\mathrm{b}} \\
0 & 0
\end{array}\right),
$$

which obey the multiplication rule

$$
\mathcal{J}_{i j} \mathcal{J}_{k l}=\frac{1}{2} \epsilon_{i(k} \epsilon_{l) j}+\frac{1}{2}\left(\epsilon_{i(k} \mathcal{J}_{l) j}+\epsilon_{j(k} \mathcal{J}_{l) i}\right) .
$$

Introducing $\mathcal{J}_{A}:=-i\left(\tau_{A}\right)^{i}{ }_{j} \mathcal{J}^{j}{ }_{i}, A=1,2,3$ with the Pauli matrices $\left(\tau_{A}\right)^{i}{ }_{j}$, the multiplication rule becomes that of the quaternions, $\mathcal{J}_{A} \mathcal{J}_{B}=-\delta_{A B}+\epsilon_{A B C} \mathcal{J}_{C}$. The complex structures in this form are given by

$$
\left(\mathcal{J}_{1}\right)^{\mu}{ }_{\nu}=\left(\begin{array}{cc}
0 & -i \omega^{\mathrm{a}} \overline{\mathrm{b}} \\
i \omega^{\overline{\mathrm{a}}}{ }_{\mathrm{b}} & 0
\end{array}\right), \quad\left(\mathcal{J}_{2}\right)^{\mu}{ }_{\nu}=\left(\begin{array}{cc}
0 & \omega^{\mathrm{a}} \overline{\mathrm{b}} \\
\omega^{\overline{\mathrm{a}}} & 0
\end{array}\right), \quad\left(\mathcal{J}_{3}\right)^{\mu}{ }_{\nu}=\left(\begin{array}{cc}
i \delta^{\mathrm{a}}{ }_{\mathrm{b}} & 0 \\
0 & -i \delta^{\overline{\mathrm{a}}_{\overline{\mathrm{b}}}}
\end{array}\right) .
$$

$\mathcal{J}_{3}=-2 i \mathcal{J}_{12}$ is the complex structure associated with the manifest $\mathcal{N}=1$ supersymmetry.

We will eventually be interested in the case where the model is superconformal [44]. This amounts to the condition that $\mathcal{F}^{++}$is homogeneous of degree two, $2 \mathcal{F}^{++}=\mathcal{F}_{I}^{+} \Upsilon^{I+}+$ $\mathcal{F}_{\bar{I}}^{+} \breve{\Upsilon}^{\bar{I}+}$, which is equivalent to requiring the projective Lagrangian $\mathcal{F}^{++}$to be independent of $v^{i+}$. In contrast to the $\mathcal{N}=1$ situation, there is no requirement that $\mathcal{F}^{++}$be separately homogeneous in $\Upsilon^{I+}$. This is because arctic and antarctic multiplets are both inert under $\mathrm{U}(1)_{R}$, so there is no superconformal symmetry that distinguishes between them. ${ }^{10}$

Now the Kähler potential $K$ turns out to possess a chiral homothetic conformal Killing vector $(\mathrm{CKV}) \chi^{\mathrm{a}}$ obeying (1.8), implying that the Kähler potential can be chosen (up to a Kähler transformation) as $K=\chi^{\mathrm{a}} \chi_{\mathrm{a}}$. For the Darboux coordinate system, the homothetic conformal Killing vector takes the simple form $\chi^{\mathrm{a}}=\left(\Phi^{I}, \Psi_{I}\right)$. The presence of $\chi^{\mathrm{a}}$ ensures that the hyperkähler manifold is actually a hyperkähler cone. In addition to the two supersymmetry transformations, it admits a full set of $\mathcal{N}=2$ superconformal transformations, including dilatation and $\mathrm{SU}(2)_{R}$ transformations. These manifest as [55]

$$
\delta \phi^{\mathrm{a}}=\Lambda_{\mathrm{D}} \chi^{\mathrm{a}}+\lambda^{\underline{12}} \chi^{\mathrm{a}}-\lambda^{\underline{22}} \omega^{\mathrm{ab}} \chi_{\mathrm{b}},
$$

where $\Lambda_{\mathrm{D}}$ is the scale parameter and $\lambda^{i}{ }_{j}$ is the $\mathrm{SU}(2)_{R}$ transformation parameter. The fields $\phi^{\mathrm{a}}$ are inert under $\mathrm{U}(1)_{R}$.

\section{Hyperkähler geometry and on-shell $\mathcal{N}=2$ superfields}

Our goal in this section is to establish the geometric properties of the target space geometry of (1.9) (including the results of the previous section) without explicitly reducing to $\mathcal{N}=1$ superspace. This is necessary in order to derive the component action in the presence of

\footnotetext{
${ }^{10}$ Moreover, imposing a separate homogeneity condition for the arctics and antarctics is equivalent to assigning an additional global U(1) isometry to the projective Lagrangian, which descends to the hyperkähler manifold as a new triholomorphic isometry. Such an isometry is not generically present in hyperkähler cones. This issue was already noted in the context of $3 D$ sigma models with $(3,0)$ AdS supersymmetry [48].
} 
supergravity, where an $\mathcal{N}=1$ superspace is not readily available. Our goal will be to reduce the arctic superfields to on-shell $\mathcal{N}=2$ superfields: we will define these as $\phi^{\mathrm{a}}=\left(\Phi^{I}, \Psi_{I}\right)$ in analogy to the $\mathcal{N}=1$ superfields of the previous section.

Our starting point is equivalent to that discussed in [57] and [24]: we will analyze the full $\mathcal{N}=2$ superfield equations of motion. If the action (1.9) is stationary under arbitrary variations of $\Upsilon^{I+}$,

$$
-\frac{1}{2 \pi} \oint_{\mathcal{C}} \mathrm{d} \tau \int \mathrm{d}^{4} x \mathrm{~d}^{4} \theta^{+} \mathcal{E}^{--} \delta \Upsilon^{I+} \frac{\partial \mathcal{F}^{++}}{\partial \Upsilon^{I+}}=0
$$

then $\partial \mathcal{F}^{++} / \partial \Upsilon^{I+}$ must itself be an arctic superfield. (The integral vanishes in this case since the contour $\mathcal{C}$ can be retracted to the north pole without encountering singularities.) This result holds both in the rigid and locally supersymmetric situations. This leads one to introduce superfields $\Gamma_{I}^{+}$and $\breve{\Gamma}_{\bar{I}}^{+}$, defined by

$$
\Gamma_{I}^{+}:=-i \frac{\partial \mathcal{F}^{++}}{\partial \Upsilon^{I+}}, \quad \breve{\Gamma}_{\bar{I}}^{+}:=i \frac{\partial \mathcal{F}^{++}}{\partial \breve{\Upsilon}^{\bar{I}+}} .
$$

The equations of motion require $\Gamma_{I}^{+}$and $\breve{\Gamma}_{\bar{I}}^{+}$to be, respectively, arctic and antarctic. ${ }^{11}$ The superfields $\Phi^{I}$ and $\Psi_{I}$ correspond to the leading terms in the $\zeta$ expansions of $\Upsilon^{I+}$ and $\Gamma_{I}^{+}$,

$$
\Upsilon^{I+}=v^{\underline{1}+}\left(\Phi^{I}+\mathcal{O}(\zeta)\right), \quad \Gamma_{I}^{+}=v^{\underline{1}+}\left(\Psi_{I}+\mathcal{O}(\zeta)\right)
$$

and can be defined equivalently via contour integration, ${ }^{12}$

$$
\Phi^{I}=\oint_{\mathcal{C}} \frac{\mathrm{d} \zeta}{2 \pi i \zeta} \frac{\Upsilon^{I+}}{v^{\underline{1}+}}, \quad \Psi_{I}=\oint_{\mathcal{C}} \frac{\mathrm{d} \zeta}{2 \pi i \zeta} \frac{\Gamma_{I}^{+}}{v \underline{1}+} .
$$

We will assume that the contour $\mathcal{C}$ winds around the north pole (and thus the south pole as well) exactly once and that the arctic (antarctic) multiplets possess no singularities in the northern (southern) chart bounded by $\mathcal{C}$. Consistency with the flat space $\mathcal{N}=1$ analysis implies that the on-shell $\mathcal{N}=2$ superfields $\Upsilon^{I+}$ and $\breve{\Upsilon}^{\bar{I}+}$ must be given by power series in the $\mathcal{N}=2$ superfields $\phi^{\mathrm{a}}=\left(\Phi^{I}, \Psi_{I}\right)$ and their complex conjugates. Because no fields of the conformal supergravity multiplet appear in the solution of the power series, the sigma model for local supersymmetry will be identical as for the rigid superconformal case.

The $\mathcal{N}=2$ superconformal transformations of $\Phi^{I}$ and $\Psi_{I}$ can be derived from their definitions (3.4),

$$
\delta \Phi^{I}=\oint_{\mathcal{C}} \frac{\mathrm{d} \zeta}{2 \pi i \zeta} \frac{\delta \Upsilon^{I+}}{v \underline{\underline{1}+}}, \quad \delta \Psi_{I}=\oint_{\mathcal{C}} \frac{\mathrm{d} \zeta}{2 \pi i \zeta} \frac{\delta \Gamma_{I}^{+}}{v \underline{1}+}
$$

where $\delta$ consists of any local (super)symmetry transformation. Consistency dictates that $\delta \Upsilon^{I+}$ can equivalently be calculated by

$$
\delta \Upsilon^{I+}=\delta \phi^{\mathrm{a}} \partial_{\mathrm{a}} \Upsilon^{I+}+\delta \bar{\phi}^{\overline{\mathrm{a}}} \partial_{\overline{\mathrm{a}}} \Upsilon^{I+}
$$

Using only these results, let us briefly discuss how the geometry of the target space follows.

\footnotetext{
${ }^{11}$ These can be interpreted as dual superfields; see [60] for a discussion of polar-polar duality.

${ }^{12}$ In defining the $\mathcal{N}=2$ superfields $\Phi^{I}$ and $\Psi_{I}$, we implicitly choose to work in the central gauge of projective superspace as discussed in [18].
} 


\subsection{Hyperkähler geometry}

Here we follow closely the approach of [24]. The two-form ${ }^{13}$

$$
\Omega^{++}=\mathrm{d} \Upsilon^{I+} \wedge \mathrm{d} \Gamma_{I}^{+}=\mathrm{d} \breve{\Upsilon}^{\bar{I}+} \wedge \mathrm{d} \breve{\Gamma}_{\bar{I}}^{+}
$$

is both arctic and antarctic when the full $\mathcal{N}=2$ equations of motion are imposed, so it must be globally-defined on the auxiliary manifold. This means it is given by $\Omega^{++}=\Omega_{i j} v^{i+} v^{j+}$ for a triplet of two-forms $\Omega_{i j}$. It is obvious from its definition that $\Omega_{11}=\mathrm{d} \Phi^{I} \wedge \mathrm{d} \Psi_{I}$ and $\Omega_{\underline{22}}=\mathrm{d} \bar{\Phi}^{\bar{I}} \wedge \mathrm{d} \Psi_{\bar{I}}$, while $\Omega_{\underline{12}}$ can only be a $(1,1)$ form, $\Omega_{\underline{12}}=\frac{1}{2} \mathrm{~d} \phi^{\mathrm{a}} \wedge \mathrm{d} \bar{\phi}^{\overline{\mathrm{b}}} g_{\mathrm{a} \overline{\mathrm{b}}}$, for some tensor $g_{\mathrm{a} \overline{\mathrm{b}}}$. The expression for $\Omega^{++}$can then be written as

$$
\Omega^{++}=v^{\underline{1}+} v^{\underline{2}}+\left(\frac{1}{2 \zeta} \mathrm{d} \phi^{\mathrm{a}} \wedge \mathrm{d} \phi^{\mathrm{b}} \omega_{\mathrm{ab}}+\mathrm{d} \phi^{\mathrm{a}} \wedge \mathrm{d} \bar{\phi}^{\overline{\mathrm{b}}} g_{\mathrm{a} \overline{\mathrm{b}}}+\frac{\zeta}{2} \mathrm{~d} \bar{\phi}^{\overline{\mathrm{a}}} \wedge \mathrm{d} \bar{\phi}^{\overline{\mathrm{b}}} \omega_{\overline{\mathrm{a}} \overline{\mathrm{b}}}\right)
$$

identifying

$$
\begin{aligned}
& \omega_{\mathrm{ab}}=\frac{1}{v^{\underline{1}+} v \underline{1}+}\left(\partial_{\mathrm{a}} \Upsilon^{I+} \partial_{\mathrm{b}} \Gamma_{I}^{+}-\partial_{\mathrm{a}} \Gamma_{I}^{+} \partial_{\mathrm{b}} \Upsilon^{I+}\right), \\
& g_{\mathrm{a} \overline{\mathrm{b}}}=\frac{1}{v^{\underline{1}+} v^{\underline{2}}+}\left(\partial_{\mathrm{a}} \Upsilon^{I+} \partial_{\overline{\mathrm{b}}} \Gamma_{I}^{+}-\partial_{\mathrm{a}} \Gamma_{I}^{+} \partial_{\overline{\mathrm{b}}} \Upsilon^{I+}\right), \\
& \omega_{\overline{\mathrm{a}} \overline{\mathrm{b}}}=\frac{1}{v^{\underline{2}+} v^{\underline{2}}+}\left(\partial_{\overline{\mathrm{a}}} \Upsilon^{I+} \partial_{\overline{\mathrm{b}}} \Gamma_{I}^{+}-\partial_{\overline{\mathrm{a}}} \Gamma_{I}^{+} \partial_{\overline{\mathrm{b}}} \Upsilon^{I+}\right) .
\end{aligned}
$$

These relations also hold upon replacing $\Upsilon^{I+} \rightarrow \breve{\Upsilon} \breve{I}+$ and $\Gamma_{I}^{+} \rightarrow \breve{\Gamma}_{\bar{I}}^{+}$.

Because $\Omega^{++}$is closed, it follows that both $\omega_{\mathrm{ab}}$ and $g_{\mathrm{a} \overline{\mathrm{b}}}$ must be closed when viewed respectively as $(2,0)$ and $(1,1)$ forms. (The closure of $\omega_{\mathrm{ab}}$ is obvious in the Darboux coordinates.) The closure of $g_{\mathrm{ab}}$ implies that it must be the second derivative of a function $K$. This function can be chosen as in the explicit $\mathcal{N}=1$ reduction as

$$
K=\mathscr{L}-\Sigma^{I} \Psi_{I}-\bar{\Sigma}^{\bar{I}} \bar{\Psi}_{\bar{I}}, \quad \mathscr{L}:=\oint_{\mathcal{C}} \frac{\mathrm{d} \zeta}{2 \pi i \zeta} \frac{\mathcal{F}^{++}}{i v \underline{1}^{+} v^{+}} .
$$

Identifying $\Sigma^{I}$ and $\Sigma_{I}$ as the second terms in the expansions of $\Upsilon^{I+}$ and $\Gamma_{I}^{+}$, one can show

$$
\begin{aligned}
\partial_{\mathrm{a}} K & =\Sigma_{I} \partial_{\mathrm{a}} \Phi^{I}-\Sigma^{I} \partial_{\mathrm{a}} \Psi_{I}, \\
\partial_{\overline{\mathrm{b}}} \partial_{\mathrm{a}} K & =\partial_{\overline{\mathrm{b}}} \Sigma_{I} \partial_{\mathrm{a}} \Phi^{I}-\partial_{\overline{\mathrm{b}}} \Sigma^{I} \partial_{\mathrm{a}} \Psi_{I}=\oint_{\mathcal{C}} \frac{\mathrm{d} \zeta}{2 \pi i \zeta} \frac{1}{\zeta}\left(\partial_{\mathrm{a}} \Upsilon^{I} \partial_{\overline{\mathrm{b}}} \Gamma_{I}-\partial_{\mathrm{a}} \Gamma_{I} \partial_{\overline{\mathrm{b}}} \Upsilon^{I}\right) \equiv g_{\mathrm{a} \overline{\mathrm{b}}} .
\end{aligned}
$$

Let us next establish that $\omega_{\mathrm{ab}}=g_{\mathrm{a} \overline{\mathrm{c}}} \omega^{\overline{\mathrm{c}} \overline{\mathrm{d}}} g_{\overline{\mathrm{d}} \mathrm{b}}$ where $\omega^{\overline{\mathrm{a}} \overline{\mathrm{b}}}$ is the inverse of $\omega_{\overline{\mathrm{a}} \overline{\mathrm{b}}}$, given by

$$
\omega^{\overline{\mathrm{a}} \overline{\mathrm{b}}}=\left(\begin{array}{cc}
0 & \delta^{\bar{I}} \bar{J} \\
-\delta_{\bar{I}}^{\bar{J}} & 0
\end{array}\right), \quad \omega^{\overline{\mathrm{a}} \overline{\mathrm{b}}} \omega_{\overline{\mathrm{b}} \overline{\mathrm{c}}}=-\delta^{\overline{\mathrm{a}}} \overline{\mathrm{c}} .
$$

A proof of this follows by using the explicit antarctic expression for $g_{\mathrm{a} \overline{\mathrm{b}}}$ and writing

$$
\begin{aligned}
g_{\mathrm{a} \overline{\mathrm{c}}} \omega^{\overline{\mathrm{c}}} g_{\overline{\mathrm{db}} \mathrm{b}}= & -\frac{1}{\left(v^{\underline{1}+} v^{\underline{2}+}\right)^{2}}\left(\partial_{\mathrm{a}} \breve{\Upsilon}^{\bar{I}+} \partial_{\bar{K}} \breve{\Gamma}_{\bar{I}}^{+}-\partial_{\mathrm{a}} \breve{\Gamma}_{\bar{I}}^{+} \partial_{\bar{K}} \breve{\Upsilon}^{\bar{I}+}\right)\left(\partial^{\bar{K}} \breve{\Upsilon}^{\bar{J}+} \partial_{\mathrm{b}} \breve{\Gamma}_{\bar{J}}^{+}-\partial^{\bar{K}} \breve{\Gamma}_{\bar{J}}^{+} \partial_{\mathrm{b}} \breve{\Upsilon}^{\bar{J}+}\right) \\
& +\frac{1}{\left(v^{\underline{1}+} v^{2}+\right)^{2}}\left(\partial_{\mathrm{a}} \breve{\Upsilon}^{\bar{I}+} \partial^{\bar{K}} \breve{\Gamma}_{\bar{I}}^{+}-\partial_{\mathrm{a}} \breve{\Gamma}_{\bar{I}}^{+} \partial^{\bar{K}} \breve{\Upsilon}^{\bar{I}+}\right)\left(\partial_{\bar{K}} \breve{\Upsilon}^{\bar{J}+} \partial_{\mathrm{b}} \breve{\Gamma}_{\bar{J}}^{+}-\partial_{\bar{K}} \breve{\Gamma}_{\bar{J}}^{+} \partial_{\mathrm{b}} \breve{\Upsilon}^{\bar{J}+}\right) \\
\partial_{\bar{I}}:= & \frac{\partial}{\partial \bar{\Phi}^{\bar{I}}}, \quad \partial^{\bar{I}}=\frac{\partial}{\partial \bar{\Psi}_{\bar{I}}} .
\end{aligned}
$$

\footnotetext{
${ }^{13}$ For target space quantities such as $\Omega^{++}$, we follow the standard conventions for differential forms rather than the superspace conventions of e.g. [61].
} 
This expression must be independent of $v^{i+}$, so we can discard all terms that go as negative powers of $\zeta$. Using

$$
\begin{aligned}
& \partial_{\bar{J}} \breve{\Upsilon}^{\bar{I}+}=v^{2+}\left(\delta_{\bar{J}}^{\bar{I}}+\mathcal{O}(1 / \zeta)\right), \quad \partial^{\bar{J}} \breve{\Gamma}_{\bar{I}}^{+}=v^{\underline{2}+}\left(\delta_{\bar{I}}^{\bar{J}}+\mathcal{O}(1 / \zeta)\right), \\
& \partial_{\mathrm{a}} \breve{\Upsilon}^{\bar{I}+} \sim \partial_{\mathrm{a}} \breve{\Gamma}^{\bar{I}+} \sim \partial^{\bar{J}} \breve{\Upsilon}^{\bar{I}+} \sim \partial^{\bar{J}} \breve{\Gamma}_{\bar{I}}^{+} \sim v^{\underline{2}+} \mathcal{O}(1 / \zeta),
\end{aligned}
$$

we see that the only terms that contribute are

$$
g_{\mathrm{a} \overline{\mathrm{c}}} \omega^{\overline{\mathrm{c}} \overline{\mathrm{d}}} g_{\overline{\mathrm{d} b}}=\frac{1}{(v \underline{\underline{1}+})^{2}}\left(\partial_{\mathrm{a}} \breve{\Upsilon}^{\bar{I}+} \partial_{\mathrm{b}} \breve{\Gamma}_{\bar{I}}^{+}-\partial_{\mathrm{a}} \breve{\Gamma}_{\bar{I}}^{+} \partial_{\mathrm{b}} \breve{\Upsilon}^{\bar{I}+}\right)=\omega_{\mathrm{ab}}
$$

which is what we wished to establish. This result actually guarantees that $g_{\mathrm{ab}}$ is invertible because both sides of (3.15) must have non-vanishing determinant. In other words, the non-degeneracy of the metric $g_{\mathrm{a}}$ is implied if we can solve the equations (3.1) completely in terms of the coordinates $\Phi^{I}$ and $\Psi_{I}$. This equality also guarantees that $\omega_{\mathrm{ab}}$ is covariantly constant. It follows that the manifold is hyperkähler.

\subsection{Gauged isometries from projective superspace}

Suppose that the projective superspace Lagrangian possesses some gauge invariance that is, the arctic multiplets possess gauged holomorphic isometries of the form

$$
\delta_{g} \Upsilon^{I+}=\lambda^{r} \mathcal{J}_{r}^{I+}, \quad \mathcal{J}_{r}^{I+}=\mathcal{J}_{r}^{I+}\left(\Upsilon^{+}, v^{i+}\right),
$$

for an arctic function $\mathcal{J}_{r}^{I+}$, and similarly for the antarctic multiplets, with $r$ labelling the adjoint representation of the gauge group. Let us show how these descend to triholomorphic isometries in the hyperkähler manifold, rederiving the results of [59]. ${ }^{14}$

Because the projective Lagrangian is gauge-invariant, ${ }^{15}$

$$
\delta_{g} \mathcal{F}^{++}=i \lambda^{r}\left(\Gamma_{I}^{+} \mathcal{J}_{r}^{I+}-\breve{\Gamma}_{\bar{I}}^{+} \breve{\mathcal{J}}_{r}^{\bar{I}+}\right)=0,
$$

we may introduce a real quantity

$$
D_{r}^{++}:=\Gamma_{I}^{+} \mathcal{J}_{r}^{I+}=\breve{\Gamma}_{\bar{I}}^{+} \breve{\mathcal{J}}_{r}^{\bar{I}+} .
$$

This is the $\mathcal{N}=2$ moment map (or Killing potential) in projective superspace. ${ }^{16}$ By construction, the gauge transformation of $\Gamma_{I}^{+}$is

$$
\delta_{g} \Gamma_{I}^{+} \equiv \lambda^{r} \mathcal{J}_{r I}^{+}=-\lambda^{r} \partial_{I+} \mathcal{J}_{r}^{J+} \Gamma_{J}^{+}, \quad \partial_{I+}:=\frac{\partial}{\partial \Upsilon^{I+}}
$$

\footnotetext{
${ }^{14} \mathrm{~A}$ treatment based on $\mathcal{N}=1$ superspace methods can be found in [62] and [63] for flat $4 \mathrm{D}$ and $5 \mathrm{D}$ cases, and $[47,48]$ for AdS geometries.

${ }^{15}$ It is actually not necessary for the Lagrangian to be fully gauge invariant, provided that one can consistently introduce a naked prepotential to counter its gauge transformation property. As discussed in [59], the prepotential can be absorbed in a covariant framework by introducing a fictitious multiplet that drops out of the action (its metric vanishes), except for its modification of the moment map. In this way, we retain the description above with only covariant hypermultiplets and no prepotentials. We thank Martin Roček for pointing out this important subtlety.

${ }^{16}$ The $\mathcal{N}=1$ formulation of the $\mathcal{N}=2$ Killing potential appeared in [59]. Harmonic and projective superspace formulations appeared explicitly in [36] and [63].
} 
and so it follows that $D_{r}^{++}$transforms in the co-adjoint of the gauge group,

$$
\delta_{g} D_{r}^{++}=\lambda^{s} \Gamma_{I}^{+}\left(\mathcal{J}_{s}^{J+} \partial_{J+} \mathcal{J}_{r}^{I+}-\mathcal{J}_{r}^{J+} \partial_{J+} \mathcal{J}_{s}^{I+}\right)=-\lambda^{s} f_{s r}{ }^{t} D_{t}^{++}
$$

When the equations of motion are imposed, the gauge transformations of the arctic fields must be manifested on the target space of complex fields. As a consequence of the transformation properties of $\Upsilon^{I+}$ and $\Gamma_{I}^{+}$, the two-form $\Omega^{++}$is gauge-invariant. Interpreting the gauge transformation as a target space transformation, it follows that $\mathcal{L}_{J} \Omega^{++}$vanishes, ensuring that gauged isometries in projective superspace descend to triholomorphic isometries in the target space. To derive this explicitly, observe that for $\mathcal{J}_{r}^{I+}\left(\Upsilon^{+}, v^{i+}\right) \equiv v^{\underline{1}+} \mathcal{J}_{r}^{I}(\Upsilon, \zeta)$,

$$
\delta_{g} \phi^{\mathrm{a}}=\lambda^{r} J_{r}^{\mathrm{a}}, \quad J_{r}^{I}=\mathcal{J}_{r}^{I}(\Phi, 0), \quad J_{r I}=-\partial_{I} J_{r}^{J}(\Phi) \Psi_{J}
$$

Now $D_{r}^{++}$must be both arctic and antarctic and so must be given by $D_{r i j} v^{i+} v^{j+}$, where

$$
\begin{aligned}
D_{r}^{++} & =v^{\underline{1}+} v^{\underline{2}+}\left(\frac{1}{\zeta} \Lambda_{r}+i D_{r}+\zeta \bar{\Lambda}_{r}\right), \\
\Lambda_{r} & =\Psi_{I} J_{r}^{I}(\Phi), \quad D_{r}=-i J_{r}^{\mathrm{a}} K_{\mathrm{a}}=i J_{r}^{\overline{\mathrm{a}}} K_{\overline{\mathrm{a}}} .
\end{aligned}
$$

The quantity $D_{r}$ is the $\mathcal{N}=1$ Killing potential and is related to the holomorphic quantity $\Lambda_{r}$ and the Killing vector $J_{r}^{\mathrm{a}}$ via [59]

$$
J_{r}^{\mathrm{a}}=i g^{\mathrm{a} \overline{\mathrm{b}}} \partial_{\overline{\mathrm{b}}} D_{r}=\omega^{\mathrm{ab}} \partial_{\mathrm{b}} \Lambda_{r}, \quad J_{r}^{\overline{\mathrm{a}}}=-i g^{\overline{\mathrm{a}} \mathrm{b}} \partial_{\mathrm{b}} D_{r}=\omega^{\overline{\mathrm{a}} \overline{\mathrm{b}}} \partial_{\overline{\mathrm{b}}} \bar{\Lambda}_{r}
$$

The relations (3.23) can equivalently be written

$$
\nabla_{\mu} D_{r}^{i j}=-\left(\Omega^{i j}\right)_{\mu \nu} J_{r}^{\nu}
$$

From this equation, one can prove that the Killing vector $J_{r}^{\mu}$ is triholomorphic.

It is worth mentioning that if $\mathcal{F}^{++}$is independent of $v^{i+}$, so that the target space is a hyperkähler cone, $\mathcal{J}_{r}^{I+}$ must also be independent of $v^{i+}$ and homogeneous in $\Upsilon^{I+}$ of degree one, $\Upsilon^{J+} \partial_{J+} \mathcal{J}_{r}^{I+}=\mathcal{J}_{r}^{I+}$. The $\mathcal{N}=2$ moment map can then equivalently be defined as

$$
D_{r}^{++}=\frac{1}{2} \Gamma_{I}^{+} X_{r} \Upsilon^{I+}-\frac{1}{2} \Upsilon^{I+} X_{r} \Gamma_{I}^{+}=\frac{1}{2} \Gamma_{I}^{+} \mathcal{J}_{r}^{I+}-\frac{1}{2} \Upsilon^{I+} \mathcal{J}_{r I}^{+}
$$

This can be derived from $\Omega^{++}$, replacing one $\mathrm{d}$ with $D^{0}$ and the other with the gauge generator $X_{r}$, leading to an explicit expression for its components,

$$
D_{r i j}=-\frac{1}{2}\left(\Omega_{i j}\right)_{\mu \nu} \chi^{\mu} J_{r}^{\nu} \quad \Longrightarrow \quad \Lambda_{r}=-\frac{1}{2} \omega_{\mathrm{ab}} \chi^{\mathrm{a}} J_{r}^{\mathrm{b}}, \quad D_{r}=-\frac{i}{2}\left(J_{r}^{\mathrm{a}} \chi_{\mathrm{a}}-J_{r}^{\overline{\mathrm{a}}} \chi_{\overline{\mathrm{a}}}\right),
$$

in terms of the homothetic conformal Killing vector $\chi^{\mu}$. 


\subsection{Superconformal isometries}

Now let us analyze the superconformal properties of the target space, following roughly the same approach as $[44,55]$, to which we refer for further details. The arctic multiplet $\Upsilon^{I+}$ transforms locally under dilatations and $\mathrm{SU}(2)_{R}$ transformations as

$$
\begin{aligned}
\delta \Upsilon^{I+} & =\Lambda_{\mathrm{D}} \mathbb{D} \Upsilon^{I+}+\lambda^{i}{ }_{j} I^{j}{ }_{i} \Upsilon^{I+}=\left(\Lambda_{\mathrm{D}}+\lambda^{-+}\right) \Upsilon^{I+}-\lambda^{++} D^{--} \Upsilon^{I+}, \\
\lambda^{++} & =\lambda^{i j} v_{i}^{+} v_{j}^{+}, \quad \lambda^{+-}=\lambda^{i j} v_{i}^{+} v_{j}^{-},
\end{aligned}
$$

and similarly for $\breve{\Upsilon}^{\bar{I}+}$. Both are inert under $\mathrm{U}(1)_{R}$, and so are their dual fields $\Gamma_{I}^{+}$and $\breve{\Gamma}_{\bar{I}}^{+}$. The above transformation should map to the target space as

$$
\delta \phi^{\mathrm{a}}=\Lambda_{\mathrm{D}} k_{\mathrm{D}}^{\mathrm{a}}+\lambda^{i}{ }_{j} k^{j}{ }_{i}^{\mathrm{a}}=\Lambda_{\mathrm{D}} k_{\mathrm{D}}^{\mathrm{a}}-\lambda^{i j} k_{i j}{ }^{\mathrm{a}}
$$

for some choice of vectors $k_{\mathrm{D}}^{\mathrm{a}}:=\mathbb{D} \phi^{\mathrm{a}}$ and $k_{i j}{ }^{\mathrm{a}}:=I_{i j} \phi^{\mathrm{a}}$. Let us recover their properties using projective superspace.

Superconformal invariance dictates that the projective Lagrangian transforms as

$$
\delta \mathcal{F}^{++}=\left(2 \Lambda_{\mathrm{D}}+2 \lambda^{+-}\right) \mathcal{F}^{++}-\lambda^{++} D^{--} \mathcal{F}^{++},
$$

implying that $\mathcal{F}^{++}$is homogeneous of degree two in the projective multiplets and possesses no explicit dependence on $v^{i+}$. It follows that the fields $\Gamma_{I}^{+}$and $\breve{\Gamma}_{\bar{I}}^{+}$transform in the same way as $\Upsilon^{I+}$ and $\breve{\Upsilon}{ }^{\bar{I}+}$ under superconformal transformations. From the definitions (3.4) of $\Phi^{I}$ and $\Psi_{I}$, it is clear that they possess unit dilatation weight, so we establish $k_{\mathrm{D}}^{\mathrm{a}}:=\mathbb{D} \phi^{\mathrm{a}}=$ $\left(\Phi^{I}, \Psi_{I}\right)$. A similar calculation with $\Sigma^{I}$, the second component in the $\zeta$-expansion of $\Upsilon^{I+}$, establishes that it also has unit dilatation weight. This leads to

$$
2 K=k_{\mathrm{D}}^{\mathrm{a}} \partial_{\mathrm{a}} K+k_{\mathrm{D}}^{\overline{\mathrm{a}}} \partial_{\overline{\mathrm{a}}} K
$$

using the definition (3.10) of the Kähler potential.

Next, we establish the $\mathrm{SU}(2)_{R}$ transformation properties of the fields. Consider first the diagonal $\mathrm{U}(1)$ subgroup of $\mathrm{SU}(2)_{R}$ generated by $I^{\underline{1}} \underline{\underline{1}}=-I_{\underline{2}} \cdot{ }^{17}$ It acts as

$$
I^{1}{ }_{1} \phi^{\mathrm{a}}=-\frac{1}{2} k_{\mathrm{D}}^{\mathrm{a}}, \quad I^{1} \underline{1} \bar{\phi}^{\overline{\mathrm{a}}}=+\frac{1}{2} k_{\mathrm{D}}^{\overline{\mathrm{a}}} .
$$

Using $I_{\underline{1}}^{1} \Sigma^{I}=\frac{1}{2} \Sigma^{I}$, it is easy to show that the Kähler potential (3.10) is inert. This implies that $k_{\mathrm{D}}^{\mathrm{a}} \equiv \chi^{\mathrm{a}}$ is a homothetic conformal Killing vector. Now the off-diagonal $\mathrm{SU}(2)_{R}$ component $I_{\underline{2}}=I_{\underline{22}}$ annihilates the antichiral fields and acts on the chiral ones as

$$
I_{\underline{22}} \Phi^{I}=\frac{\partial K}{\partial \Psi_{I}}, \quad I_{22} \Psi_{I}=-\frac{\partial K}{\partial \Phi^{I}} \quad \Longrightarrow \quad I_{22} \phi^{\mathrm{a}}=\omega^{\mathrm{ab}} K_{\mathrm{b}}=\omega_{\overline{\mathrm{b}}}^{\mathrm{a}} \chi^{\overline{\mathrm{b}}} .
$$

Putting these results together, we deduce that

$$
I^{i}{ }_{j} \phi^{\mu}=\left(\mathcal{J}^{i}{ }_{j}\right)^{\mu}{ }_{\nu} \chi^{\nu} .
$$

This implies that the Kähler potential is invariant under all of the $\mathrm{SU}(2)$ generators.

\footnotetext{
${ }^{17}$ This transformation was called the shadow chiral rotation in [44, 55].
} 
At this stage, we should point out how these isometries act on the two-form $\Omega^{++}$. Under dilatations,

$$
\delta_{\mathrm{D}} \Omega^{++}=\Lambda_{\mathrm{D}} \mathcal{L}_{\chi} \Omega^{++}=2 \Lambda_{\mathrm{D}} \Omega^{++} \quad \Longrightarrow \quad \mathcal{L}_{\chi} \omega_{\mathrm{ab}}=2 \omega_{\mathrm{ab}}, \quad \mathcal{L}_{\chi} g_{\mathrm{a} \overline{\mathrm{b}}}=2 g_{\mathrm{a} \overline{\mathrm{b}}},
$$

while $\mathrm{SU}(2)$ transformations rotate the complex structures,

$$
\begin{aligned}
\delta_{\mathrm{SU}(2)} \Omega^{++} & =-\lambda^{++} D^{--} \Omega^{++}+2 \lambda^{0} \Omega^{++}=-2 \lambda^{k}{ }_{i} \Omega_{j k} v^{i+} v^{j+} \Longrightarrow \\
\delta_{\mathrm{SU}(2)} \Omega_{i j} & =-2 \lambda^{k}{ }_{(i} \Omega_{j) k} .
\end{aligned}
$$

This is consistent with the target space transformations

$$
\delta \phi^{\mu}=\Lambda_{\mathrm{D}} \chi^{\mu}+\lambda^{i}{ }_{j}\left(\mathcal{J}^{j}{ }_{i}\right)^{\mu}{ }_{\nu} \chi^{\nu} .
$$

These comprise the isometries required of a hyperkähler cone.

\subsection{Supersymmetry and fermion transformations}

In addition to the $2 n$ complex bosons $\phi^{\mathrm{a}}$ parametrizing the target space, there must be $2 n$ Weyl fermions. It will be convenient for us to define the fermions to be consistent with the $\mathcal{N}=1$ reduction - that is, we will associate one left-handed Weyl fermion with each of the $\phi^{\mathrm{a}}$ and one right-handed Weyl fermion with each of the $\bar{\phi}^{\overline{\mathrm{a}}}$. Using the on-shell superfields $\phi^{\mathrm{a}}$ and $\bar{\phi}^{\overline{\mathrm{a}}}$, we define (using the curved superspace spinor derivatives $\nabla_{\alpha}^{i}$ and $\bar{\nabla}_{\dot{\alpha} i}$ )

$$
\zeta_{\alpha}^{\mathrm{a}}:=\nabla \frac{1}{\alpha} \phi^{\mathrm{a}}, \quad \bar{\zeta}_{\dot{\alpha}}^{\overline{\mathrm{a}}}:=\bar{\nabla}_{\dot{\alpha}} \underline{1} \bar{\phi}^{\overline{\mathrm{a}}} .
$$

The set of fields $\left\{\phi^{\mathrm{a}}, \bar{\phi}^{\overline{\mathrm{a}}}, \zeta_{\alpha}^{\mathrm{a}}, \bar{\zeta}_{\dot{\alpha}}^{\overline{\mathrm{a}}}\right\}$ constitute the on-shell field content of the supersymmetric sigma model. Our goal in this section is to derive their supersymmetry transformations.

We first establish the action of the spinor derivatives on the scalars $\phi^{\mu}: 1^{18}$

$$
\nabla \frac{1}{\alpha} \phi^{\mathrm{a}} \equiv \zeta_{\alpha}^{\mathrm{a}}, \quad \nabla_{\alpha}^{\frac{2}{\alpha}} \phi^{\mathrm{a}}=0, \quad \nabla \frac{1}{\alpha} \bar{\phi}^{\overline{\mathrm{a}}}=0, \quad \nabla \frac{2}{\alpha} \bar{\phi}^{\overline{\mathrm{a}}}=\omega^{\overline{\mathrm{a}}}{ }_{\mathrm{b}} \zeta_{\alpha}^{\mathrm{b}},
$$

and similarly for their complex conjugates. Take the partial pullback of $\Omega^{++}$to the supermanifold, replacing one of the exterior target space derivatives with a spinor derivative:

$$
\begin{aligned}
& \mathrm{d} \phi^{\mu} \partial_{\mu} \Upsilon^{I+} \nabla_{\alpha}^{i} \Gamma_{I}^{+}-\mathrm{d} \phi^{\mu} \partial_{\mu} \Gamma_{I}^{+} \nabla_{\alpha}^{i} \Upsilon^{I+} \\
& =v^{\underline{1}+} v^{\underline{2}+}\left(\frac{1}{\zeta} \mathrm{d} \phi^{\mathrm{a}} \nabla_{\alpha}^{i} \phi^{b} \omega_{\mathrm{ab}}+\mathrm{d} \phi^{\mathrm{a}} \nabla_{\alpha}^{i} \bar{\phi}^{\bar{b}} g_{\mathrm{a} \overline{\mathrm{b}}}+\mathrm{d} \bar{\phi}^{\overline{\mathrm{b}}} \nabla_{\alpha}^{i} \phi^{\mathrm{a}} g_{\mathrm{ab}}+\zeta \mathrm{d} \bar{\phi}^{\overline{\mathrm{a}}} \nabla_{\alpha}^{i} \bar{\phi}^{\overline{\mathrm{b}}} \omega_{\mathrm{ab}}\right) .
\end{aligned}
$$

Now contract with $v_{i}^{+}$and the desired results follow.

The spinor derivatives of $\zeta_{\alpha}^{\mathrm{a}}$ and $\bar{\zeta}_{\dot{\alpha}}^{\bar{a}}$ can be derived directly. Noting, for example, that $\zeta_{\alpha}^{\mathrm{a}}=\nabla \frac{1}{\alpha} \phi^{\mathrm{a}}=-\omega^{\mathrm{a}}{ }_{\mathrm{b}} \nabla_{\alpha}^{\frac{2}{\alpha}} \bar{\phi}^{\overline{\mathrm{b}}}$ one can evaluate any spinor derivative of $\zeta_{\alpha}^{\mathrm{a}}$ by exploiting the fact that each spinor derivative annihilates either $\phi^{\mathrm{a}}$ or $\bar{\phi}^{\overline{\mathrm{b}}}$. This leads to

$$
\begin{array}{rlrl}
\nabla_{\beta}^{1} \zeta_{\alpha}^{\mathrm{a}} & =2 \epsilon_{\beta \alpha} \bar{W}^{r} \omega^{\mathrm{a}}{ }_{\overline{\mathrm{b}}} J_{r}^{\overline{\mathrm{b}}}-\Gamma_{\mathrm{cb}}{ }^{\mathrm{a}} \zeta_{\beta}^{\mathrm{c}} \zeta_{\alpha}^{\mathrm{b}}, & \nabla_{\beta}^{2} \zeta_{\alpha}^{\mathrm{a}} & =2 \epsilon_{\beta \alpha} \bar{W}^{r} J_{r}^{\mathrm{a}}, \\
\bar{\nabla}_{\dot{\beta} \underline{2}} \zeta_{\alpha}^{\mathrm{a}} & =2 i \omega^{\mathrm{a}} \overline{\mathrm{b}}_{\alpha} \nabla_{\alpha \dot{\beta}} \bar{\phi}^{\overline{\mathrm{b}}}-\Gamma_{\mathrm{cd}}{ }^{\mathrm{a}} \omega^{\mathrm{c}} \overline{\overline{\mathrm{c}}} \bar{\zeta}_{\dot{\beta}}^{\overline{\mathrm{c}}} \zeta_{\alpha}^{\mathrm{d}}, & \bar{\nabla}_{\dot{\beta} \underline{1}} \zeta_{\alpha}^{\mathrm{a}}=-2 i \nabla_{\alpha \dot{\beta}} \phi^{\mathrm{a}},
\end{array}
$$

\footnotetext{
${ }^{18}$ Such superfields $\phi^{\mu}$ were called deformed Fayet-Sohnius multiplets in [64].
} 
and similarly for their complex conjugates. The superfield $W^{r}$ is the chiral field strength of the $\mathcal{N}=2$ vector multiplet, involving components $\left\{W^{r}, A_{m}^{r}, \lambda_{\alpha i}^{r}, Y^{r i j}\right\}$. We collect their definitions and supersymmetry transformations in appendix A.

We will also need the dilatation, $\mathrm{U}(1)$, and $\mathrm{SU}(2)_{R}$ transformations of the fermions,

$$
\mathbb{D} \zeta_{\alpha}^{\mathrm{a}}=\frac{3}{2} \zeta_{\alpha}^{\mathrm{a}}-\Gamma_{\mathrm{bc}}{ }^{\mathrm{a}}\left(\mathbb{D} \phi^{\mathrm{b}}\right) \zeta_{\alpha}^{\mathrm{c}}, \quad \mathbb{A} \zeta_{\alpha}^{\mathrm{a}}=-i \zeta_{\alpha}^{\mathrm{a}}, \quad I^{i}{ }_{j} \zeta_{\alpha}^{\mathrm{a}}=-\Gamma_{\mathrm{bc}}{ }^{\mathrm{a}}\left(I^{i}{ }_{j} \phi^{\mathrm{b}}\right) \zeta_{\alpha}^{\mathrm{c}}
$$

and their $S$-supersymmetry transformations,

$$
S_{\beta \underline{1}} \zeta_{\alpha}^{\mathrm{a}}=4 \epsilon_{\beta \alpha} \chi^{\mathrm{a}}, \quad S_{\beta \underline{2}} \zeta_{\alpha}^{\mathrm{a}}=-4 \epsilon_{\beta \alpha} \omega^{\mathrm{a}} \overline{\mathrm{b}} \chi^{\overline{\mathrm{b}}}, \quad \bar{S}^{\dot{\beta} i} \zeta_{\alpha}^{\mathrm{a}}=0 .
$$

All of the matter fields are invariant under the special conformal generator $K_{a}$.

\section{5 $\operatorname{Sp}(n)$-covariant formulation and summary}

We have not commented yet on one important feature of hyperkähler manifolds: the tangent space group is actually $\operatorname{Sp}(n) \times \operatorname{Sp}(1)$ (due to the existence of the covariantly constant holomorphic tensor $\omega_{\mathrm{ab}}$ ) and the $\operatorname{Sp}(1)$ part of the target space connection vanishes. Following $[65,66]$ (see also [3]), we can introduce a tensor $f_{\mu i}{ }^{\mathrm{a}}$ and its inverse $f_{\mathrm{a}}{ }^{i \mu}$, with an $\operatorname{Sp}(n)$ index $\mathrm{a}=1, \cdots, 2 n$. These obey the conditions

$$
f_{\mu i}{ }^{\mathrm{a}} f_{\mathrm{a}}{ }^{i \nu}=\delta_{\mu}{ }^{\nu}, \quad f_{\mathrm{a}}{ }^{i \mu} f_{\mu j}{ }^{\mathrm{b}}=\delta_{\mathrm{a}}{ }^{\mathrm{b}} \delta^{i}{ }_{j}, \quad f_{\mu i}{ }^{\mathrm{a}}=-\epsilon_{i j} \omega^{\mathrm{ab}} g_{\mu \nu} f_{\mathrm{b}}{ }^{j \nu}
$$

and allow one to convert any vector $V^{\mu}$ into an $\operatorname{Sp}(n) \times \operatorname{Sp}(1)$ vector, $V_{i}{ }^{\mathrm{a}}=V^{\mu} f_{\mu i}{ }^{\mathrm{a}}$. They are related to the metric, the hyperkähler two-forms and the complex structures via

$$
g_{\mu \nu}=\epsilon^{i j} \omega_{\mathrm{ab}} f_{\mu i}{ }^{\mathrm{a}} f_{\nu j}{ }^{\mathrm{b}}, \quad\left(\Omega^{i j}\right)_{\mu \nu}=f_{\mu}{ }^{\mathrm{a}(i} f_{\nu}{ }^{\mathrm{b} j)} \omega_{\mathrm{ab}}, \quad\left(\mathcal{J}_{A}\right)^{\mu}{ }_{\nu}=i f_{\nu i}{ }^{\mathrm{a}}\left(\tau_{A}\right)^{i}{ }_{j} f_{\mathrm{a}}{ }^{j \mu}
$$

Requiring $f_{\mu i}$ a to be covariantly constant,

$$
\nabla_{\nu} f_{\mu i}{ }^{\mathrm{a}}:=\partial_{\nu} f_{\mu i}{ }^{\mathrm{a}}-\Gamma_{\nu \mu}{ }^{\rho} f_{\rho i}{ }^{\mathrm{a}}+\Gamma_{\nu \mathrm{b}}{ }^{\mathrm{a}} f_{\mu i}{ }^{\mathrm{b}}=0
$$

defines the $\operatorname{Sp}(n)$ connection $\Gamma_{\mu \mathrm{b}}{ }^{\mathrm{a}}$.

We are actually interested in the situation where the indices $a, b, \cdots$ are not quite flat tangent space $\operatorname{Sp}(n)$ indices, but rather complex world indices in the coordinate system that diagonalizes the complex structure $\mathcal{J}_{3}$. We impose the pseudoreality condition

$$
\left(\mathrm{d} \phi^{\mu} f_{\mu i}{ }^{\mathrm{a}}\right)^{*}=\mathrm{d} \phi^{\mu} f_{\mu}{ }^{i \overline{\mathrm{a}}}=\epsilon^{i j} g^{\overline{\mathrm{a} a}} \omega_{\mathrm{ab}} \mathrm{d} \phi^{\mu} f_{\mu j}{ }^{\mathrm{b}},
$$

where $g_{\mathrm{ab}}$ is the Kähler metric associated with $\mathcal{J}_{3}$. In our conventions, the tensors $f_{\mu i}{ }^{\mathrm{a}}$, $f_{\mu}^{i \overline{\mathrm{a}}}$ and their inverses are given by

$$
\begin{aligned}
& f_{\mu \underline{1}}^{\mathrm{a}}=\delta_{\mu}^{\mathrm{a}}, \quad f_{\mu \underline{\underline{2}}}^{\mathrm{a}}=g_{\mu \mathrm{b}} \omega^{\mathrm{ba}}, \quad f_{\mu}{ }^{\underline{1} \overline{\mathrm{a}}}=\delta_{\mu}{ }^{\overline{\mathrm{a}}}, \quad f_{\mu}{ }^{2} \overline{\mathrm{a}}=g_{\mu \overline{\mathrm{b}}} \omega^{\overline{\mathrm{b}} \overline{\mathrm{a}}}, \\
& f_{\mathrm{a}}{ }^{\mu} \mu=\delta_{\mathrm{a}}{ }^{\mu}, \quad f_{\mathrm{a}}{ }^{\mu} \mu=-\omega_{\mathrm{ab}} g^{\mathrm{b} \mu}, \quad f_{\overline{\bar{a}} \underline{1}}^{\mu}=\delta_{\overline{\mathrm{a}}}{ }^{\mu}, \quad f_{\overline{\bar{a}} \underline{a}^{\mu}}=-\omega_{\overline{\mathrm{a}} \overline{\mathrm{b}}} g^{\overline{\mathrm{b}} \mu},
\end{aligned}
$$

and the $\operatorname{Sp}(n)$ connection is identical to the Kähler connection. 
The advantage of introducing the tensors $f_{\mu i}{ }^{\mathrm{a}}$ is that they simplify the equations given in the preceding sections. For example, the spinor derivatives of $\phi^{\mu}$ in (3.38) become

$$
\nabla_{\alpha}^{i} \phi^{\mu}=\zeta_{\alpha}^{\mathrm{b}} f_{\mathrm{b}}{ }^{i \mu}, \quad \bar{\nabla}_{i}^{\dot{\alpha}} \phi^{\mu}=\bar{\zeta}^{\dot{\alpha} \overline{\mathrm{b}}} f_{\overline{\mathrm{b}} i}{ }^{\mu},
$$

equivalent to the supersymmetry transformations

$$
\delta_{\mathrm{Q}} \phi^{\mu}=\xi_{i} \zeta^{\mathrm{b}} f_{\mathrm{b}}^{i \mu}+\bar{\xi}^{i} \bar{\zeta}^{\overline{\mathrm{b}}}{f_{\overline{\mathrm{b}} i}}^{\mu}
$$

where $\xi_{i}^{\alpha}$ and $\bar{\xi}_{\dot{\alpha}}^{i}$ are the supersymmetry parameters.

Similarly, if we introduce the pseudoreal $\operatorname{Sp}(\mathrm{n}) \times \operatorname{Sp}(1)$ sections $A_{i}{ }^{\mathrm{a}}$ associated with the conformal Killing vectors $\chi^{\mu}[29]$

$$
A_{i}{ }^{\mathrm{a}}:=\chi^{\mu} f_{\mu i}{ }^{\mathrm{a}}, \quad A^{i \overline{\mathrm{a}}}:=\chi^{\mu} f_{\mu}{ }^{i \overline{\mathrm{a}}}, \quad\left(A_{i}^{\mathrm{a}}\right)^{*}=A^{i \overline{\mathrm{a}}}=\epsilon^{i j} \omega^{\overline{\mathrm{a}}}{ }_{\mathrm{b}} A_{j}^{\mathrm{b}},
$$

then the supersymmetry and $S$-supersymmetry transformations of the fermions can be written compactly as

$$
\begin{aligned}
\delta \zeta_{\alpha}^{\mathrm{a}} & =2 i\left(\widehat{\nabla}_{\alpha \dot{\beta}} A_{i}{ }^{\mathrm{a}}\right) \bar{\xi}^{\dot{\beta} i}-2 \bar{W}^{r} J_{r i}{ }^{\mathrm{a}} \epsilon^{i j} \zeta_{\alpha j}-4 \eta_{\alpha}^{i} A_{i}{ }^{\mathrm{a}}-\Gamma_{\mathrm{bc}}{ }^{\mathrm{a}} \delta \phi^{\mathrm{b}} \zeta_{\alpha}^{\mathrm{c}}, \\
\delta \bar{\zeta}^{\dot{\alpha} \overline{\mathrm{a}}} & =2 i\left(\widehat{\nabla}^{\dot{\alpha} \beta} A^{i \overline{\mathrm{a}}}\right) \xi_{\beta i}+2 W^{r} J_{r}{ }^{i \overline{\mathrm{a}}} \epsilon_{i j} \bar{\zeta}^{\dot{\alpha} j}-4 \bar{\eta}_{i}^{\dot{\alpha}} A^{i \overline{\mathrm{a}}}-\Gamma_{\overline{\mathrm{b}} \overline{\mathrm{c}}} \overline{\mathrm{a}} \bar{\phi}^{\overline{\mathrm{b}}} \bar{\zeta}^{\dot{\alpha} \overline{\mathrm{c}}}
\end{aligned}
$$

where $\eta^{i \alpha}$ and $\bar{\eta}_{i \dot{\alpha}}$ are the $S$-supersymmetry parameters, $J_{r i}{ }^{\mathrm{a}}:=J_{r}{ }^{\mu} f_{\mu i}{ }^{\mathrm{a}}$ is the Killing vector associated with the gauged isometries, and $\widehat{\nabla}_{a}$ includes the $\operatorname{Sp}(n)$ connection. For reference, we also give the transformations of the fermions under gauged isometries,

$$
\delta_{g} \zeta_{\alpha}^{\mathrm{a}}=\lambda^{r} \zeta_{\alpha}^{\mathrm{b}} \nabla_{\mathrm{b}} J_{r}^{\mathrm{a}}-\Gamma_{\mathrm{bc}}{ }^{\mathrm{a}} \delta_{g} \phi^{\mathrm{b}} \zeta_{\alpha}^{\mathrm{c}}, \quad \delta_{g} \bar{\zeta}^{\dot{\alpha} \overline{\mathrm{a}}}=\lambda^{r} \bar{\zeta}^{\dot{\alpha} \overline{\mathrm{b}}} \nabla_{\overline{\mathrm{b}}} J_{r}^{\overline{\mathrm{a}}}-\Gamma_{\overline{\mathrm{b}} \overline{\mathrm{c}}}{ }^{\overline{\mathrm{a}}} \delta_{g} \bar{\phi}^{\overline{\mathrm{b}}} \bar{\zeta}^{\dot{\alpha} \overline{\mathrm{c}}}
$$

Note that the scalar fields $\phi^{\mu}$ and the fermions $\zeta_{\alpha}^{\mathrm{b}}$ transform into each other (and into the components of the vector multiplet) under supersymmetry. The conditions (3.38) (equivalently (3.48)) have eliminated the hypermultiplet auxiliary fields and placed the entire multiplet on-shell: in particular, one can check that the supersymmetry algebra closes only up to the equations of motion. These results match those of [29], up to differences in conventions discussed at the end of section 6 .

\section{Building blocks of a component reduction}

In the previous section, we addressed the on-shell structure of the target space multiplets $\phi^{\mu}$, where the auxiliary fields were completely eliminated. In a manifestly supersymmetric setting - which we have implicitly been using - this actually is a stronger condition than what we want. It corresponds, in the $\mathcal{N}=1$ situation, to specifying not only the algebraic equations of motion for the auxiliaries $F^{\mathrm{a}}$ but also the dynamical equations of motion for the physical fields $\phi^{\mathrm{a}}$ and $\zeta_{\alpha}^{\mathrm{a}}$. Our goal in this section now is to describe how to analyze the superfield equations of motion for the arctic multiplet so that only the auxiliary fields are placed on-shell. 


\subsection{Component expansions of the projective multiplets}

We begin by analyzing the component structure of the projective multiplets. The conventional approach is to reduce to $\mathcal{N}=1$ superfields and superspace, but as we have already discussed in the introduction, this is far more difficult in the curved case. Instead, it will be more convenient to reduce directly to component fields. Much insight can be gleaned from the harmonic superspace approach to sigma models (see section 11.4 of [10]), where no $\mathcal{N}=1$ subspace is readily available. The first step is to choose an appropriate system of coordinates. In projective superspace, the following coordinates are well-defined in the north chart and suitable for arctic superfields [46, 47]:

$$
\begin{aligned}
x_{N}^{m} & :=x^{m}-\frac{i}{v^{\underline{1}}+}\left(\theta^{+} \sigma^{m} \bar{\theta}^{\underline{1}}+i \theta^{\underline{1}} \sigma^{m} \bar{\theta}^{+}\right), \quad \theta_{\underline{\alpha}}^{ \pm}:=v_{i}^{ \pm} \theta_{\underline{\alpha}}^{i}, \\
\zeta & :=\frac{v^{\underline{2}}+}{v^{\underline{1}}+}, \quad v^{\underline{1}+}, \quad z_{1}^{--}:=\frac{v^{-}-}{v^{\underline{1}}+}=\frac{v_{\underline{2}}^{-}}{v^{\underline{1}}+}=e^{-2 i \psi} \bar{\zeta} .
\end{aligned}
$$

These lead to the simple expressions

$$
D_{\underline{\alpha}}^{+}=\frac{\partial}{\partial \theta \underline{\alpha-}}, \quad D^{++}=\frac{\partial}{\partial z_{1}^{--}},
$$

implying that holomorphic analytic multiplets are independent of $z_{1}^{--}$and $\theta^{\underline{\alpha}}$. An arctic multiplet $\Upsilon^{I+}$, well-defined in the north chart, is then simply specified as a function $\Upsilon^{I+}\left(x_{N}, v^{\underline{1}}, \zeta, \theta^{+}\right)$possessing an arctic expansion in $\zeta$. Schematically, such a superfield admits a decomposition (following closely the approach of [10])

$$
\begin{aligned}
\Upsilon^{I+}= & \left.\Upsilon^{I+}\right|_{\theta^{+}=0}+\theta^{\alpha+} \Psi_{\alpha}^{I}+\bar{\theta}_{\dot{\alpha}}^{+} \bar{\Psi}^{I \dot{\alpha}}+\left(\theta^{+}\right)^{2} M^{I-}+\left(\bar{\theta}^{+}\right)^{2} N^{I-} \\
& +i \theta^{+} \sigma^{a} \bar{\theta}^{+} A_{a}^{I-}-2\left(\bar{\theta}^{+}\right)^{2} \theta^{\alpha+} \Xi_{\alpha}^{I--}-2\left(\theta^{+}\right)^{2} \bar{\theta}_{\dot{\alpha}}^{+} \bar{\Xi}^{I \dot{\alpha}--}+\left(\theta^{+}\right)^{2}\left(\bar{\theta}^{+}\right)^{2} P^{I(-3)},
\end{aligned}
$$

where the component fields $\Psi_{\underline{\alpha}}^{I}, \cdots, P^{I(-3)}$ depend on $x_{N}, v^{\underline{1}+}$ and $\zeta=v^{\underline{2}+} / v^{\underline{1}+}$. Their dependence on $v^{\underline{1}}$ is indicated by their charge, that is,

$$
\Psi_{\underline{\alpha}}^{I}=\Psi_{\underline{\alpha}}^{I}\left(x_{N}, \zeta\right), \quad \cdots, \quad P^{I(-3)}=\frac{1}{\left(v^{1}+\right)^{3}} P^{I}\left(x_{N}, \zeta\right)
$$

and all expressions are arctic in $\zeta$.

In a curved background, it is more convenient to define the components of $\Upsilon^{I+}$ in a covariant way. The closest analogue to the expansion given above is to define

$$
\begin{aligned}
& \Psi_{\alpha}^{I}:=\frac{1}{v \underline{1}+} \nabla \frac{1}{\alpha} \Upsilon^{I+}, \\
& \Psi_{\dot{\alpha}}^{I}:=\frac{1}{v \underline{\underline{1}}+} \bar{\nabla} \frac{1}{\dot{\alpha}} \Upsilon^{I+}, \\
& M^{I-}:=-\frac{1}{4} \frac{1}{\left(v^{\underline{1}+}\right)^{2}}\left(\nabla^{\underline{1}}\right)^{2} \Upsilon^{I+}, \\
& N^{I-}:=-\frac{1}{4} \frac{1}{\left(v^{\underline{1}}+\right)^{2}}\left(\bar{\nabla}^{\underline{1}}\right)^{2} \Upsilon^{I+} \\
& A_{\alpha \dot{\beta}}^{I-}:=-i \frac{1}{\left(v^{\underline{1}}\right)^{2}} \nabla \frac{1}{\alpha} \nabla_{\dot{\beta}}^{\frac{1}{\dot{ }}} \Upsilon^{I+}, \\
& \Xi_{\alpha}^{I--}:=\frac{1}{8} \frac{1}{\left(v^{\underline{1}}+\right)^{3}} \nabla^{\frac{1}{\alpha}}\left(\bar{\nabla}^{\underline{1}}\right)^{2} \Upsilon^{I+}, \quad \Xi_{\dot{\alpha}}^{I--}:=\frac{1}{8} \frac{1}{\left(v^{\underline{1}+}\right)^{3}} \bar{\nabla}_{\dot{\alpha}}^{\frac{1}{\dot{\alpha}}}\left(\nabla^{\underline{1}}\right)^{2} \Upsilon^{I+}, \\
& P^{I(-3)}:=\frac{1}{16} \frac{1}{\left(v^{\underline{1}+}\right)^{4}}\left(\nabla^{\underline{1}}\right)^{2}\left(\bar{\nabla}^{\underline{1}}\right)^{2} \Upsilon^{I+} \text {. }
\end{aligned}
$$


Each of these components is manifestly holomorphic and well-defined in the north chart. The corresponding formulae for the components of an antarctic multiplet $\breve{\Upsilon}^{\bar{I}+}$ are found by replacing $v^{\underline{1}+} \rightarrow v^{\underline{2}+}$ and $\nabla_{\underline{\alpha}}^{\frac{1}{\alpha}} \rightarrow \nabla_{\underline{\alpha}}^{2}$.

An inconvenient feature of these component fields is that the arctic and antarctic fields are naturally defined in terms of different sets of spinor derivatives. Moreover, when we actually analyze the component action, we will encounter the spinor derivatives $\nabla_{\underline{\alpha}}^{-}:=$ $v_{i}^{-} \nabla_{\underline{\alpha}}^{i}$, which involve a linear combination of $\nabla_{\underline{\alpha}}^{1}$ and $\nabla_{\underline{\alpha}}^{2}$. Using $\nabla_{\underline{\alpha}}^{-}=\frac{1}{v \underline{1}+} \nabla_{\underline{\alpha}}^{1}+z_{1}^{--} \nabla_{\underline{\alpha}}^{+}$, it is straightforward to determine the relation between expressions like $\nabla_{\underline{\alpha}}^{-} \Upsilon^{I+}$ and $\nabla_{\underline{\alpha}}^{\underline{1}} \Upsilon^{I+}$. The expressions for the lowest few components are rather simple:

$$
\begin{aligned}
\nabla_{\underline{\alpha}}^{-} \Upsilon^{I+} & =\Psi_{\underline{\alpha}}^{I}, & \nabla_{\alpha}^{-} \bar{\nabla}_{\dot{\beta}}^{-} \Upsilon^{I+} & =i A_{\alpha \dot{\beta}}^{I-}-2 i z_{1}^{--} \nabla_{\alpha \dot{\beta}} \Upsilon^{I+}, \\
-\frac{1}{4}\left(\bar{\nabla}^{-}\right)^{2} \Upsilon^{I+} & =N^{I-}-z_{1}^{--} W^{r} \mathcal{J}_{r}^{I+}, & -\frac{1}{4}\left(\nabla^{-}\right)^{2} \Upsilon^{I+} & =M^{I-}-z_{1}^{--} \bar{W}^{r} \mathcal{J}_{r}^{I+} .
\end{aligned}
$$

Recall that the arctic function $\mathcal{J}_{r}^{I+}$ arises from the action of the gauge generator on $\Upsilon^{I+}$, so some of the terms in the second line above are present only when isometries are gauged. The expressions with three spinor derivatives are

$$
\begin{aligned}
\frac{1}{8} \nabla_{\alpha}^{-}\left(\bar{\nabla}^{-}\right)^{2} \Upsilon^{I+}= & \Xi_{\alpha}^{I--}+z_{1}^{--}\left(-\frac{i}{2} \nabla_{\alpha \dot{\beta}} \bar{\nabla}^{\dot{\beta}-} \Upsilon^{I+}+2 \lambda_{\alpha}^{r-} \mathcal{J}_{r}^{I+}+\frac{1}{2} W^{r} \nabla_{\alpha}^{-} \mathcal{J}_{r}^{I+}\right. \\
& \left.-\frac{1}{2} W_{\alpha}^{\beta} \nabla_{\beta}^{-} \Upsilon^{I+}-\frac{3}{2} \chi_{\alpha}^{-} \Upsilon^{I+}+\frac{3}{2} \chi_{\alpha}^{+} D^{--} \Upsilon^{I+}\right) \\
& -\left(z_{1}^{--}\right)^{2} \lambda_{\alpha}^{r+} \mathcal{J}_{r}^{I+} \\
\frac{1}{8} \bar{\nabla}^{\dot{\alpha}-}\left(\nabla^{-}\right)^{2} \Upsilon^{I+}= & \bar{\Xi}_{\dot{\alpha}}^{I--}+z_{1}^{--}\left(\frac{i}{2} \nabla^{\dot{\alpha} \beta} \nabla_{\beta}^{-} \Upsilon^{I+}-2 \bar{\lambda}^{\dot{\alpha} r-} \mathcal{J}_{r}^{I+}+\frac{1}{2} \bar{W}^{r} \bar{\nabla}^{\dot{\alpha}-} \mathcal{J}_{r}^{I+}\right. \\
& \left.-\frac{1}{2} \bar{W}_{\dot{\beta}}^{\dot{\alpha}} \bar{\nabla}^{\dot{\beta}-} \Upsilon^{I+}+\frac{3}{2} \bar{\chi}^{\dot{\alpha}-} \Upsilon^{I+}-\frac{3}{2} \bar{\chi}^{\dot{\alpha}+} D^{--} \Upsilon^{I+}\right) \\
& +\left(z_{1}^{--}\right)^{2} \bar{\lambda}^{\dot{\alpha} r+} \mathcal{J}_{r}^{I+}
\end{aligned}
$$

and involve the covariant conformal supergravity fields $W_{\alpha \beta}$ and $\chi_{\alpha i}$ as well as the gaugino $\lambda_{\alpha i}^{r}$. The term with four spinor derivatives is the most complicated:

$$
\begin{aligned}
\frac{1}{16}\left(\nabla^{-}\right)^{2}\left(\bar{\nabla}^{-}\right)^{2} \Upsilon^{I+}= & P^{I(-3)}+z_{1}^{--}\left\{-\frac{i}{2} \nabla^{\dot{\alpha} \alpha} \nabla_{\alpha}^{-} \nabla_{\dot{\alpha}}^{-} \Upsilon^{I+}-3 D D^{--} \Upsilon^{I+}\right. \\
& +\frac{3}{2} \chi^{\alpha+} D^{--} \nabla_{\alpha}^{-} \Upsilon^{I+}-\frac{3}{2} \bar{\chi}_{\dot{\alpha}}^{+} D^{--} \bar{\nabla}^{\dot{\alpha}-} \Upsilon^{I+} \\
& +2 \lambda^{\alpha r-} \nabla_{\alpha}^{-} \mathcal{J}_{r}^{I+}-2 \bar{\lambda}_{\dot{\alpha}}^{r-} \bar{\nabla}^{\dot{\alpha}-} \mathcal{J}_{r}^{I+} \\
& \left.+\frac{1}{4} \bar{W}^{r}\left(\bar{\nabla}^{-}\right)^{2} \mathcal{J}_{r}^{I+}+\frac{1}{4} W^{r}\left(\nabla^{-}\right)^{2} \mathcal{J}_{r}^{I+}+3 Y^{r--} \mathcal{J}_{r}^{I+}\right\} \\
& +\left(z_{1}^{--}\right)^{2}\left\{\frac{3}{2} D \Upsilon^{I+}-\lambda^{\alpha+} \nabla_{\alpha}^{-} \mathcal{J}_{r}^{I+}+\bar{\lambda}_{\dot{\alpha}}^{r+} \bar{\nabla}^{\dot{\alpha}-} \mathcal{J}_{r}^{I+}\right. \\
& \left.-\frac{1}{2} W^{r} \bar{W}^{s}\left\{X_{r}, X_{s}\right\} \Upsilon^{I+}-3 Y^{r-+} \mathcal{J}_{r}^{I+}+\frac{1}{2} \nabla_{\alpha \dot{\alpha}} \nabla^{\dot{\alpha} \alpha} \Upsilon^{I+}\right\} \\
& +\left(z_{1}^{--}\right)^{3} Y^{r++} \mathcal{J}_{r}^{I+} .
\end{aligned}
$$




\begin{tabular}{|clc|}
\hline component e.o.m. & $\Longrightarrow$ & constrained field \\
\hline$F^{\mathrm{a}}$ & $\Longrightarrow$ & $\bar{D}^{2} K_{\mathrm{a}} \sim g_{\mathrm{a} \overline{\mathrm{b}}} \bar{F}^{\overline{\mathrm{b}}}$ \\
$\zeta_{\alpha}^{\mathrm{a}}$ & $\Longrightarrow$ & $D_{\alpha} \bar{D}^{2} K_{\mathrm{a}} \sim g_{\mathrm{a} \overline{\mathrm{b}}} \partial_{\alpha \dot{\alpha}} \bar{\zeta} \bar{\zeta}^{\mathrm{b}}$ \\
$\phi^{\mathrm{a}}$ & $\Longrightarrow$ & $D^{2} \bar{D}^{2} K_{\mathrm{a}} \sim g_{\mathrm{a} \overline{\mathrm{b}}} \square \phi^{\overline{\mathrm{b}}}$ \\
\hline
\end{tabular}

Table 1. $\mathcal{N}=1$ component equations of motion.

Similar equations to (4.6) arise if we replace the arctic $\Upsilon^{I+}$ with the antarctic $\breve{\Upsilon}^{\bar{I}+}$ 一 the only change is the replacement of $z_{1}^{--}$with $z_{2}^{--}:=v^{-2}-v^{2+}$.

\subsection{Auxiliary field equations of motion}

Now we need to understand what conditions arise from placing only the auxiliary fields on-shell. It helps to recall again the rigid $\mathcal{N}=1$ situation where, in the absence of a superpotential, the on-shell equation of motion is given by

$$
\int \mathrm{d}^{4} x \mathrm{~d}^{2} \theta\left(\bar{D}^{2} K_{\mathrm{a}}\right) \delta \phi^{\mathrm{a}}=0 \quad \Longrightarrow \quad \bar{D}^{2} K_{\mathrm{a}}=0
$$

The chiral superfield $\bar{D}^{2} K_{\mathrm{a}}$ is constrained to vanish. At the component level, this corresponds to three distinct equations, corresponding to the equations of motion of the three components of $\phi^{\mathrm{a}}$. Setting to zero the lowest component of $\bar{D}^{2} K_{\mathrm{a}}$ amounts to constraining the auxiliary field, while constraining the higher two components leads to dynamical equations of motion. The situation can be rendered schematically as in table 1 .

These statements apply equally well in the $\mathcal{N}=2$ setting. There we have the equations (3.1), equivalently written as

$$
-\frac{1}{2 \pi} \oint_{\mathcal{C}} \mathrm{d} \tau \int \mathrm{d}^{4} x \mathrm{~d}^{4} \theta^{+} \mathcal{E}^{--} \Gamma_{I}^{+} \delta \Upsilon^{I+}=0 \quad \Longrightarrow \quad \Gamma_{I}^{+} \text {arctic }
$$

which place the arctic multiplet on-shell and imply that the composite $\Gamma_{I}^{+}$is an arctic multiplet. If we introduce the component fields $\Psi_{\underline{\alpha}}, \cdots, P_{I}^{(-3)}$ for $\Gamma_{I}^{+}$in analogy to (4.5), then the equations of motion for each of the $\Upsilon^{I+}$ component fields implies the corresponding arctic nature of these components of $\Gamma_{I}^{+}$. For example, by considering the component reduction of (4.8), the equation of motion for the highest component $P^{I(-3)}$ of $\Upsilon^{I+}$ must set the lowest component of $\Gamma_{I}^{+}$to be arctic, and similarly throughout the multiplet. The precise relations are given in table 2 .

The key issue here is that the auxiliary field equations consist of all but the final two lines of table 2, which for dimensional reasons must contain the field equations for the physical fermions and scalars. Keeping in mind that $\Gamma_{I}^{+}$is a composite quantity, it follows that setting the components $\Gamma_{I}^{+}$through $N_{I}^{-}$to be arctic must correspond to fixing them and the original quantities $\Upsilon^{I+}$ through $N^{I-}$ to be given by their on-shell expressions. For the on-shell superfields discussed in the previous section, these expressions are easy to work out. We already know that the lowest components $\phi^{\mathrm{a}}=\left(\Phi^{I}, \Psi_{I}\right)$ are defined by (3.3). The 


\begin{tabular}{|clc|}
\hline component e.o.m. & $\Longrightarrow$ & constrained arctic field \\
\hline$P^{I(-3)}$ & $\Longrightarrow$ & $\Gamma_{I}^{+}$ \\
$\Xi_{\underline{\alpha}}^{I--}$ & $\Longrightarrow$ & $\Psi_{I \underline{\alpha}}$ \\
$N^{I-}$ & $\Longrightarrow$ & $M_{I}^{-}$ \\
$A_{\alpha \dot{\alpha}}^{I-}$ & $\Longrightarrow$ & $A_{I \alpha \dot{\alpha}}^{-}$ \\
$M^{I-}$ & $\Longrightarrow$ & $N_{I}^{-}$ \\
$\Psi_{\underline{\alpha}}^{I}$ & $\Longrightarrow$ & $\Xi_{I-\alpha}^{--}$ \\
$\Upsilon^{I+}$ & $\Longrightarrow$ & $P_{I}^{(-3)}$ \\
\hline
\end{tabular}

Table 2. $\mathcal{N}=2$ component equations of motion.

components at next order in $\theta$ are

$$
\begin{aligned}
& \Psi_{\alpha}^{I}=\frac{1}{v^{\underline{1}+}} \zeta_{\alpha}^{\mathrm{b}} \partial_{\mathrm{b}} \Upsilon^{I+}=-\frac{1}{v^{2}+} \zeta_{\alpha}^{\mathrm{b}} \omega_{\mathrm{b}}^{\overline{\mathrm{b}}} \partial_{\overline{\mathrm{b}}} \Upsilon^{I+}, \\
& \Psi_{\dot{\alpha}}^{I}=-\frac{1}{v^{2}+} \bar{\zeta}_{\dot{\alpha}}^{\overline{\mathrm{b}}} \partial_{\overline{\mathrm{b}}} \Upsilon^{I+}=-\frac{1}{v^{1+}+} \bar{\zeta}_{\dot{\alpha}}^{\overline{\mathrm{b}}} \omega_{\overline{\mathrm{b}}}^{\mathrm{b}} \partial_{\mathrm{b}} \Upsilon^{I+} .
\end{aligned}
$$

Analogous expressions follow for $\Psi_{I \underline{\alpha}}$ by replacing $\Upsilon^{I+}$ with $\Gamma_{I}^{+}$. The $\theta^{2}$ components are

$$
\begin{aligned}
& A_{\alpha \dot{\alpha}}^{I-}=\frac{2}{v^{\underline{1}+} v^{2}+} \nabla_{\alpha \dot{\alpha}} \bar{\phi}^{\overline{\mathrm{b}}} \partial_{\overline{\mathrm{b}}} \Upsilon^{I+}+\frac{i}{v^{+}+v^{2}+} \zeta_{\alpha}^{\mathrm{b}} \bar{\zeta}_{\dot{\mathrm{\alpha}}}^{\overline{\mathrm{b}}} \partial_{\mathrm{b}} \partial_{\overline{\mathrm{b}}} \Upsilon^{I+}, \\
& A_{\alpha \dot{\alpha}}^{\bar{I}-}=-\frac{2}{v^{\underline{1}+} v^{\underline{2}}+} \nabla_{\alpha \dot{\alpha}} \phi^{\mathrm{b}} \partial_{\mathrm{b}} \breve{\Upsilon}^{I+}+\frac{i}{v^{\underline{1}+} v^{\underline{2}+}} \zeta_{\alpha}^{\mathrm{b}} \bar{\zeta}_{\dot{\alpha}}^{\overline{\mathrm{b}}} \partial_{\mathrm{b}} \partial_{\overline{\mathrm{b}}} \breve{\Upsilon}^{I+},
\end{aligned}
$$

and

$$
\begin{aligned}
M^{I-} & =\frac{1}{v^{\underline{1}+} v^{\underline{2}}}\left(\bar{W}^{r} \bar{J}_{r}^{\overline{\mathrm{b}}} \partial_{\overline{\mathrm{b}}} \Upsilon^{I+}+\frac{1}{4} \zeta^{\alpha \mathrm{a}} \zeta_{\alpha}^{\mathrm{b}} \omega_{\mathrm{b}}^{\overline{\mathrm{b}}} \partial_{\mathrm{a}} \partial_{\overline{\mathrm{b}}} \Upsilon^{I+}\right), \\
N^{I-} & =\frac{1}{v^{\underline{1}+} v^{\underline{2}+}}\left(W^{r} \bar{J}_{r}^{\overline{\mathrm{b}}} \partial_{\overline{\mathrm{b}}} \Upsilon^{I+}-\frac{1}{4} \bar{\zeta}_{\dot{\alpha}}^{\overline{\mathrm{a}}} \bar{\zeta}^{\dot{\alpha} \overline{\mathrm{b}}} \omega_{\overline{\mathrm{b}}}^{\mathrm{b}} \partial_{\overline{\mathrm{a}}} \partial_{\mathrm{b}} \Upsilon^{I+}\right), \\
M^{\bar{I}-} & =\frac{1}{v^{\underline{1}+} v^{\underline{2}+}}\left(-\bar{W}^{r} J_{r}^{\mathrm{b}} \partial_{\mathrm{b}} \breve{\Upsilon}^{\bar{I}+}+\frac{1}{4} \zeta^{\alpha \mathrm{a}} \zeta_{\alpha}^{\mathrm{b}} \omega_{\mathrm{b}}^{\overline{\mathrm{b}}} \partial_{\mathrm{a}} \partial_{\overline{\mathrm{b}}} \breve{\Upsilon}^{\bar{I}+}\right), \\
N^{\bar{I}-} & =\frac{1}{v^{\underline{1}+} v^{\underline{2}}}\left(-W^{r} J_{r}^{\mathrm{b}} \partial_{\mathrm{b}} \breve{\Upsilon}^{I+}-\frac{1}{4} \bar{\zeta}_{\dot{\alpha}}^{\overline{\mathrm{a}}} \bar{\zeta}^{\dot{\alpha} \overline{\mathrm{b}}} \omega_{\overline{\mathrm{b}}}^{\mathrm{b}} \partial_{\overline{\mathrm{a}}} \partial_{\mathrm{b}} \breve{\Upsilon}^{I+}\right) .
\end{aligned}
$$

These conditions, as well as the corresponding ones for the components of $\Gamma_{I}^{+}$and $\bar{\Gamma}_{\bar{I}}^{+}$, constitute the set of auxiliary equations of motion. We cannot specify the components $\Xi_{\underline{\alpha}}^{I--}$ and $P^{I(-3)}$ without placing the physical fields on-shell.

\subsection{Some relevant quantities}

In performing the component reduction, there are a number of geometric quantities that we will encounter that arise from simple expressions in terms of the arctic superfields. Rather than discuss them piecemeal, one at a time as we come across them, we present them here together to emphasize their common features. They can be grouped loosely into 
three classes: quantities corresponding to pullbacks of the hyperkähler two-forms, which exist even in the flat limit; quantities associated with gauged isometries; and quantities that arise from the cone structure.

\subsubsection{Hyperkähler two-forms and their pullbacks}

Recall that the hyperkähler two-forms can be represented equivalently as

$$
\begin{aligned}
\Omega^{++} & =\mathrm{d} \Upsilon^{I+} \wedge \mathrm{d} \Gamma_{I}^{+}=\mathrm{d} \breve{\Upsilon}^{\bar{J}+} \wedge \mathrm{d} \breve{\Gamma}_{\bar{J}}^{+} \\
& =v^{\underline{1}+} v^{\underline{2}+}\left(\frac{1}{2 \zeta} \mathrm{d} \phi^{\mathrm{a}} \wedge \mathrm{d} \phi^{\mathrm{b}} \omega_{\mathrm{ab}}+\mathrm{d} \phi^{\mathrm{a}} \wedge \mathrm{d} \bar{\phi}^{\overline{\mathrm{b}}} g_{\mathrm{a} \overline{\mathrm{b}}}+\frac{\zeta}{2} \mathrm{~d} \bar{\phi}^{\overline{\mathrm{a}}} \wedge \mathrm{d} \bar{\phi}^{\overline{\mathrm{b}}} \omega_{\overline{\mathrm{a}} \overline{\mathrm{b}}}\right)
\end{aligned}
$$

We can interpret the second line as a superfield expression with on-shell superfields $\phi^{\mathrm{a}}$ provided we only work at most to order $\theta^{2}$. Taking the pullback to the supermanifold and using two spinor derivatives of opposite chirality, we find

$$
\begin{aligned}
\Omega_{\alpha \dot{\beta}} & :=\nabla_{\alpha}^{-} \Upsilon^{I+} \bar{\nabla}_{\dot{\beta}}^{-} \Gamma_{I}^{+}-\nabla_{\alpha}^{-} \Gamma_{I}^{+} \bar{\nabla}_{\dot{\beta}}^{-} \Upsilon^{I+}=\nabla_{\alpha}^{-} \breve{\Upsilon}^{\bar{I}+} \bar{\nabla}_{\dot{\beta}}^{-} \breve{\Gamma}_{\bar{I}}^{+}-\nabla_{\alpha}^{-} \breve{\Gamma}_{\bar{I}}^{+} \bar{\nabla}_{\dot{\beta}}^{-} \breve{\Upsilon}^{\bar{I}+}, \\
& =-\frac{1}{v \underline{\underline{1}+} v^{2}+} \zeta_{\alpha}^{\mathrm{a}} \bar{\zeta}_{\dot{\beta}}^{\overline{\mathrm{b}}}\left(\partial_{\mathrm{a}} \Upsilon^{I+} \partial_{\overline{\mathrm{b}}} \Gamma_{I}^{+}-\partial_{\mathrm{a}} \Gamma_{I}^{+} \partial_{\overline{\mathrm{b}}} \Upsilon^{I+}\right)=-\zeta_{\alpha}^{\mathrm{a}} \bar{\zeta}_{\dot{\beta}}^{\overline{\mathrm{b}}} g_{\mathrm{a} \overline{\mathrm{b}}},
\end{aligned}
$$

where the second line follows from (4.9) and (3.9b). Similar expressions for other quantities can be derived:

$$
\begin{aligned}
& \Omega_{\alpha \beta}:=\nabla_{\alpha}^{-} \Upsilon^{I+} \nabla_{\beta}^{-} \Gamma_{I}^{+}-\nabla_{\alpha}^{-} \Gamma_{I}^{+} \nabla_{\beta}^{-} \Upsilon^{I+}=\zeta_{\alpha}^{\mathrm{a}} \zeta_{\beta}^{\mathrm{b}} \omega_{\mathrm{ab}}, \\
& \Omega_{\dot{\alpha} \dot{\beta}}:=\nabla_{\dot{\alpha}}^{-} \Upsilon^{I+} \nabla_{\dot{\beta}}^{-} \Gamma_{I}^{+}-\nabla_{\dot{\alpha}}^{-} \Gamma_{I}^{+} \nabla_{\dot{\beta}}^{-} \Upsilon^{I+}=\zeta_{\dot{\alpha}}^{\overline{\mathrm{a}}} \zeta_{\dot{\beta}}^{\overline{\mathrm{b}}} \omega_{\overline{\mathrm{a}} \overline{\mathrm{b}}} .
\end{aligned}
$$

Note that each of the quantities $\Omega_{\alpha \beta}, \Omega_{\alpha \dot{\beta}}$ and $\Omega_{\dot{\alpha} \dot{\beta}}$ are harmonic-independent at lowest order in their $\theta$ expansion. Similar pullbacks can be defined using the vector derivatives,

$$
\begin{aligned}
& \Omega_{\alpha b}^{+}:=\nabla_{\alpha}^{-} \Upsilon^{I+} \nabla_{b} \Gamma_{I}^{+}-\nabla_{\alpha}^{-} \Gamma_{I}^{+} \nabla_{b} \Upsilon^{I+}=v^{\underline{1}+} \zeta_{\alpha}^{\mathrm{a}} \omega_{\mathrm{ab}} \nabla_{b} \phi^{\mathrm{b}}+v^{\underline{2}+} \zeta_{\alpha}^{\mathrm{a}} g_{\mathrm{a} \overline{\mathrm{b}}} \nabla_{b} \phi^{\overline{\mathrm{b}}} \\
& \Omega_{\dot{\alpha} b}^{+}:=\nabla_{\dot{\alpha}}^{-} \Upsilon^{I+} \nabla_{b} \Gamma_{I}^{+}-\nabla_{\dot{\alpha}}^{-} \Gamma_{I}^{+} \nabla_{b} \Upsilon^{I+}=-v^{\underline{2}+} \bar{\zeta}_{\dot{\alpha}}^{\overline{\mathrm{a}}} \omega_{\overline{\mathrm{a}} \overline{\mathrm{b}}} \nabla_{b} \phi^{\overline{\mathrm{b}}}+v^{\underline{1}+} \bar{\zeta}_{\dot{\alpha}}^{\overline{\mathrm{a}}} g_{\overline{\mathrm{a} b}} \nabla_{b} \phi^{\mathrm{b}},
\end{aligned}
$$

or with the gauge generator,

$$
\begin{aligned}
& \Omega_{r \beta}^{+}:=X_{r} \Upsilon^{I+} \nabla_{\beta}^{-} \Gamma_{I}^{+}-X_{r} \Gamma_{I}^{+} \nabla_{\beta}^{-} \Upsilon^{I+}=v^{1+} J_{r}^{\mathrm{a}} \omega_{\mathrm{ab}} \zeta_{\beta}^{\mathrm{b}}-v^{2+} J_{r}^{\overline{\mathrm{a}}} g_{\overline{\mathrm{a}} \mathrm{b}} \zeta_{\beta}^{\mathrm{b}}, \\
& \Omega_{r \dot{\beta}}^{+}:=X_{r} \Upsilon^{I+} \bar{\nabla}_{\dot{\beta}}^{-} \Gamma_{I}^{+}-X_{r} \Gamma_{I}^{+} \bar{\nabla}_{\dot{\beta}}^{-} \Upsilon^{I+}=-v^{\underline{2}} J_{r}^{\overline{\mathrm{a}}} \omega_{\overline{\mathrm{a}} \overline{\mathrm{b}}} \bar{\zeta}_{\dot{\beta}}^{\overline{\mathrm{b}}}-v^{+}{ }_{r}^{\mathrm{a}} g_{\mathrm{a} \overline{\mathrm{b}}} \bar{\zeta}_{\dot{\beta}}^{\overline{\mathrm{b}}}
\end{aligned}
$$

It is also possible to define other pullbacks such as $\Omega_{r b}^{++}$or $\Omega_{a b}^{++}$, but these will not play a major role in our discussion so we do not give their explicit forms.

It can be useful to employ a condensed notation to simplify the right-hand sides of (4.15) and (4.16). Using the $\operatorname{Sp}(\mathrm{n})$ vielbeins $f_{\mu i}{ }^{\mathrm{a}}$ introduced in section 3.5, we define

$$
\zeta_{\beta}^{i \mu}:=\nabla_{\beta}^{i} \phi^{\mu}=\zeta_{\beta}^{\mathrm{a}} f_{\mathrm{a}}{ }^{i \mu}, \quad \bar{\zeta}_{\dot{\beta} i}{ }^{\mu}:=\bar{\nabla}_{\dot{\beta} i} \phi^{\mu}=\bar{\zeta}_{\dot{\beta}}^{\overline{\mathrm{a}}} f_{\overline{\mathrm{a}} i}{ }^{\mu}
$$

Then one can alternatively write (using also the fields $A_{i}{ }^{\mathrm{a}}$ and $J_{r i}{ }^{\mathrm{a}}$ ),

$$
\begin{array}{ll}
\Omega_{\alpha b}^{+}=-\zeta_{\alpha}^{+\mu} g_{\mu \nu} \nabla_{b} \phi^{\nu}=-\zeta_{\alpha}^{\mathrm{a}} \nabla_{b} A^{+\overline{\mathrm{b}}} g_{\mathrm{a} \overline{\mathrm{b}}}, & \Omega_{\dot{\alpha} b}^{+}=-\bar{\zeta}_{\dot{\alpha}}^{+\mu} g_{\mu \nu} \nabla_{b} \phi^{\nu}=-\bar{\zeta}_{\dot{\alpha}}^{\overline{\mathrm{a}}} \nabla_{b} A^{+\mathrm{b}} g_{\overline{\mathrm{a}} \mathrm{b}} \\
\Omega_{r \beta}^{+}=\zeta_{\alpha}^{+\mu} J_{r \mu}=\zeta_{\alpha}^{\mathrm{a}} J_{r}^{+\overline{\mathrm{b}}} g_{\mathrm{a} \overline{\mathrm{b}}}, & \Omega_{r \dot{\beta}}^{+}=\bar{\zeta}_{\dot{\alpha}}^{+\mu} J_{r \mu}=\bar{\zeta}_{\dot{\alpha}}^{\overline{\mathrm{a}}} J_{r}^{+\mathrm{b}} g_{\overline{\mathrm{a}} \mathrm{b}} .
\end{array}
$$


It is also useful to note some relations between these various quantities. For example, $\nabla_{\beta}^{j} \Omega_{\alpha \dot{\alpha}}$ and $\bar{\nabla}_{\dot{\beta}}^{j} \Omega_{\alpha \dot{\alpha}}$ are found by taking

$$
\begin{array}{lll}
\nabla_{\beta}^{+} \Omega_{\alpha \dot{\alpha}}=2 \epsilon_{\beta \alpha} \bar{W}^{s} \Omega_{s \dot{\alpha}}^{+}+2 i \Omega_{\alpha \beta \dot{\alpha}}^{+} & \Longrightarrow & \nabla_{\beta}^{j} \Omega_{\alpha \dot{\alpha}}=2 \epsilon_{\beta \alpha} \bar{W}^{s} \Omega_{s \dot{\alpha}}^{j}+2 i \Omega_{\alpha \beta \dot{\alpha}}^{j}, \\
\bar{\nabla}_{\dot{\beta}}^{+} \Omega_{\alpha \dot{\alpha}}=-2 \epsilon_{\dot{\beta} \dot{\alpha}} W^{r} \Omega_{r \alpha}^{+}-2 i \Omega_{\dot{\alpha} \alpha \dot{\beta}}^{+} & \Longrightarrow & \bar{\nabla}_{\dot{\beta}}^{j} \Omega_{\alpha \dot{\alpha}}=-2 \epsilon_{\dot{\beta} \dot{\alpha}} W^{r} \Omega_{r \alpha}^{j}-2 i \Omega_{\dot{\alpha} \alpha \dot{\beta}}^{j} .
\end{array}
$$

Relations such as these will be extremely useful in our analysis.

\subsubsection{Components of the $\mathcal{N}=2$ moment map}

Recall that the $\mathcal{N}=2$ moment map $D_{r}^{++}$is given in projective superspace by (3.18). With the auxiliary field equations imposed, $D_{r}^{++}$is a globally defined superfield to order $\theta^{2}$. Its lowest component was given already in (3.22). Its higher components can be analyzed by applying spinor derivatives to (3.18). A single spinor derivative gives

$$
\nabla_{\underline{\alpha}}^{+} D_{r}^{++}=0, \quad \nabla_{\underline{\alpha}}^{-} D_{r}^{++}=\Omega_{r \underline{\alpha}}^{+} .
$$

For two spinor derivatives, one finds

$$
\left(\nabla^{-}\right)^{2} D_{r}^{++}=-4 \bar{W}^{s} f_{s r}{ }^{t} D_{t}^{+-}-2 \bar{W}^{s} J_{s}^{\mu} J_{r \mu}-\zeta^{\mathrm{a}} \zeta^{\mathrm{b}} \nabla_{\mathrm{a}}\left(\omega_{\mathrm{bc}} J_{r}^{\mathrm{c}}\right),
$$

which implies

$$
\frac{1}{3} \nabla_{i j} D_{r}^{i j}=-2 \bar{W}^{s} J_{s}^{\mu} J_{r \mu}-\zeta^{\mathrm{a}} \zeta^{\mathrm{b}} \nabla_{\mathrm{a}}\left(\omega_{\mathrm{bc}} J_{r}^{\mathrm{c}}\right) .
$$

\subsubsection{Hyperkähler cone potential and hyperkähler one-forms}

Recall that the hyperkähler cone possesses a globally defined function, the hyperkähler potential $K$. In terms of superfield quantities, $K$ is given by the pullback of $\Omega^{++}$onto the auxiliary $\mathrm{SU}(2)$ manifold,

$$
K:=D^{--} \Upsilon^{I+} D^{0} \Gamma_{I}^{+}-D^{0} \Upsilon^{I+} D^{--} \Gamma_{I}^{+}=\Gamma_{I}^{+} D^{--} \Upsilon^{I+}-\Upsilon^{I+} D^{--} \Gamma_{I}^{+}
$$

Let us prove this result. Because $\mathcal{F}^{++}$is independent of $v^{i+}$ in the superconformal case, this expression is equivalent to its conjugate so $K$ is real. It is easy to check that $D^{++} K=0$. Because $K$ is both arctic and antarctic, it must actually be harmonic-independent. To prove that it is the hyperkähler potential, observe that an $\mathrm{SU}(2)_{R}$ transformation acts on projective multiplets and on the target space respectively as

$$
\lambda^{i}{ }_{j} I^{j}{ }_{i}=-\lambda^{++} D^{--}+\lambda^{0} D^{0}+\lambda^{--} D^{++}=\lambda^{i}{ }_{j}\left(\mathcal{J}^{j}{ }_{i}\right)^{\mu}{ }_{\nu} \chi^{\nu} \partial_{\mu} .
$$

For projective multiplets $\Upsilon^{I+}$ and $\Gamma_{I}^{+}$, this implies

$$
D^{--}=\left(\mathcal{J}^{--}\right)^{\mu}{ }_{\nu} \chi^{\nu} \partial_{\mu}, \quad D^{0}=2\left(\mathcal{J}^{-+}\right)^{\mu}{ }_{\nu} \chi^{\nu} \partial_{\mu}, \quad D^{++}=-\left(\mathcal{J}^{++}\right)^{\mu}{ }_{\nu} \chi^{\nu} \partial_{\mu} .
$$

It follows that (4.23) can be written

$$
\begin{aligned}
K & =2\left(\mathcal{J}^{--}\right)^{\mu}{ }_{\nu} \chi^{\nu}\left(\mathcal{J}^{-+}\right)^{\rho}{ }_{\sigma} \chi^{\sigma}\left(\partial_{\mu} \Upsilon^{I+} \partial_{\rho} \Gamma_{I}^{+}-\partial_{\mu} \Gamma_{I}^{+} \partial_{\rho} \Upsilon^{I+}\right) \\
& =-2 \chi_{\nu}\left(\mathcal{J}^{--} \mathcal{J}^{++} \mathcal{J}^{-+}\right)^{\nu}{ }_{\sigma} \chi^{\sigma} .
\end{aligned}
$$


From (2.13), one finds $\left(\mathcal{J}^{++} \mathcal{J}^{-+}\right)^{\mu}{ }_{\nu}=\frac{1}{2}\left(\mathcal{J}^{++}\right)^{\mu}{ }_{\nu}$ and $\left(\mathcal{J}^{--} \mathcal{J}^{++}\right)^{\mu}{ }_{\nu}=-\frac{1}{2} \delta^{\mu}{ }_{\nu}-\left(\mathcal{J}^{+-}\right)^{\mu}{ }_{\nu}$. This implies that $K=\frac{1}{2} \chi_{\mu} \chi^{\mu}$, so $K$ is indeed the hyperkähler potential. It should be emphasized that (4.23) defines the hyperkähler potential to order $\theta^{2}$ if we impose only the auxiliary equations of motion. This is because at higher order, $\Gamma_{I}^{+}$ceases to be arctic (without imposing all the equations of motion) and so $K$ ceases to be harmonic-independent.

It will be useful to have explicit expressions for various derivatives of $K$, such as

$$
\nabla_{\underline{\alpha}}^{ \pm} K=K_{\mu} \zeta_{\alpha}^{ \pm \mu}, \quad \nabla_{b} \nabla_{\alpha}^{i} K=K_{\mu} \widehat{\nabla}_{b} \zeta_{\alpha}^{i \mu}-\Omega_{\alpha b}^{i}
$$

where $\widehat{\nabla}_{b}$ carries the target space connection.

Although we will not explicitly make use of them, it is also interesting to note that the hyperkähler one-forms $k^{i j}$ can be written in superspace as

$$
k^{++}=\Gamma_{I}^{+} \mathrm{d} \Upsilon^{I+}-\Upsilon^{I+} \mathrm{d} \Gamma_{I}^{+}, \quad \mathrm{d} k^{++}=-2 \Omega^{++} .
$$

These are given by a partial pullback of $\Omega^{++}$, replacing one d with the derivative $D^{0}$ of the auxiliary manifold. Because they are globally defined, $\Omega^{++}$is exact. Because $\Gamma_{I}^{+}$and $\Upsilon^{I+}$ are both Weyl weight one, we can rewrite this on the target space in the familiar way,

$$
\begin{aligned}
k^{++} & =\mathrm{d} \phi^{\mu} \chi^{\nu}\left(\partial_{\mu} \Upsilon^{I+} \partial_{\nu} \Gamma_{I}^{+}-\partial_{\mu} \Gamma_{I}^{+} \partial_{\nu} \Upsilon^{I+}\right)=\mathrm{d} \phi^{\mu} \Omega_{\mu \nu}^{++} \chi^{\nu} \\
& =v^{\underline{1}} v^{\underline{2}+}\left(\frac{1}{\zeta} \mathrm{d} \phi^{\mathrm{a}} \omega_{\mathrm{ab}} \chi^{\mathrm{b}}+\left(\mathrm{d} \phi^{\mathrm{a}} \chi_{\mathrm{a}}-\mathrm{d} \phi^{\overline{\mathrm{b}}} \chi_{\overline{\mathrm{b}}}\right)+\zeta \mathrm{d} \phi^{\overline{\mathrm{a}}} \omega_{\overline{\mathrm{a}} \overline{\mathrm{b}}} \chi^{\overline{\mathrm{b}}}\right) .
\end{aligned}
$$

\section{The component action in rigid projective superspace}

Now that we have established a great deal of preliminary material, we now can turn to deriving the component action from superspace. This is fairly involved, so we have chosen to separate the task into two distinct stages. In this section, we will derive the component action from rigid projective superspace. This calculation will yield a subset of the terms we actually need. Of course, the result can already be derived via a reduction to $\mathcal{N}=1$ superspace as discussed in section 2 , and we will be able to compare our result to the component version of that action. The point of this exercise is to introduce the techniques we will need in the curved case. In fact, it will turn out that reconstructing the rigid terms is actually more involved than finding the additional supergravity contributions! For this reason, we will be rather explicit in the calculation. To emphasize the applicability to the rigid case, we will avoid assuming in this section that the target space is a cone.

The action we seek to evaluate is

$$
S=-\frac{1}{2 \pi} \oint v_{i}^{+} \mathrm{d} v^{i+} \int \mathrm{d}^{4} x e \mathcal{L}^{--}, \quad \mathcal{L}^{--}=\frac{1}{16}\left(\mathcal{D}^{-}\right)^{2}\left(\overline{\mathcal{D}}^{-}\right)^{2} \mathcal{F}^{++}
$$

with $\mathcal{D}_{A}$ the gauge covariant derivative associated with rigid projective superspace. It will be convenient for later reference in the curved case to refer to $\mathcal{L}^{--}$above as $T_{0}:=$ $\frac{1}{16}\left(\mathcal{D}^{-}\right)^{2}\left(\overline{\mathcal{D}}^{-}\right)^{2} \mathcal{F}^{++}$. In the curved case, there will be additional terms. 
The function $\mathcal{F}^{++}$depends on the collective set of projective multiplets $\mathcal{Q}^{+}=$ $\left(\Upsilon^{I+}, \breve{\Upsilon}^{\bar{I}+}\right)$. We will suppress any index on $\mathcal{Q}^{+}$to keep the notation compact, denoting derivatives of the function $\mathcal{F}^{++}$as $\mathcal{F}_{\mathcal{Q}}^{+}, \mathcal{F}_{\mathcal{Q} \mathcal{Q}}$, etc. The Lagrangian $T_{0}$ can now be written

$$
\begin{aligned}
T_{0}= & \frac{1}{16}\left(\mathcal{D}^{-}\right)^{2}\left(\overline{\mathcal{D}}^{-}\right)^{2} \mathcal{Q}^{+} \mathcal{F}_{\mathcal{Q}}^{+}+\frac{1}{8} \mathcal{D}^{\alpha-} \overline{\mathcal{D}}^{\dot{\alpha}-} \mathcal{Q}^{+} \mathcal{D}_{\alpha}^{-} \overline{\mathcal{D}}_{\dot{\alpha}}^{-} \mathcal{F}_{\mathcal{Q}}^{+} \\
& +\frac{1}{8}\left(\mathcal{D}^{-}\right)^{2} \overline{\mathcal{D}}_{\dot{\alpha}}^{-} \mathcal{Q}^{+} \overline{\mathcal{D}}^{\dot{\alpha}-} \mathcal{F}_{\mathcal{Q}}^{+}+\frac{1}{8}\left(\overline{\mathcal{D}}^{-}\right)^{2} \mathcal{D}^{\alpha-} \mathcal{Q}^{+} \mathcal{D}_{\alpha}^{-} \mathcal{F}_{\mathcal{Q}}^{+} \\
& +\frac{1}{32}\left(\mathcal{D}^{-}\right)^{2} \mathcal{Q}^{+}\left(\overline{\mathcal{D}}^{-}\right)^{2} \mathcal{F}_{\mathcal{Q}}^{+}+\frac{1}{32}\left(\overline{\mathcal{D}}^{-}\right)^{2} \mathcal{Q}^{+}\left(\mathcal{D}^{-}\right)^{2} \mathcal{F}_{\mathcal{Q}}^{+} \\
& +\frac{1}{32}\left(\mathcal{D}^{-}\right)^{2} \mathcal{Q}^{+} \overline{\mathcal{D}}_{\dot{\alpha}}^{-} \mathcal{Q}^{+} \overline{\mathcal{D}}^{\dot{\alpha}-} \mathcal{Q}^{+} \mathcal{F}_{\mathcal{Q} \mathcal{Q} \mathcal{Q}}^{-}+\frac{1}{32}\left(\overline{\mathcal{D}}^{-}\right)^{2} \mathcal{Q}^{+} \mathcal{D}^{\alpha-} \mathcal{Q}^{+} \mathcal{D}_{\alpha}^{-} \mathcal{Q}^{+} \mathcal{F}_{\mathcal{Q} \mathcal{Q} \mathcal{Q}}^{-} \\
& +\frac{1}{8} \mathcal{D}^{\alpha-} \overline{\mathcal{D}}^{\dot{\alpha}-} \mathcal{Q}^{+} \mathcal{D}_{\alpha}^{-} \mathcal{Q}^{+} \overline{\mathcal{D}}_{\dot{\alpha}}^{-} \mathcal{Q}^{+} \mathcal{F}_{\mathcal{Q} \mathcal{Q} \mathcal{Q}}^{-} \\
& +\frac{1}{16} \mathcal{D}^{\alpha-} \mathcal{Q}^{+} \mathcal{D}_{\alpha}^{-} \mathcal{Q}^{+} \overline{\mathcal{D}}_{\dot{\alpha}}^{-} \mathcal{Q}^{+} \overline{\mathcal{D}}^{\dot{\alpha}-} \mathcal{Q}^{+} \mathcal{F}_{\mathcal{Q} \mathcal{Q} \mathcal{Q}}^{--}
\end{aligned}
$$

In the first three lines, we have chosen to write explicit spinor derivatives of $\mathcal{F}_{\mathcal{Q}}^{+}$rather than expanding them out. The reason is that $\mathcal{F}_{Q}^{+}=\left(i \Gamma_{I}^{+},-i \breve{\Gamma}_{\bar{I}}^{+}\right)$is arctic or antarctic to order $\theta^{2}$, and applying the auxiliary field equations tells us quite a bit about these quantities. In contrast, we cannot say anything about $\mathcal{D}_{\alpha}^{+}\left(\overline{\mathcal{D}}^{+}\right)^{2} \mathcal{F}_{\mathcal{Q}}^{+}$without applying dynamical equations of motion, so we have written $T_{0}$ in a particular way to avoid such terms.

Even in the rigid case, the expressions for the various terms we will encounter can be involved. To simplify the analysis, we will first consider only those terms that contribute in the rigid ungauged limit. Afterwards, we will include the covariant terms associated with the gauged isometries.

\subsection{Rigid ungauged terms}

Denote the first line of (5.2) by $T_{0.1}$. It can be rewritten as

$$
\begin{aligned}
T_{0.1}= & \frac{i}{16}\left(\mathcal{D}^{-}\right)^{2}\left(\overline{\mathcal{D}}^{-}\right)^{2} \Upsilon^{I+} \Gamma_{I}^{+}+\frac{i}{8} \mathcal{D}^{\alpha-} \overline{\mathcal{D}}^{\dot{\alpha}-} \Upsilon^{I+} \mathcal{D}_{\alpha}^{-} \overline{\mathcal{D}}_{\dot{\alpha}}^{-} \Gamma_{I}^{+} \\
& -\frac{i}{16}\left(\mathcal{D}^{-}\right)^{2}\left(\overline{\mathcal{D}}^{-}\right)^{2} \breve{\Upsilon}^{\bar{I}+} \breve{\Gamma}_{\bar{I}}^{+}-\frac{i}{8} \mathcal{D}^{\alpha-} \overline{\mathcal{D}}^{\dot{\alpha}-} \breve{\Upsilon}^{\bar{I}+} \mathcal{D}_{\alpha}^{-} \overline{\mathcal{D}}_{\dot{\alpha}}^{-} \breve{\Gamma}_{\bar{I}}^{+}
\end{aligned}
$$

Now let us apply the equations (4.6) for the components of the arctic multiplet. Taking only those terms that survive in the rigid ungauged limit, we find

$$
\begin{aligned}
T_{0.1} \sim & i \Gamma_{I}^{+} P^{I(-3)}+\frac{1}{2} z_{1}^{--} \Gamma_{I}^{+} \mathcal{D}^{\dot{\alpha} \alpha} \mathcal{D}_{\alpha}^{-} \overline{\mathcal{D}}_{\dot{\alpha}}^{-} \Upsilon^{I+}+\frac{i}{2}\left(z_{1}^{--}\right)^{2} \Gamma_{I}^{+} \mathcal{D}_{\alpha \dot{\alpha}} \mathcal{D}^{\dot{\alpha} \alpha} \Upsilon^{I+} \\
& -\frac{i}{8} A^{\dot{\alpha} \alpha I-} A_{\alpha \dot{\alpha}} \bar{I}+\frac{i}{4} z_{1}^{--} A^{\dot{\alpha} \alpha I-} \mathcal{D}_{\alpha \dot{\alpha}} \Gamma_{I}^{+}+\frac{i}{4} z_{1}^{--} \mathcal{D}^{\dot{\alpha} \alpha} \Upsilon^{I+} A_{\alpha \dot{\alpha}} \bar{I} \\
& -\frac{i}{2}\left(z_{1}^{--}\right)^{2} \mathcal{D}^{\dot{\alpha} \alpha} \Upsilon^{I+} \mathcal{D}_{\alpha \dot{\alpha}} \Gamma_{I}^{+}-(\text {antarctic term }) .
\end{aligned}
$$

The antarctic term is found by replacing $\Upsilon^{I+} \rightarrow \breve{\Upsilon}^{\bar{I}+}, \Gamma_{I}^{+} \rightarrow \breve{\Gamma}_{\bar{I}}^{+}$and $z_{1}^{--} \rightarrow z_{2}^{--}$. We will use the symbol $\sim$ to denote the terms we are examining at each stage of the calculation.

The above expression is valid off-shell, that is, without assuming that $\Gamma_{I}^{+}$is an arctic superfield. If we did not make this assumption, we could begin to derive it now. The 
above expression is the only place where the arctic component field $P^{I(-3)}$ appears, and so it acts as a Lagrange multiplier enforcing that the lowest component of $\Gamma_{I}^{+}$is arctic. We could proceed in this way, rederiving all of the auxiliary equations of motion, but it would be quite involved. Because we already know their content - the lower components of $\Gamma_{I}^{+}$must be arctic - it is easier to simply assume them without comment. Proceeding in this way, we see that in the expression (5.4), the terms $\Gamma_{I}^{+} P^{I(-3)}$ and $A^{\dot{\alpha} \alpha I-} A_{\alpha \dot{\alpha}} \bar{I}$ drop out under the contour integral as they are purely arctic expressions - that is, they are of the form $\frac{1}{\left(v^{1}+\right)^{2}} \sum_{n=0}^{\infty} C_{n} \zeta^{n}$ for some field-dependent coefficients $C_{n}$, and these vanish under the contour integral. Similarly, their antarctic conjugates, which take the form $\frac{1}{\left(v^{2}+\right)^{2}} \sum_{n=0}^{\infty}(-1)^{n} \bar{C}_{n} \zeta^{-n}$ also vanish. The remaining terms can be rearranged to

$$
\begin{aligned}
T_{0.1} \sim & \frac{i}{4} z_{1}^{--} \mathcal{D}^{\dot{\alpha} \alpha} \Upsilon^{I+} A_{\alpha \dot{\alpha}}--\frac{i}{4} z_{1}^{--} A^{\dot{\alpha} \alpha I-} \mathcal{D}_{\alpha \dot{\alpha}} \Gamma_{I}^{+} \\
& +\frac{1}{2} \mathcal{D}^{\dot{\alpha} \alpha}\left(z_{1}^{--} \Gamma_{I}^{+} \mathcal{D}_{\alpha}^{-} \overline{\mathcal{D}}_{\dot{\alpha}}^{-} \Upsilon^{I+}+i\left(z_{1}^{--}\right)^{2} \Gamma_{I}^{+} \mathcal{D}^{\dot{\alpha} \alpha} \Upsilon^{I+}\right)-(\text { antarctic term }) .
\end{aligned}
$$

The total derivative can be discarded in the rigid case. Now we exploit the on-shell conditions (4.10) for $A_{\alpha \dot{\alpha}}{ }^{I-}$ and $A_{\alpha \dot{\alpha}} \bar{I}$. Using the definition (3.9b) of $g_{\mathrm{ab}}$ as well as the relations

$$
z_{1}^{--}-z_{2}^{--}=\frac{1}{v \underline{1}+v^{2+}}, \quad \mathcal{D}_{\alpha \dot{\alpha}} \Upsilon^{I+}=\mathcal{D}_{\alpha \dot{\alpha}} \phi^{\mu} \partial_{\mu} \Upsilon^{I+}
$$

one finds

$$
\begin{aligned}
T_{0.1} \sim & \frac{i}{2} \frac{1}{v^{\underline{1}+} v^{\underline{2}+}} \mathcal{D}^{\dot{\alpha} \alpha} \phi^{\mathrm{a}} \mathcal{D}_{\alpha \dot{\alpha}} \phi^{\overline{\mathrm{b}}} g_{\mathrm{a} \overline{\mathrm{b}}} \\
& +\frac{1}{4} \frac{1}{v^{\underline{1}+} v^{2}+} \zeta_{\alpha}^{\mathrm{b}} \bar{\zeta}_{\dot{\alpha}}^{\overline{\mathrm{b}}} \mathcal{D}^{\dot{\alpha} \alpha} \phi^{\mu}\left(z_{1}^{--} \partial_{\mathrm{b}} \partial_{\overline{\mathrm{b}}} \Upsilon^{I+} \partial_{\mu} \Gamma_{I}^{+}-z_{1}^{--} \partial_{\mathrm{b}} \partial_{\overline{\mathrm{b}}} \Gamma_{I}^{+} \partial_{\mu} \Upsilon^{I+}-\text { a.t. }\right) .
\end{aligned}
$$

(We will occasionally abbreviate antarctic terms as "a.t.")

Next, we consider the second line of $T_{0}$. We have

$$
\begin{aligned}
& T_{0.2}=\frac{i}{8} \overline{\mathcal{D}}_{\dot{\alpha}}^{-} \Gamma_{I}^{+}\left(\mathcal{D}^{-}\right)^{2} \overline{\mathcal{D}}^{\dot{\alpha}-} \Upsilon^{I+}-\frac{i}{8} \overline{\mathcal{D}}_{\dot{\alpha}}^{-} \breve{\Gamma}_{I}^{+}\left(\mathcal{D}^{-}\right)^{2} \overline{\mathcal{D}}^{\dot{\alpha}-} \breve{\Upsilon}^{\bar{I}+}+\text { h.c. }, \\
& \sim i \Psi_{I \dot{\alpha}} \bar{\Xi}^{\dot{\alpha} I--}-\frac{z_{1}^{--}}{2} \Psi_{I \dot{\alpha}} \mathcal{D}^{\dot{\alpha} \alpha} \Psi_{\alpha}^{I}-(\text { antarctic term })+\text { h.c. }
\end{aligned}
$$

The leading term is purely arctic and vanishes under the contour integral, leaving

$$
\begin{aligned}
T_{0.2} \sim & \frac{1}{4} z_{1}^{--} \Psi_{I}^{\alpha} \overleftrightarrow{\mathcal{D}}_{\alpha \dot{\alpha}} \Psi^{I \dot{\alpha}}-\frac{1}{4} z_{1}^{--} \Psi_{I \dot{\alpha}} \overleftrightarrow{\mathcal{D}^{\dot{\alpha} \alpha}} \Psi_{\alpha}^{I} \\
& +\frac{1}{4} \mathcal{D}^{\dot{\alpha} \alpha}\left(z_{1}^{--} \mathcal{D}_{\alpha}^{-} \Gamma_{I}^{+} \overline{\mathcal{D}}_{\dot{\alpha}}^{-} \Upsilon^{I+}-z_{1}^{--} \overline{\mathcal{D}}_{\dot{\alpha}}^{-} \Gamma_{I}^{+} \mathcal{D}_{\alpha}^{-} \Upsilon^{I+}\right)-(\text { antarctic term })
\end{aligned}
$$

In the rigid case, we can discard the total derivative. Using the relations (4.9),

$$
\Psi_{I}^{\alpha} \overleftrightarrow{\mathcal{D}}_{\alpha \dot{\alpha}} \Psi^{I \dot{\alpha}}=\frac{1}{v^{\underline{1}+} v^{2}+}\left(\zeta^{\alpha \mathrm{b}} \partial_{\mathrm{b}} \Gamma_{I}^{+}\right) \overleftrightarrow{\mathcal{D}}_{\alpha \dot{\alpha}}\left(\bar{\zeta}^{\dot{\alpha} \overline{\mathrm{b}}} \partial_{\overline{\mathrm{b}}} \Upsilon^{I+}\right)
$$

which leads to

$$
\begin{aligned}
T_{0.2} \sim & \frac{1}{4} \frac{1}{v^{\underline{1}+} v^{\underline{2}+}} g_{\mathrm{b} \overline{\mathrm{b}}}\left(\zeta^{\alpha \mathrm{b}} \overleftrightarrow{\mathcal{D}}_{\alpha \dot{\alpha}} \bar{\zeta}^{\dot{\alpha} \overline{\mathrm{b}}}\right)+\frac{1}{4} \frac{1}{v^{\underline{1}+} v^{\underline{2}}+} \zeta^{\alpha \mathrm{b}} \bar{\zeta}^{\dot{\alpha} \overline{\mathrm{b}}} \mathcal{D}_{\alpha \dot{\alpha}} \phi^{\mu} \times \\
& \left(z_{1}^{--} \nabla_{\mu} \partial_{\mathrm{b}} \Gamma_{I}^{+} \partial_{\overline{\mathrm{b}}} \Upsilon^{I+}-z_{1}^{--} \nabla_{\mu} \partial_{\overline{\mathrm{b}}} \Upsilon^{I+} \partial_{\mathrm{b}} \Gamma_{I}^{+}-z_{1}^{--} \nabla_{\mu} \partial_{\mathrm{b}} \Upsilon^{I+} \partial_{\overline{\mathrm{b}}} \Gamma_{I}^{+}\right. \\
& \left.+z_{1}^{--} \nabla_{\mu} \partial_{\overline{\mathrm{b}}} \Gamma_{I}^{+} \partial_{\mathrm{b}} \Upsilon^{I+}-\text { a.t. }\right)
\end{aligned}
$$


At this stage we have recovered the kinetic terms for both scalars and fermions, along with some extra terms involving two fermions and a spacetime derivative of $\phi^{\mu}$ that should be absent in the final action. The only other contribution involving such terms is in the fifth line of $T_{0}$ :

$$
\begin{aligned}
T_{0.5}= & \frac{1}{8} \mathcal{D}^{\alpha-} \overline{\mathcal{D}}^{\dot{\alpha}-} \Upsilon^{I+} \mathcal{D}_{\alpha}^{-} \mathcal{Q}^{+} \overline{\mathcal{D}}_{\dot{\alpha}}^{-} \mathcal{Q}^{+} \mathcal{F}_{I \mathcal{Q} \mathcal{Q}}^{-}+\text {a.t. } \\
\sim & \frac{i}{4}\left(\frac{1}{v^{\underline{1}+} v^{\underline{2}+}} \mathcal{D}^{\dot{\alpha} \alpha} \bar{\phi}^{\overline{\mathrm{b}}} \partial_{\overline{\mathrm{b}}} \Upsilon^{I+}-z_{1}^{--} \mathcal{D}^{\alpha \dot{\alpha}} \Upsilon^{I+}\right) \mathcal{D}_{\alpha}^{-} \mathcal{Q}^{+} \overline{\mathcal{D}}_{\dot{\alpha}}^{-} \mathcal{Q}^{+} \mathcal{F}_{I \mathcal{Q} \mathcal{Q}}^{-} \\
& -\frac{i}{4}\left(\frac{1}{v^{\underline{1}+} v^{\underline{2}+}} \mathcal{D}^{\dot{\alpha} \alpha} \phi^{\mathrm{b}} \partial_{\mathrm{b}} \breve{\Upsilon}^{\bar{I}+}+z_{2}^{--} \mathcal{D}^{\alpha \dot{\alpha}} \breve{\Upsilon}^{\bar{I}+}\right) \mathcal{D}_{\alpha}^{-} \mathcal{Q}^{+} \overline{\mathcal{D}}_{\dot{\alpha}}^{-} \mathcal{Q}^{+} \mathcal{F}_{\bar{I} \mathcal{Q} \mathcal{Q}}^{-} \\
& -\frac{1}{8} \frac{1}{v^{\underline{1}+} v^{2+}} \zeta^{\alpha \mathrm{b}} \bar{\zeta}^{\dot{\alpha} \bar{b}} \partial_{\mathrm{b}} \partial_{\overline{\mathrm{b}}} \mathcal{Q}^{+} \mathcal{D}_{\alpha}^{-} \mathcal{Q}^{+} \overline{\mathcal{D}}_{\dot{\alpha}}^{-} \mathcal{Q}^{+} \mathcal{F}_{\mathcal{Q} \mathcal{Q} \mathcal{Q}}^{-}
\end{aligned}
$$

The terms in question can be written as

$$
T_{0.1}+T_{0.2}+T_{0.5} \sim \frac{1}{4 v^{1}+v^{2}+} \zeta^{\alpha \mathrm{b}} \bar{\zeta}^{\dot{\alpha} \overline{\mathrm{b}}} \mathcal{D}_{\alpha \dot{\alpha}} \phi^{\mathrm{c}} \mathcal{S}_{\mathrm{cb} \overline{\mathrm{b}}}+\text { h.c. }
$$

where $\mathcal{S}_{\mathrm{cb} \overline{\mathrm{b}}}$ is given by

$$
\begin{aligned}
\mathcal{S}_{\mathrm{cb} \overline{\mathrm{b}}}= & z_{1}^{--}\left(\nabla_{\mathrm{c}} \partial_{\mathrm{b}} \Gamma_{I}^{+} \partial_{\overline{\mathrm{b}}} \Upsilon^{I+}-\nabla_{\mathrm{c}} \partial_{\mathrm{b}} \Upsilon^{I+} \partial_{\overline{\mathrm{b}}} \Gamma_{I}^{+}\right)+2 z_{1}^{--} \nabla_{[\mathrm{c}}\left(\partial_{\mathrm{b}]} \Upsilon^{I+} \partial_{\overline{\mathrm{b}}} \Gamma_{I}^{+}-\partial_{\mathrm{b}]} \Gamma_{I}^{+} \partial_{\overline{\mathrm{b}}} \Upsilon^{I+}\right) \\
& -z_{2}^{--}\left(\nabla_{\mathrm{c}} \partial_{\mathrm{b}} \breve{\Gamma}_{\bar{I}}^{+} \partial_{\overline{\mathrm{b}}} \breve{\Upsilon}^{\bar{I}+}-\nabla_{\mathrm{c}} \partial_{\mathrm{b}} \breve{\Upsilon}^{\bar{I}+} \partial_{\overline{\mathrm{b}}} \breve{\Gamma}_{\bar{I}}^{+}\right)-2 z_{2}^{--} \nabla_{[\mathrm{c}}\left(\partial_{\mathrm{b}]} \breve{\Upsilon}^{\bar{I}+} \partial_{\overline{\mathrm{b}}} \breve{\Gamma}_{\bar{I}}^{+}-\partial_{\mathrm{b}]} \breve{\Gamma}_{\bar{I}}^{+} \partial_{\overline{\mathrm{b}}} \breve{\Upsilon}^{\bar{I}+}\right) \\
& +i z_{1}^{--} \partial_{\mathrm{c}} \mathcal{Q}^{+} \partial_{\mathrm{b}} \mathcal{Q}^{+} \partial_{\overline{\mathrm{b}}} \mathcal{Q}^{+} \mathcal{F}_{\mathcal{Q} \mathcal{Q}}^{-}
\end{aligned}
$$

We want to show that this expression vanishes. The second and fourth terms of $\mathcal{S}_{\mathrm{cb} \bar{b}}$ are proportional to $\nabla_{[\mathrm{c}} g_{\mathrm{b}] \overline{\mathrm{b}}}$, which vanishes. With the useful identity

$$
\begin{aligned}
\partial_{\mu} \mathcal{Q}^{+} \partial_{\nu} \mathcal{Q}^{+} \partial_{\rho} \mathcal{Q}^{+} \mathcal{F}_{\mathcal{Q} \mathcal{Q} \mathcal{Q}}^{-}= & -i \partial_{\mu} \partial_{\nu} \Upsilon^{I+} \partial_{\rho} \Gamma_{I}^{+}+i \partial_{\rho} \Upsilon^{I+} \partial_{\mu} \partial_{\nu} \Gamma_{I}^{+} \\
& +i \partial_{\mu} \partial_{\nu} \breve{\Upsilon}^{\bar{I}+} \partial_{\rho} \breve{\Gamma}_{\bar{I}}^{+}-i \partial_{\rho} \breve{\Upsilon}^{\bar{I}+} \partial_{\mu} \partial_{\nu} \breve{\Gamma}_{\bar{I}}^{+}
\end{aligned}
$$

the remaining terms can be rewritten as

$$
\mathcal{S}_{\mathrm{cb} \overline{\mathrm{b}}}=\frac{1}{v^{1}+v^{2}+}\left[\nabla_{\mathrm{c}}\left(\partial_{\mathrm{b}} \breve{\Gamma}_{\bar{I}}^{+} \partial_{\overline{\mathrm{b}}} \breve{\Upsilon}^{\bar{I}+}-\partial_{\mathrm{b}} \breve{\Upsilon}^{\bar{I}+} \partial_{\overline{\mathrm{b}}} \breve{\Gamma}_{\bar{I}}^{+}\right)-\left(\partial_{\mathrm{b}} \breve{\Gamma}_{\bar{I}}^{+} \partial_{\overline{\mathrm{b}}} \partial_{\mathrm{c}} \breve{\Upsilon}^{\bar{I}+}-\partial_{\mathrm{b}} \breve{\Upsilon}^{\bar{I}+} \partial_{\overline{\mathrm{b}}} \partial_{\mathrm{c}} \breve{\Gamma}_{\bar{I}}^{+}\right)\right]
$$

The first set of terms involves $\nabla_{\mathrm{c}} g_{\mathrm{b} \overline{\mathrm{b}}}$, which vanishes. The second set is purely antarctic and so vanishes under the contour integral. Thus $\mathcal{S}_{\mathrm{cb} \bar{b}}$ does indeed drop out. This leaves

$$
\begin{aligned}
T_{0.1}+T_{0.2}+T_{0.5} \sim & \frac{i}{2} \frac{1}{v^{\underline{1}+} v^{\underline{2}+}} \mathcal{D}^{\dot{\alpha} \alpha} \phi^{\mathrm{a}} \mathcal{D}_{\alpha \dot{\alpha}} \phi^{\overline{\mathrm{b}}} g_{\mathrm{a} \overline{\mathrm{b}}}+\frac{1}{4} \frac{1}{v^{\underline{1}+} v^{2+}} g_{\mathrm{b} \overline{\mathrm{b}}}\left(\zeta^{\alpha \mathrm{b}} \overleftrightarrow{\widehat{\mathcal{D}}}_{\alpha \dot{\alpha}} \bar{\zeta}^{\dot{\alpha} \overline{\mathrm{b}}}\right) \\
& +\frac{1}{8} \frac{1}{\left(v^{\underline{1}+} v^{2}+\right)^{2}} \zeta^{\mathrm{a}} \zeta^{\mathrm{b}} \bar{\zeta}^{\overline{\mathrm{a}}} \bar{\zeta}^{\overline{\mathrm{b}}} \partial_{\mathrm{a}} \partial_{\overline{\mathrm{a}}} \mathcal{Q}^{+} \partial_{\mathrm{b}} \mathcal{Q}^{+} \partial_{\overline{\mathrm{b}}} \mathcal{Q}^{+} \mathcal{F}_{\mathcal{Q} \mathcal{Q}}^{-}
\end{aligned}
$$

The only term we must still reconstruct is the four fermion term involving the hyperkähler curvature. It should be found by including the remaining terms in the third, 
fourth and sixth lines of $T_{0}$. The third line is purely arctic or antarctic in the absence of gauged isometries so we may ignore it. The fourth and sixth lines give

$$
\begin{aligned}
& T_{0.4}=\frac{1}{32}\left(\mathcal{D}^{-}\right)^{2} \Upsilon^{I+} \overline{\mathcal{D}}_{\dot{\alpha}}^{-} \mathcal{Q}^{+} \overline{\mathcal{D}}^{\dot{\alpha}-} \mathcal{Q}^{+} \mathcal{F}_{I \mathcal{Q Q}}^{-}+\frac{1}{32}\left(\overline{\mathcal{D}}^{-}\right)^{2} \Upsilon^{I+} \mathcal{D}^{\alpha-} \mathcal{Q}^{+} \mathcal{D}_{\alpha}^{-} \mathcal{Q}^{+} \mathcal{F}_{I \mathcal{Q Q}}^{-}+\text {a.t. } \\
& \sim \frac{1}{32} \frac{\zeta^{\mathrm{a}} \zeta^{\mathrm{b}} \bar{\zeta}^{\overline{\mathrm{a}}} \bar{\zeta}^{\overline{\mathrm{b}}}}{\left(v^{\underline{1}+} v^{2}\right)^{2}}\left(\nabla_{\mathrm{a}} \partial_{\mathrm{b}} \mathcal{Q}^{+} \partial_{\overline{\mathrm{a}}} \mathcal{Q}^{+} \partial_{\overline{\mathrm{b}}} \mathcal{Q}^{+}+\nabla_{\overline{\mathrm{a}}} \partial_{\overline{\mathrm{b}}} \mathcal{Q}^{+} \partial_{\mathrm{a}} \mathcal{Q}^{+} \partial_{\mathrm{b}} \mathcal{Q}^{+}\right) \mathcal{F}_{\mathcal{Q} \mathcal{Q} \mathcal{Q}}^{-} \\
& T_{0.6} \sim \frac{1}{16} \frac{1}{\left(v^{1}+v^{2}+\right)^{2}} \zeta^{\mathrm{a}} \zeta^{\mathrm{b}} \bar{\zeta}^{\overline{\mathrm{a}}} \bar{\zeta}^{\overline{\mathrm{b}}}\left(\partial_{\mathrm{a}} \mathcal{Q}^{+} \partial_{\mathrm{b}} \mathcal{Q}^{+} \partial_{\overline{\mathrm{b}}} \mathcal{Q}^{+} \partial_{\overline{\mathrm{b}}} \mathcal{Q}^{+} \mathcal{F}_{\mathcal{Q} \mathcal{Q} \mathcal{Q}}^{--}\right)
\end{aligned}
$$

Combining all terms, we have

$$
T_{0} \sim \frac{i}{v^{\underline{1}+} v^{\underline{2}+}}\left[\frac{1}{2} \mathcal{D}^{\dot{\alpha} \alpha} \phi^{\mathrm{a}} \mathcal{D}_{\alpha \dot{\alpha}} \phi^{\overline{\mathrm{b}}} g_{\mathrm{a} \overline{\mathrm{b}}}-\frac{i}{4} g_{\mathrm{b} \overline{\mathrm{b}}}\left(\zeta^{\alpha \mathrm{b}} \overleftrightarrow{\mathcal{D}}_{\alpha \dot{\alpha}} \bar{\zeta}^{\dot{\alpha} \overline{\mathrm{b}}}\right)\right]+\frac{1}{16} \frac{1}{\left(v^{\underline{1}+} v^{2+}\right)^{2}} \zeta^{\mathrm{a}} \zeta^{\mathrm{b}} \bar{\zeta}^{\overline{\mathrm{a}}} \bar{\zeta}^{\overline{\mathrm{b}}} \mathcal{R}_{\mathrm{ab} \overline{\mathrm{a}} \overline{\mathrm{b}}}^{++}
$$

where

$$
\begin{aligned}
\mathcal{R}_{\mathrm{ab} \overline{\mathrm{b}} \mathrm{b}}^{++} & =\frac{1}{2} \nabla_{\overline{\mathrm{a}}} \Delta_{\overline{\mathrm{b}} \mathrm{b}}^{++}+\text {h.c. } \\
\Delta_{\overline{\mathrm{a}} \mathrm{b}}^{++} & :=\partial_{\overline{\mathrm{a}}} \mathcal{Q}^{+} \partial_{\mathrm{b}} \mathcal{Q}^{+} \partial_{\mathrm{c}} \mathcal{Q}^{+} \mathcal{F}_{\mathcal{Q} \mathcal{Q} \mathcal{Q}}^{-} \\
& =-i \nabla_{\mathrm{b}} \partial_{\mathrm{c}} \Upsilon^{I+} \partial_{\overline{\mathrm{a}}} \Gamma_{I}^{+}+i \nabla_{\mathrm{b}} \partial_{\mathrm{c}} \Gamma_{I}^{+} \partial_{\overline{\mathrm{a}}} \Upsilon^{I+}+i \partial_{\overline{\mathrm{a}}} \partial_{\mathrm{b}} \breve{\Upsilon}^{\bar{I}+} \partial_{\mathrm{c}} \breve{\Gamma}_{\bar{I}}^{+}-i \partial_{\overline{\mathrm{a}}} \partial_{\mathrm{b}} \breve{\Gamma}_{\bar{I}}^{+} \partial_{\mathrm{c}} \breve{\Upsilon}^{\bar{I}+}
\end{aligned}
$$

The contribution to $\Delta_{\overline{\mathrm{b}} \mathrm{ab}}^{++}$from the antarctic fields is purely antarctic when divided by $\left(v^{\underline{1}} v^{\underline{2}+}\right)^{2}$, so it drops out. The remaining terms contribute to $R_{\text {abā̄ }}^{++}$as

$$
\begin{aligned}
\mathcal{R}_{\mathrm{ab} \overline{\mathrm{a}} \overline{\mathrm{b}}}^{++} \sim & -i \nabla_{\overline{\mathrm{a}}} \nabla_{\mathrm{b}} \partial_{\mathrm{a}} \Upsilon^{I+} \partial_{\overline{\mathrm{b}}} \Gamma_{I}^{+}+i \nabla_{\overline{\mathrm{a}}} \nabla_{\mathrm{b}} \partial_{\mathrm{a}} \Gamma_{I}^{+} \partial_{\overline{\mathrm{b}}} \Upsilon^{I+} \\
& -i \nabla_{\mathrm{b}} \partial_{\mathrm{a}} \Upsilon^{I+} \nabla_{\overline{\mathrm{a}}} \partial_{\overline{\mathrm{b}}} \Gamma_{I}^{+}+i \nabla_{\mathrm{b}} \partial_{\mathrm{a}} \Gamma_{I}^{+} \nabla_{\overline{\mathrm{a}}} \partial_{\overline{\mathrm{b}}} \Upsilon^{I+}+\text { h.c. }
\end{aligned}
$$

The second line vanishes under the contour integral since

$$
\nabla_{\overline{\mathrm{a}}} \partial_{\overline{\mathrm{b}}} \Upsilon^{I+}=\left(\frac{v^{\underline{2}+}}{v^{\underline{1}}}\right)^{2} \omega_{\overline{\mathrm{b}}}^{\mathrm{b}} \omega_{\overline{\mathrm{a}}}^{\mathrm{a}} \nabla_{\mathrm{a}} \partial_{\mathrm{b}} \Upsilon^{I+}
$$

is purely arctic when divided by $\left(v^{\underline{1}+} v^{\underline{2}^{2}}\right)^{2}$. The first line simplifies in the same way after commuting $\nabla_{\bar{a}}$ to act on the arctic superfields. This leaves

$$
\begin{aligned}
\mathcal{R}_{\mathrm{ab} \overline{\mathrm{a}} \mathrm{b}}^{++} & \sim-i\left[\nabla_{\overline{\mathrm{a}}}, \nabla_{\mathrm{b}}\right] \partial_{\mathrm{a}} \Upsilon^{I+} \partial_{\overline{\mathrm{b}}} \Gamma_{I}^{+}+i\left[\nabla_{\overline{\mathrm{a}}}, \nabla_{\mathrm{b}}\right] \partial_{\mathrm{a}} \Gamma_{I}^{+} \partial_{\overline{\mathrm{b}}} \Upsilon^{I+}+\text { h.c. } \\
& =i R_{\mathrm{b} \overline{\mathrm{a}}} \mathrm{c}\left(\partial_{\mathrm{c}} \Upsilon^{I+} \partial_{\overline{\mathrm{b}}} \Gamma_{I}^{+}-\partial_{\mathrm{c}} \Gamma_{I}^{+} \partial_{\overline{\mathrm{b}}} \Upsilon^{I+}\right)=i v^{\underline{1}} v^{\underline{2}} R_{\mathrm{a} \overline{\mathrm{a}} \overline{\mathrm{b}}}
\end{aligned}
$$

The final result for the rigid ungauged contributes to $T_{0}$, including the previously discarded total covariant derivatives, is

$$
\begin{aligned}
T_{0} \sim & \frac{i}{v^{\underline{1}+} v^{2}+}\left(\frac{1}{2} \mathcal{D}^{\dot{\alpha} \alpha} \phi^{\mathrm{a}} \mathcal{D}_{\alpha \dot{\alpha}} \phi^{\overline{\mathrm{b}}} g_{\mathrm{a} \overline{\mathrm{b}}}-\frac{i}{4} g_{\mathrm{b} \overline{\mathrm{b}}}\left(\zeta^{\mathrm{\alpha}} \overleftrightarrow{\widehat{\mathcal{D}}}_{\alpha \dot{\alpha}} \bar{\zeta}^{\dot{\alpha} \overline{\mathrm{b}}}\right)+\frac{1}{16} \zeta^{\mathrm{a}} \zeta^{\mathrm{b}} \bar{\zeta}^{\overline{\mathrm{a}}} \bar{\zeta}^{\overline{\mathrm{b}}} R_{\mathrm{a} \overline{\mathrm{a}} \overline{\mathrm{b}}}\right) \\
& +\mathcal{D}^{\dot{\alpha} \alpha}\left(\frac{1}{2} z_{1}^{--} \Gamma_{I}^{+} \mathcal{D}_{\alpha}^{-} \overline{\mathcal{D}}_{\dot{\alpha}}^{-} \Upsilon^{I+}+\frac{i}{2}\left(z_{1}^{--}\right)^{2} \Gamma_{I}^{+} \mathcal{D}^{\dot{\alpha} \alpha} \Upsilon^{I+}\right. \\
& \left.+\frac{1}{4} z_{1}^{--} \mathcal{D}_{\alpha}^{-} \Gamma_{I}^{+} \overline{\mathcal{D}}_{\dot{\alpha}}^{-} \Upsilon^{I+}-\frac{1}{4} z_{1}^{--} \overline{\mathcal{D}}_{\dot{\alpha}}^{-} \Gamma_{I}^{+} \mathcal{D}_{\alpha}^{-} \Upsilon^{I+}-\text { a.t. }\right) .
\end{aligned}
$$


We will return to this expression later when we address the supergravity contributions. Now let us remain with the rigid case and simplify. The contour integral is completely trivial since only the prefactor varies along $\mathcal{C}$,

$$
-\frac{1}{2 \pi} \oint_{\mathcal{C}} v_{i}^{+} \mathrm{d} v^{i+} \frac{i}{v^{\underline{1}+} v^{2}+}=\oint_{\mathcal{C}} \frac{\mathrm{d} \zeta}{2 \pi i \zeta}=1
$$

This leads (dropping the total covariant derivative) to

$$
\mathcal{L}=\frac{1}{2} \mathcal{D}^{\dot{\alpha} \alpha} \phi^{\mathrm{a}} \mathcal{D}_{\alpha \dot{\alpha}} \phi^{\overline{\mathrm{b}}} g_{\mathrm{a} \overline{\mathrm{b}}}-\frac{i}{4} g_{\mathrm{b} \overline{\mathrm{b}}}\left(\zeta^{\alpha \mathrm{b}} \overleftrightarrow{\mathcal{D}}_{\alpha \dot{\alpha}} \bar{\zeta}^{\dot{\alpha} \overline{\mathrm{b}}}\right)+\frac{1}{16} \zeta^{\mathrm{a}} \zeta^{\mathrm{b}} \bar{\zeta}^{\overline{\mathrm{a}}} \bar{\zeta}^{\overline{\mathrm{b}}} R_{\mathrm{a} \overline{\mathrm{a}} \mathrm{b} \overline{\mathrm{b}}}
$$

for the component Lagrangian. This is the general form of the hypermultiplet action in holomorphic coordinates $\phi^{\mathrm{a}}$ in a rigid background in the absence of gauged isometries.

\subsection{Rigid gauged terms}

Our next step is to collect the terms corresponding to gauged isometries. We return to the expression (5.2) and collect the terms involving the scalar field $W^{r}$, the gaugino $\lambda_{\alpha i}^{r}$ and the auxiliary field $Y^{r i j}$ of the vector multiplet.

Let us begin with terms involving $Y^{\text {rij }}$. These come only from the first term of $T_{0}$, which gives

$$
\begin{aligned}
T_{0} \sim & 3 i z_{1}^{--} Y^{r--} \mathcal{J}_{r}^{I+} \Gamma_{I}^{+}-3 i\left(z_{1}^{--}\right)^{2} Y^{r-+} \mathcal{J}_{r}^{I+} \Gamma_{I}^{+}+i\left(z_{1}^{--}\right)^{3} Y^{r++} \mathcal{J}_{r}^{I+} \Gamma_{I}^{+}-\text {a.t. } \\
\sim & i\left(z_{1}^{--}-z_{2}^{--}\right)\left[Y^{r--} D_{r}^{++}-2 Y^{r-+} D_{r}^{+-}+Y^{r++} D_{r}^{--}\right] \\
& +D^{--}\left[i\left(z_{1}^{--}-z_{2}^{--}\right)\left(2 Y^{r+-} D_{r}^{++}-Y^{r++} D_{r}^{+-}-\frac{1}{2}\left(z_{1}^{--}+z_{2}^{--}\right) Y^{r++} D_{r}^{++}\right)\right]
\end{aligned}
$$

after identifying $\mathcal{J}_{r}^{I+} \Gamma_{I}^{+} \equiv D_{r}^{++}$as the $\mathcal{N}=2$ moment map. The argument of $D^{--}$is holomorphic away from the poles, so it can be discarded, leaving

$$
T_{0} \sim \frac{i}{v \underline{1}+v^{2}+} Y_{i j}^{r} D_{r}^{i j}
$$

Now let's collect all terms involving the chiral gaugino $\lambda_{\alpha i}^{r}$. These are

$$
\begin{aligned}
T_{0} \sim & i \Gamma_{I}^{+}\left(2 z_{1}^{--} \lambda^{\alpha r-} \mathcal{D}_{\alpha}^{-} \mathcal{J}_{r}^{I+}-\left(z_{1}^{--}\right)^{2} \lambda^{\alpha r+} \mathcal{D}_{\alpha}^{-} \mathcal{J}_{r}^{I+}\right) \\
& +i \mathcal{D}^{\alpha-} \Gamma_{I}^{+}\left(2 z_{1}^{--} \lambda_{\alpha}^{r-} \mathcal{J}_{r}^{I+}-\left(z_{1}^{--}\right)^{2} \lambda_{\alpha}^{r+} \mathcal{J}_{r}^{I+}\right)-(\operatorname{antarctic} \text { term }) \\
= & i\left(z_{1}^{--}-z_{2}^{--}\right) \lambda^{\alpha r-} \mathcal{D}_{\alpha}^{-} D_{r}^{++}-i\left(z_{1}^{--}-z_{2}^{--}\right) \lambda^{\alpha r+} D^{--} \mathcal{D}_{\alpha}^{-} D_{r}^{++} \\
& +i D^{--}\left[\left(z_{1}^{--}-z_{2}^{--}\right) \lambda^{\alpha r+} \mathcal{D}_{\alpha}^{-} D_{r}^{++}\right] .
\end{aligned}
$$

Recalling that $\mathcal{D}_{\alpha}^{-} D_{r}^{++}=\Omega_{r \alpha}^{+}=v_{i}^{+} \Omega_{r \alpha}^{i}$ is holomorphic, the argument of $D^{--}$is clearly holomorphic so it can be discarded. The remaining terms can be rewritten as

$$
T_{0} \sim \frac{i}{v^{\underline{1}}+v^{\underline{2}}+} \lambda_{i}^{\alpha r} \Omega_{r \alpha}^{i}
$$


Now let's address all terms involving $W^{r}$ or its conjugate. Those from the first two lines of $T_{0}$ give (after some algebra)

$$
\begin{aligned}
T_{0.1}+T_{0.2} \sim & \frac{i}{12} z_{1}^{--}\left(\bar{W}^{s} \overline{\mathcal{D}}_{i j} D_{s}^{i j}+\text { h.c. }\right)-\frac{i}{4} z_{1}^{--} \bar{W}^{s}\left(\overline{\mathcal{D}}^{-}\right)^{2} \Gamma_{I}^{+} \mathcal{J}_{s}^{I+}-\frac{i}{4} z_{1}^{--} W^{r}\left(\mathcal{D}^{-}\right)^{2} \Gamma_{I}^{+} \mathcal{J}_{r}^{I+} \\
& -\frac{i}{2}\left(z_{1}^{--}\right)^{2} W^{r} \bar{W}^{s} \Gamma_{I}^{+}\left\{X_{r}, X_{s}\right\} \Upsilon^{I+}-(\text { antarctic term })
\end{aligned}
$$

Now we combine these with those terms from $T_{0.3}$, which can be rewritten as

$$
\begin{aligned}
T_{0.3} \sim & \frac{i}{2}\left(M_{I}^{-}-z_{1}^{--} \bar{W}^{s} \mathcal{J}_{s I}^{+}\right)\left(N^{I-}-z_{1}^{--} W^{r} \mathcal{J}_{r}^{I+}\right) \\
& +\frac{i}{2}\left(N_{I}^{-}-z_{1}^{--} W^{r} \mathcal{J}_{r I}^{+}\right)\left(M^{I-}-z_{1}^{--} \bar{W}^{s} \mathcal{J}_{s}^{I+}\right)-(\text { antarctic term })
\end{aligned}
$$

The terms in $T_{0.3}$ independent of $z_{1}^{--}$vanish under the contour integral as they are purely arctic. Combining all the other terms gives

$$
\begin{aligned}
T_{0.1}+T_{0.2}+T_{0.3} \sim & \frac{i}{2} \frac{1}{v^{1+} v^{\underline{2}+}} W^{r} \bar{W}^{s} J_{r}^{\mu} J_{s}^{\nu} g_{\mu \nu}+\frac{i}{12} \frac{1}{v^{1+} v^{\underline{2}}+}\left(\bar{W}^{s} \overline{\mathcal{D}}_{i j} D_{s}^{i j}+\text { h.c. }\right) \\
& +\left[\frac{i}{8} \frac{z_{1}^{--}}{v^{\underline{1}+} v^{\underline{2}}+} W^{r} J_{r}^{\mu} \zeta^{\mathrm{a}} \zeta^{\mathrm{b}} \omega_{\mathrm{b}} \overline{\mathrm{b}}\left(\partial_{\mu} \Upsilon^{I+} \partial_{\mathrm{a}} \partial_{\overline{\mathrm{b}}} \Gamma_{I}^{+}-\partial_{\mu} \Gamma_{I}^{+} \partial_{\mathrm{a}} \partial_{\overline{\mathrm{b}}} \Upsilon^{I+}\right)-\text { a.t. }\right] \\
& -\left[\frac{i}{8} \frac{z_{1}^{--}}{v^{\underline{1}+} v^{\underline{2}}+} \bar{W}^{r} J_{r}^{\mu} \bar{\zeta}^{\overline{\mathrm{a}}} \bar{\zeta}^{\overline{\mathrm{b}}} \omega_{\overline{\mathrm{b}}}^{\mathrm{b}}\left(\partial_{\mu} \Upsilon^{I+} \partial_{\overline{\mathrm{a}}} \partial_{\mathrm{b}} \Gamma_{I}^{+}-\partial_{\mu} \Gamma_{I}^{+} \partial_{\overline{\mathrm{a}}} \partial_{\mathrm{b}} \Upsilon^{I+}\right)-\text { a.t. }\right] .
\end{aligned}
$$

The only other term containing explicit $W^{r}$ or $\bar{W}^{r}$ contributions comes from $T_{0.4}$ :

$$
\begin{aligned}
T_{0.4} \sim & \frac{1}{8} \frac{1}{v^{\underline{1}+} v^{\underline{2}+}} \bar{W}^{s} J_{s}^{\mu} \bar{\zeta}^{\overline{\mathrm{a}}} \bar{\zeta}^{\overline{\mathrm{b}}} \omega_{\overline{\mathrm{b}}}^{\mathrm{b}}\left(z_{1}^{--} \partial_{\mu} \Upsilon^{I+} \partial_{\overline{\mathrm{a}}} \mathcal{Q}^{+} \partial_{\mathrm{b}} \mathcal{Q}^{+} \mathcal{F}_{I \mathcal{Q}}^{-}+\text {a.t. }\right) \\
& -\frac{1}{8} \frac{1}{\left(v^{\underline{1}+} v^{2+}\right)^{2}} \bar{W}^{s} J_{s}^{\overline{\mathrm{c}}} \bar{\zeta}^{\overline{\mathrm{a}}} \bar{\zeta}^{\overline{\mathrm{b}}} \omega_{\overline{\mathrm{b}}}^{\mathrm{b}}\left(\partial_{\overline{\mathrm{c}}} \Upsilon^{I+} \partial_{\overline{\mathrm{a}}} \mathcal{Q}^{+} \partial_{\mathrm{b}} \mathcal{Q}^{+} \mathcal{F}_{I \mathcal{Q} \mathcal{Q}}^{-}\right) \\
& +\frac{1}{8} \frac{1}{\left(v^{\underline{1}+} v^{\underline{2}+}\right)^{2}} \bar{W}^{s} J_{s}^{\mathrm{c}} \bar{\zeta}^{\overline{\mathrm{c}}} \bar{\zeta}^{\overline{\mathrm{b}}} \omega_{\overline{\mathrm{b}}}^{\mathrm{b}}\left(\partial_{\mathrm{c}} \breve{\Upsilon}^{\bar{I}+} \partial_{\overline{\mathrm{a}}} \mathcal{Q}^{+} \partial_{\mathrm{b}} \mathcal{Q}^{+} \mathcal{F}_{\bar{I} \mathcal{Q}}^{-}\right)+\text {h.c. }
\end{aligned}
$$

Combining all terms that go as $W^{r} \zeta^{\mathrm{a}} \zeta^{\mathrm{b}}$, we find (after some simplifications)

$$
\begin{aligned}
& \frac{1}{8} \frac{1}{\left(v^{\underline{1}+} v^{2}+\right)^{2}} W^{r} J_{r}^{\mathrm{c}} \zeta^{\mathrm{a}} \zeta^{\mathrm{b}} \omega_{\mathrm{b}} \overline{\mathrm{b}}\left(i \partial_{\mathrm{c}} \breve{\Upsilon}^{\bar{I}+} \partial_{\mathrm{a}} \partial_{\overline{\mathrm{b}}} \breve{\Gamma}_{\bar{I}}^{+}-i \partial_{\mathrm{c}} \breve{\Gamma}_{\bar{I}}^{+} \partial_{\mathrm{a}} \partial_{\overline{\mathrm{b}}} \breve{\Upsilon}^{\bar{I}+}\right) \\
& +\frac{1}{8} \frac{1}{\left(v^{\underline{1}+} v^{2+}\right)^{2}} W^{r} J_{r}^{\overline{\mathrm{c}}} \zeta^{\mathrm{a}} \zeta^{\mathrm{b}} \omega_{\mathrm{b}} \overline{\mathrm{b}}\left(i \partial_{\overline{\mathrm{c}}} \Upsilon^{I+} \partial_{\mathrm{a}} \partial_{\overline{\mathrm{b}}} \Gamma_{I}^{+}-i \partial_{\overline{\mathrm{c}}} \Gamma_{I}^{+} \partial_{\mathrm{a}} \partial_{\overline{\mathrm{b}}} \Upsilon^{I+}\right)
\end{aligned}
$$

The first line is purely antarctic and the second is arctic, so these drop out.

The full contribution from gauged isometries yields

$$
T_{0} \sim \frac{i}{v^{\underline{1}+} v^{\underline{2}+}}\left(Y_{i j}^{r} D_{r}^{i j}+\frac{1}{2} W^{r} \bar{W}^{s} J_{r}^{\mu} J_{s}^{\nu} g_{\mu \nu}+\left(\lambda_{i}^{\alpha r} \Omega_{r \alpha}^{i}+\frac{1}{12} W^{r} \mathcal{D}_{i j} D_{r}^{i j}+\text { h.c. }\right)\right) .
$$

Using the explicit relation (4.22) for $\mathcal{D}_{i j} D_{r}^{i j}$, performing the contour integral, and combining with the result (5.27) of the previous section, we find the full rigid Lagrangian

$$
\begin{aligned}
\mathcal{L}= & \frac{1}{2} \mathcal{D}^{\dot{\alpha} \alpha} \phi^{\mathrm{a}} \mathcal{D}_{\alpha \dot{\alpha}} \phi^{\overline{\mathrm{b}}} g_{\mathrm{a} \overline{\mathrm{b}}}-\frac{i}{4} g_{\mathrm{b} \overline{\mathrm{b}}}\left(\zeta^{\alpha \mathrm{b}} \overleftrightarrow{\mathcal{D}}_{\alpha \dot{\alpha}} \bar{\zeta}^{\dot{\mathrm{d}} \overline{\mathrm{b}}}\right)+\frac{1}{16} \zeta^{\mathrm{a}} \zeta^{\mathrm{b}} \bar{\zeta}^{\overline{\mathrm{a}}} \bar{\zeta}^{\overline{\mathrm{b}}} R_{\mathrm{a} \overline{\mathrm{a}} \mathrm{b} \overline{\mathrm{b}}} \\
& +Y_{i j}^{r} D_{r}^{i j}+\left(\lambda_{i}^{\alpha r} \Omega_{r \alpha}^{i}+\text { h.c. }\right)-\frac{1}{2} W^{r} \bar{W}^{s} J_{r}^{\mu} J_{s}^{\nu} g_{\mu \nu} \\
& -\frac{1}{4} W^{r} \zeta^{\mathrm{a}} \zeta^{\mathrm{b}} \nabla_{\mathrm{a}}\left(\omega_{\mathrm{bc}} J_{r}^{\mathrm{c}}\right)-\frac{1}{4} \bar{W}^{r} \bar{\zeta}^{\overline{\mathrm{a}}} \bar{\zeta}^{\overline{\mathrm{b}}} \nabla_{\mathrm{a}}\left(\omega_{\overline{\mathrm{b}} \overline{\mathrm{c}}} J_{r}^{\overline{\mathrm{c}}}\right) .
\end{aligned}
$$


This coincides with the component version of the $\mathcal{N}=1$ action [59]

$$
S=\int \mathrm{d}^{4} x \mathrm{~d}^{4} \theta K-\int \mathrm{d}^{4} x \mathrm{~d}^{2} \theta W^{r} \Lambda_{r}-\int \mathrm{d}^{4} x \mathrm{~d}^{2} \bar{\theta} \bar{W}^{r} \bar{\Lambda}_{r}
$$

upon eliminating the auxiliary fields associated with the $\mathcal{N}=1$ chiral multiplets $\phi^{\text {a }}{ }^{19}$ The superpotential contribution couples the chiral superfield $W^{r}$ of the $\mathcal{N}=2$ vector multiplet to the chiral component $\Lambda_{r}$ of the $\mathcal{N}=2$ moment map. The remaining component of the moment map is contained implicitly within the Kähler potential.

\section{The component action in curved projective superspace}

Having derived the correct component action in the rigid limit, we must now include all of the effects of supergravity. The component action from curved projective superspace is

$$
S=-\frac{1}{2 \pi} \oint_{\mathcal{C}} v_{i}^{+} \mathrm{d} v^{i+} \int \mathrm{d}^{4} x e \mathcal{L}^{--}+\frac{1}{2 \pi} \oint_{\mathcal{C}} v_{i}^{-} \mathrm{d} v^{i-} \int \mathrm{d}^{4} x e \mathcal{L}^{++},
$$

where

$$
\begin{aligned}
\mathcal{L}^{--}= & \frac{1}{16}\left(\nabla^{-}\right)^{2}\left(\bar{\nabla}^{-}\right)^{2} \mathcal{F}^{++}-\frac{i}{8}\left(\bar{\psi}_{m}^{-} \bar{\sigma}^{m}\right)^{\alpha} \nabla_{\alpha}^{-}\left(\bar{\nabla}^{-}\right)^{2} \mathcal{F}^{++}-\frac{i}{8}\left(\psi_{m}^{-} \sigma^{m}\right)_{\dot{\alpha}} \bar{\nabla}^{\dot{\alpha}-}\left(\nabla^{-}\right)^{2} \mathcal{F}^{++} \\
& +\frac{1}{4}\left(\left(\psi_{n}^{-} \sigma^{n m}\right)^{\alpha} \bar{\psi}_{m}^{\dot{\alpha}-}+\psi_{n}{ }^{\alpha-}\left(\bar{\sigma}^{n m} \bar{\psi}_{m}^{-}\right)^{\dot{\alpha}}-i \mathcal{V}_{m}^{--}\left(\sigma^{m}\right)_{\alpha \dot{\alpha}}\right)\left[\nabla_{\alpha}^{-}, \bar{\nabla}_{\dot{\alpha}}^{-}\right] \mathcal{F}^{++} \\
& +\frac{1}{4}\left(\psi_{m}^{-} \sigma^{m n} \psi_{n}^{-}\right)\left(\nabla^{-}\right)^{2} \mathcal{F}^{++}+\frac{1}{4}\left(\bar{\psi}_{m}^{-} \bar{\sigma}^{m n} \bar{\psi}_{n}^{-}\right)\left(\bar{\nabla}^{-}\right)^{2} \mathcal{F}^{++} \\
& -\left(\frac{1}{2} \epsilon^{m n p q}\left(\psi_{m}^{-} \sigma_{n} \bar{\psi}_{p}^{-}\right) \psi_{q}^{\alpha-}-2\left(\psi_{m}^{-} \sigma^{m n}\right)^{\alpha} \mathcal{V}_{n}^{--}\right) \nabla_{\alpha}^{-} \mathcal{F}^{++} \\
& +\left(\frac{1}{2} \epsilon^{m n p q}\left(\bar{\psi}_{m}^{-} \bar{\sigma}_{n} \psi_{p}^{-}\right) \bar{\psi}_{q \dot{\alpha}}^{-}-2\left(\bar{\psi}_{m}^{-} \bar{\sigma}^{m n}\right)_{\dot{\alpha}} \mathcal{V}_{n}^{--}\right) \bar{\nabla}^{\dot{\alpha}-} \mathcal{F}^{++} \\
& +3 \epsilon^{m n p q}\left(\psi_{m}^{-} \sigma_{n} \bar{\psi}_{p}^{-}\right) \mathcal{V}_{q}^{--} \mathcal{F}^{++}, \\
\mathcal{L}^{++}= & -\left[3 D+4 f_{a}{ }^{a}-4\left(\bar{\psi}_{m}^{-} \bar{\sigma}^{m n} \hat{\bar{\phi}}_{n}^{+}\right)+4\left(\psi_{m}^{-} \sigma^{m n} \hat{\phi}_{n}^{+}\right)-3 \epsilon^{m n p q}\left(\psi_{m}^{-} \sigma_{n} \bar{\psi}_{p}^{-}\right) \mathcal{V}_{q}^{++}\right] \mathcal{F}^{++} \\
& +\left[\frac{3}{2} \chi^{\alpha+}-i\left(\bar{\phi}_{m}^{+} \bar{\sigma}^{m}\right)^{\alpha}+2\left(\psi_{m}^{-} \sigma^{m n}\right)^{\alpha} \mathcal{V}_{n}^{++}\right] \nabla_{\alpha}^{-} \mathcal{F}^{++} \\
& -\left[\frac{3}{2} \chi_{\dot{\alpha}}^{+}-i\left(\phi_{m}^{+} \sigma^{m}\right)_{\dot{\alpha}}+2\left(\bar{\psi}_{m}^{-} \bar{\sigma}^{m n}\right)_{\dot{\alpha}} \mathcal{V}_{n}^{++}\right] \bar{\nabla}^{\dot{\alpha}-} \mathcal{F}^{++} \\
& -\frac{i}{4} \mathcal{V}_{m}^{++}\left(\bar{\sigma}^{m}\right)^{\dot{\alpha} \alpha}\left[\nabla_{\alpha}^{-}, \bar{\nabla}_{\dot{\alpha}}^{-}\right] \mathcal{F}^{++} .
\end{aligned}
$$

As discussed in [18], it is always possible to complexify the auxiliary $\mathrm{SU}(2)$ manifold to $\mathrm{SL}(2, \mathbb{C})$ and then choose a contour where $u_{i}$ is constant. The resulting formulation of projective superspace is exactly that given in [19-22] and is clearly advantageous for evaluating component actions: the integral involving $\mathcal{L}^{++}$automatically vanishes.

\footnotetext{
${ }^{19} \mathrm{~A}$ proof using flat projective superspace was given in [62] for the case of a single frozen vector multiplet (see also [34, 47, 48, 63]).
} 
However, we will take an even larger shortcut which avoids the need to make any such choice. Because we are dealing with a hyperkähler cone, we can write, using (3.2),

$$
\mathcal{F}^{++}=\frac{i}{2} \Gamma_{I}^{+} \Upsilon^{I+}-\frac{i}{2} \breve{\Gamma}_{\bar{I}}^{+} \breve{\Upsilon}^{\bar{I}+}
$$

If we were to take all of the component fields to be on-shell, then $\Gamma_{I}^{+}$would be an arctic multiplet to all orders in its $\theta$ expansion. But then the superspace Lagrangian would be built out of the sum of an arctic and an antarctic superfield and such Lagrangians vanish even in curved space. The proof of this statement is quite simple (see [21] for an equivalent argument in $\mathrm{SU}(2)$ superspace) and we will review it in appendix B.

Of course, what we seek to do is to put only the auxiliary fields on-shell, so that the above argument holds only up through $\theta^{2}$. This means that we can use (6.4) to evaluate all terms except the leading ones in $\mathcal{L}^{--}$. If we write the action $S$ as

$$
\begin{aligned}
S & =-\frac{1}{2 \pi} \oint_{\mathcal{C}} v_{i}^{+} \mathrm{d} v^{i+} \int \mathrm{d}^{4} x e\left(T_{0}+T_{1}\right)+S_{\text {rest }}, \\
T_{0} & =\frac{1}{16}\left(\nabla^{-}\right)^{2}\left(\bar{\nabla}^{-}\right)^{2} \mathcal{F}^{++}, \quad T_{1}=-\frac{i}{8}\left(\bar{\psi}_{m}^{-} \bar{\sigma}^{m}\right)^{\alpha} \nabla_{\alpha}^{-}\left(\bar{\nabla}^{-}\right)^{2} \mathcal{F}^{++}+\text {h.c. },
\end{aligned}
$$

we will find that all contributions to the component action arise solely from $T_{0}$ and $T_{1}$. There will be additional remainder terms within $T_{0}$ and $T_{1}$ that involve only the combination $\Gamma_{I}^{+} \Upsilon^{I+}$ and its conjugate; when combined with $S_{\text {rest }}$, which also depends solely on this combination, all these terms will turn out to vanish.

One final point: to avoid confusing the CKV $\chi^{\mu}$ of the target space with the spinor field $\chi_{\alpha i}$ of the conformal supergravity multiplet, we will from now on always arrange to lower the target space index of the CKV so that we deal instead with $K_{\mu}=\left(K_{\mathrm{a}}, K_{\overline{\mathrm{a}}}\right) \equiv \chi_{\mu}$ or we will rewrite it as $A_{i}{ }^{\mathrm{a}}=\chi^{\mu} f_{\mu i}{ }^{\mathrm{a}}$.

\subsection{Remaining evaluation of $T_{0}$}

The first term $T_{0}$ is the most complicated as it must generate all the interactions present even in the rigid supersymmetric theory. For this reason, we have already performed the majority of its evaluation in the previous section. We again can decompose $T_{0}$ as in (5.2), replacing gauged covariant derivatives with supergravity covariant derivatives. Taking all the terms that contributed in the rigid limit, we found

$$
\begin{aligned}
T_{0} \sim & \frac{i}{v^{\underline{1}+} v^{2}+}\left[\frac{1}{2} \nabla^{\dot{\alpha} \alpha} \phi^{\mathrm{a}} \nabla_{\alpha \dot{\alpha}} \phi^{\overline{\mathrm{b}}} g_{\mathrm{a} \overline{\mathrm{b}}}-\frac{i}{4} g_{\mathrm{b} \overline{\mathrm{b}}}\left(\zeta^{\alpha \mathrm{b}} \overleftrightarrow{\nabla}_{\alpha \dot{\alpha}} \bar{\zeta}^{\dot{\alpha} \overline{\mathrm{b}}}\right)+\frac{1}{16} \zeta^{\mathrm{a}} \zeta^{\mathrm{b}} \bar{\zeta}^{\overline{\mathrm{a}}} \bar{\zeta}^{\overline{\mathrm{b}}} R_{\mathrm{a} \overline{\mathrm{a}} \overline{\mathrm{b}}}\right. \\
& +Y_{i j}^{r} D_{r}^{i j}+\left(\lambda_{i}^{\alpha r} \Omega_{r \alpha}^{i}+\text { h.c. }\right)-\frac{1}{2} W^{r} \bar{W}^{s} J_{r}^{\mu} J_{s}^{\nu} g_{\mu \nu} \\
& \left.-\frac{1}{4} W^{r} \zeta^{\mathrm{a}} \zeta^{\mathrm{b}} \nabla_{\mathrm{a}}\left(\omega_{\mathrm{bc}} J_{r}^{\mathrm{c}}\right)-\frac{1}{4} \bar{W}^{s} \bar{\zeta}^{\overline{\mathrm{a}}} \bar{\zeta}^{\overline{\mathrm{b}}} \nabla_{\mathrm{a}}\left(\omega_{\overline{\mathrm{b}} \overline{\mathrm{c}}} J_{r}^{\overline{\mathrm{c}}}\right)\right] \\
& +\nabla^{\dot{\alpha} \alpha}\left[\frac{1}{2} z_{1}^{--} \Gamma_{I}^{+} \nabla_{\alpha}^{-} \bar{\nabla}_{\dot{\alpha}}^{-} \Upsilon^{I+}+\frac{i}{2}\left(z_{1}^{--}\right)^{2} \Gamma_{I}^{+} \nabla^{\dot{\alpha} \alpha} \Upsilon^{I+}\right. \\
& \left.+\frac{1}{4} z_{1}^{--} \nabla_{\alpha}^{-} \Gamma_{I}^{+} \bar{\nabla}_{\dot{\alpha}}^{-} \Upsilon^{I+}-\frac{1}{4} z_{1}^{--} \bar{\nabla}_{\dot{\alpha}}^{-} \Gamma_{I}^{+} \nabla_{\alpha}^{-} \Upsilon^{I+}-\text { a.t. }\right]
\end{aligned}
$$


up to terms involving covariant supergravity fields. The reader may check that all of the steps followed in the rigid case to derive this expression follow equally well here. Now we must include the covariant supergravity fields. The steps required to derive these terms are quite similar to those used for the covariant fields of the vector multiplet, so we will omit an explicit discussion. Finally, it will be convenient to rewrite

$$
\frac{1}{2} \nabla^{\alpha \dot{\alpha}} \phi^{\mathrm{a}} \nabla_{\alpha \dot{\alpha}} \phi^{\overline{\mathrm{b}}} g_{\mathrm{a} \overline{\mathrm{b}}}=\frac{1}{4} \nabla^{\dot{\alpha} \alpha} \nabla_{\alpha \dot{\alpha}} K+\frac{1}{2} K_{\mu} \widehat{\nabla}_{a} \nabla^{a} \phi^{\mu}
$$

where $\widehat{\nabla}_{a}$ carries the target space connection. (This formula is valid when $K$ describes a hyperkähler cone.) After this rewriting, we find

$$
\begin{aligned}
T_{0}= & \frac{i}{v^{\underline{1}+} v^{2+}}\left[\frac{1}{2} K_{\mu} \widehat{\square} \phi^{\mu}-\frac{i}{4} g_{\mathrm{b} \overline{\mathrm{b}}}\left(\zeta^{\alpha \mathrm{b}} \overleftrightarrow{\widehat{\nabla}}_{\alpha \dot{\alpha}} \bar{\zeta}^{\dot{\alpha} \overline{\mathrm{b}}}\right)+\frac{1}{16} \zeta^{\mathrm{a}} \zeta^{\mathrm{b}} \bar{\zeta}^{\overline{\mathrm{a}}} \bar{\zeta}^{\overline{\mathrm{b}}} R_{\mathrm{a} \overline{\mathrm{a}} \mathrm{b}}\right. \\
& +Y_{i j}^{r} D_{r}^{i j}+\left(\lambda_{i}^{\alpha r} \Omega_{r \alpha}^{i}+\text { h.c. }\right)-\frac{1}{2} W^{r} \bar{W}^{s} J_{r}^{\mu} J_{s}^{\nu} g_{\mu \nu} \\
& -\frac{1}{4} W^{r} \zeta^{\mathrm{a}} \zeta^{\mathrm{b}} \nabla_{\mathrm{a}}\left(\omega_{\mathrm{bc}} J_{r}^{\mathrm{c}}\right)-\frac{1}{4} \bar{W}^{r} \bar{\zeta}^{\overline{\mathrm{a}}} \bar{\zeta}^{\overline{\mathrm{b}}} \nabla_{\mathrm{a}}\left(\omega_{\overline{\mathrm{b}} \overline{\mathrm{c}}} J_{r}^{\overline{\mathrm{c}}}\right) . \\
& \left.-\frac{3}{2} D K-\frac{1}{4}\left(W^{\alpha \beta} \zeta_{\beta}^{\mathrm{b}} \zeta_{\alpha}^{\mathrm{a}} \omega_{\mathrm{ba}}+\text { h.c. }\right)-\frac{3}{4}\left(\bar{\chi}_{\dot{\alpha}}^{j} \bar{\nabla}_{j}^{\dot{\alpha}} K+\text { h.c. }\right)\right] \\
& +\frac{3}{2}\left[i z_{1}^{--} \bar{\chi}_{\dot{\alpha}}^{-} \bar{\nabla}^{\dot{\alpha}-}\left(\Gamma_{I}^{+} \Upsilon^{I+}\right)-\frac{i}{2}\left(z_{1}^{--}\right)^{2} \bar{\chi}_{\dot{\alpha}}^{+} \bar{\nabla}^{\dot{\alpha}-}\left(\Gamma_{I}^{+} \Upsilon^{I+}\right)-(\operatorname{antarctic} \text { term })+\text { h.c. }\right] \\
& +\nabla^{\dot{\alpha} \alpha} B_{\alpha \dot{\alpha}}^{--}+D^{--} B .
\end{aligned}
$$

In the final line, we have collected a total covariant derivative, with

$$
\begin{aligned}
B_{\alpha \dot{\alpha}}^{--}= & z_{1}^{--}\left(\frac{1}{2} \Gamma_{I}^{+} \nabla_{\alpha}^{-} \bar{\nabla}_{\dot{\alpha}}^{-} \Upsilon^{I+}+\frac{1}{4} \nabla_{\alpha} \Gamma_{I}^{+} \bar{\nabla}_{\dot{\alpha}} \Upsilon^{I+}-\frac{1}{4} \bar{\nabla}_{\dot{\alpha}} \Gamma_{I}^{+} \nabla_{\alpha} \Upsilon^{I+}\right) \\
& +\frac{i}{2}\left(z_{1}^{--}\right)^{2} \Gamma_{I}^{+} \nabla^{\dot{\alpha} \alpha} \Upsilon^{I+} \text { - a.t. }+\frac{1}{4} \frac{i}{v^{\underline{1}+} v^{\underline{2}}+} \nabla_{\alpha \dot{\alpha}} K
\end{aligned}
$$

and a total contour derivative with

$$
\begin{aligned}
B= & -\frac{3 i}{2} z_{1}^{--} D \Upsilon^{I+} \Gamma_{I}^{+}+\frac{3 i}{2} z_{2}^{--} D \breve{\Upsilon}^{\bar{I}+} \breve{\Gamma}_{\bar{I}}^{+} \\
& -\frac{3}{4}\left(i z_{1}^{--} \bar{\chi}_{\dot{\alpha}}^{+} \bar{\nabla}^{\dot{\alpha}-}\left(\Gamma_{I}^{+} \Upsilon^{I+}\right)-i z_{2}^{--} \bar{\chi}_{\dot{\alpha}}^{+} \bar{\nabla}^{\dot{\alpha}-}\left(\breve{\Gamma}_{\bar{I}}^{+} \breve{\Upsilon}^{\bar{I}+}\right)+\text { h.c. }\right)
\end{aligned}
$$

Because $B$ is not holomorphic, it cannot be dropped.

Before moving on to evaluate the first set $T_{1}$ of gravitino terms, we must address what to do with $\nabla^{\dot{\alpha} \alpha} B_{\alpha \dot{\alpha}}^{--}$. Because the vector derivative contains a number of connections including supersymmetry, $S$-supersymmetry, and $\mathrm{SU}(2)$ which are quite non-trivial - it does not vanish identically and must be separately analyzed. 


\subsection{Simplification of the total covariant derivative}

Let us denote the total covariant derivative by $T_{0 . \mathrm{TD}}=\nabla^{\dot{\alpha} \alpha} B_{\alpha \dot{\alpha}}^{--}$. This can be rewritten as

$$
\begin{aligned}
T_{0 . \mathrm{TD}}= & -\frac{1}{2} \psi^{\dot{\alpha} \alpha \beta}{ }_{j} \nabla_{\beta}^{j} B_{\alpha \dot{\alpha}}^{--}-\frac{1}{2} \psi^{\dot{\alpha} \alpha}{ }_{\dot{\beta}}{ }^{j} \bar{\nabla}_{j}^{\dot{\beta}} B_{\alpha \dot{\alpha}}^{--} \\
& -\mathcal{V}^{\dot{\alpha} \alpha--} D^{++} B_{\alpha \dot{\alpha}}^{--}+D^{--}\left(\mathcal{V}^{\dot{\alpha} \alpha++} B_{\alpha \dot{\alpha}}^{--}\right) \\
& -\frac{1}{2} \phi^{\dot{\alpha} \alpha \beta j} S_{\beta j} B_{\alpha \dot{\alpha}}^{--}-\frac{1}{2} \phi^{\dot{\alpha} \alpha}{ }_{\dot{\beta} j} \bar{S}^{\dot{\beta} j} B_{\alpha \dot{\alpha}}^{--}-f^{\dot{\alpha} \alpha b} K_{b} B_{\alpha \dot{\alpha}}^{--} \\
& +2 T_{m n}{ }^{c} e_{c}{ }^{n} B^{a--} e_{a}{ }^{m}-2 e^{-1} \partial_{m}\left(e B^{a--} e_{a}{ }^{m}\right) .
\end{aligned}
$$

Note that the last line contains an actual total derivative (which can be discarded) and a torsion term that arises from the gravitino dependence of the spin connection.

Before addressing this, let us recall the guiding principle discussed at the beginning of this section. The superfield $\Gamma_{I}^{+}$is arctic to order $\theta^{2}$, so any expressions involving at most two spinor derivatives can be treated more simply than those involving three or more. Therefore, we will separate the first line of $T_{0 . T D}$, which involves gravitinos and up to three spinor derivatives, and denote it by $T_{0 . \mathrm{TD} \mid \mathrm{Q}}$. We will analyze it shortly, but first let us discuss what happens to the remaining terms. Denote these by $T_{0 . \mathrm{TD} \mid \mathrm{rest}}$. Assuming that $\Gamma_{I}^{+}$is an arctic superfield to this order, $B_{\alpha \dot{\alpha}}^{--}$can be written as

$$
\begin{aligned}
B_{\alpha \dot{\alpha}}^{--}= & \frac{1}{4} z_{1}^{--} \nabla_{\alpha}^{-} \bar{\nabla}_{\dot{\alpha}}^{-}\left(\Gamma_{I}^{+} \Upsilon^{I+}\right)+\frac{i}{4}\left(z_{1}^{--}\right)^{2} \nabla_{\alpha \dot{\alpha}}\left(\Gamma_{I}^{+} \Upsilon^{I+}\right)-(\operatorname{antarctic} \text { term }) \\
& +D^{--}\left(\frac{1}{8} \frac{1}{v^{\underline{1}+} v^{\underline{2}}+} \nabla_{\alpha}^{+} \bar{\nabla}_{\dot{\alpha}}^{+} K\right)
\end{aligned}
$$

We emphasize that this equation holds only if we do not apply any further spinor derivatives. Now when we insert this expression into $T_{0 . T D \mid r e s t}$, the last term of $B_{\alpha \dot{\alpha}}^{--}$will vanish as it leads to a total contour derivative of a holomorphic quantity. This means that the contribution of $T_{0 . T D \mid r e s t}$ to the contour integral can be taken as

$$
\begin{aligned}
T_{0 . \mathrm{TD} \mid \mathrm{rest}}= & -\mathcal{V}^{\dot{\alpha} \alpha--} D^{++} \hat{B}_{\alpha \dot{\alpha}}^{--}+D^{--}\left(\mathcal{V}^{\dot{\alpha} \alpha++} \hat{B}_{\alpha \dot{\alpha}}^{--}\right)-f^{\dot{\alpha} \alpha b} K_{b} \hat{B}_{\alpha \dot{\alpha}}^{--} \\
& -\frac{1}{2} \phi^{\dot{\alpha} \alpha \beta j} S_{\beta j} \hat{B}_{\alpha \dot{\alpha}}^{--}-\frac{1}{2} \phi^{\dot{\alpha} \alpha}{ }_{\dot{\beta} j} \bar{S}^{\dot{\beta} j} \hat{B}_{\alpha \dot{\alpha}}^{--}+2 e_{a}{ }^{m} T_{m n}{ }^{c} e_{c}{ }^{n} \hat{B}^{a--}, \\
\hat{B}_{\alpha \dot{\alpha}}^{--}= & \frac{1}{4} z_{1}^{--} \nabla_{\alpha}^{-} \bar{\nabla}_{\dot{\alpha}}^{-}\left(\Gamma_{I}^{+} \Upsilon^{I+}\right)+\frac{i}{4}\left(z_{1}^{--}\right)^{2} \nabla_{\alpha \dot{\alpha}}\left(\Gamma_{I}^{+} \Upsilon^{I+}\right)-(\text { antarctic term }) .
\end{aligned}
$$

The important feature of $T_{0 . \mathrm{TD} \mid \text { rest }}$ is that it depends only on $\Gamma_{I}^{+} \Upsilon^{I+}$ and its antarctic conjugate. For now we set these aside and focus on the terms first order in the gravitino.

\subsection{Gravitino terms}

Now we must address terms involving a single explicit gravitino field. There are two: $T_{0 . \mathrm{TD} \mid \mathrm{Q}}$ arises from the total covariant derivative $\nabla^{\dot{\alpha} \alpha} B_{\alpha \dot{\alpha}}^{--}$and $T_{1}$ arises from the original action. First we will need several formulae for the spinor derivatives of $B_{\alpha \dot{\alpha}}^{--}$. There are a number of ways to potentially organize the resulting expression. We wish to construct as much as possible expressions involving the arctic combination $\Gamma_{I}^{+} \Upsilon^{I+}$, the hyperkähler 
potential $K$ and its derivatives, and various pullbacks of the hyperkähler two-form $\Omega^{++}$. To accomplish this, it is convenient to rewrite $B_{\alpha \dot{\alpha}}^{--}$into the equivalent form

$$
\begin{aligned}
B_{\alpha \dot{\alpha}}^{--}= & \frac{1}{4} z_{1}^{--} \nabla_{\alpha}^{-} \bar{\nabla}_{\dot{\alpha}}^{-}\left(\Gamma_{I}^{+} \Upsilon^{I+}\right)+\frac{i}{4}\left(z_{1}^{--}\right)^{2} \nabla_{\alpha \dot{\alpha}}\left(\Gamma_{I}^{+} \Upsilon^{I+}\right) \\
& +\frac{1}{4} z_{1}^{--}\left(\Gamma_{I}^{+} \nabla_{\alpha}^{-} \bar{\nabla}_{\dot{\alpha}}^{-} \Upsilon^{I+}-\Upsilon^{I+} \nabla_{\alpha}^{-} \bar{\nabla}_{\dot{\alpha}}^{-} \Gamma_{I}^{+}\right)+\frac{i}{4}\left(z_{1}^{--}\right)^{2}\left(\Gamma_{I}^{+} \nabla_{\alpha \dot{\alpha}} \Upsilon^{I+}-\Upsilon^{I+} \nabla_{\alpha \dot{\alpha}} \Gamma_{I}^{+}\right) \\
& -(\text {antarctic term })+\frac{1}{4} \frac{i}{v^{\underline{1}} v^{\underline{2}}+} \nabla_{\alpha \dot{\alpha}} K
\end{aligned}
$$

The first line involves only the arctic combination $\Gamma_{I}^{+} \Upsilon^{I+}$ while the second line is manifestly antisymmetric in $\Gamma_{I}^{+}$and $\Upsilon^{I+}$ and leads to pullbacks of $\Omega^{++}$. Using relations such as

$$
\begin{aligned}
\Gamma_{I}^{+} \nabla_{\beta}^{-} \Upsilon^{I+} & =-\frac{1}{2} \nabla_{\beta}^{+} K+\frac{1}{2} \nabla_{\beta}^{-}\left(\Gamma_{I}^{+} \Upsilon^{I+}\right), \\
\nabla_{\beta}^{-}\left(\Gamma_{I}^{+} \nabla_{\alpha \dot{\alpha}} \Upsilon^{I+}\right)-\nabla_{\alpha \dot{\alpha}}\left(\Gamma_{I}^{+} \nabla_{\beta}^{-} \Upsilon^{I+}\right) & =-\Omega_{\beta}^{+} \alpha \dot{\alpha}+\Gamma_{I}^{+}\left[\nabla_{\beta}^{-}, \nabla_{\alpha \dot{\alpha}}\right] \Upsilon^{I+}
\end{aligned}
$$

one can show that

$$
\begin{aligned}
\nabla_{\beta}^{+} B_{\alpha \dot{\alpha}}^{--}= & \frac{i}{2} z_{1}^{--} \nabla_{\beta \dot{\alpha}} \nabla_{\alpha}^{-}\left(\Gamma_{I}^{+} \Upsilon^{I+}\right)-2 z_{1}^{--} \epsilon_{\beta \alpha} \bar{\lambda}_{\dot{\alpha}}^{-r} D_{r}^{++}+\left(z_{1}^{--}\right)^{2} \epsilon_{\beta \alpha} \bar{\lambda}_{\dot{\alpha}}^{+r} D_{r}^{++}-\text {a.t. } \\
& +\frac{1}{v^{\underline{1}+} v^{2}+}\left(\frac{i}{4} \nabla_{\alpha \dot{\alpha}} \nabla_{\beta}^{+} K-\frac{i}{2} \nabla_{\beta \dot{\alpha}} \nabla_{\alpha}^{+} K-\frac{i}{2} \Omega_{\alpha \beta \dot{\alpha}}^{+}\right. \\
& \left.-\frac{3}{4} \epsilon_{\beta \alpha} \bar{\chi}_{\dot{\alpha}}^{+} K+\frac{1}{4} \epsilon_{\beta \alpha} \bar{W}_{\dot{\alpha} \dot{\beta}} \bar{\nabla}^{\dot{\beta}+} K+\frac{1}{2} \epsilon_{\beta \alpha} \bar{W}^{s} \Omega_{s \dot{\alpha}}^{+}\right)
\end{aligned}
$$

and

$$
\begin{aligned}
\nabla_{\beta}^{-} B_{\alpha \dot{\alpha}}^{--}= & \frac{1}{4} z_{1}^{--} \epsilon_{\beta \alpha}\left(\nabla^{-}\right)^{2}\left(\Gamma_{I}^{+} \bar{\nabla}_{\dot{\alpha}}^{-} \Upsilon^{I+}\right)+\frac{i}{4}\left(z_{1}^{--}\right)^{2} \nabla_{\alpha \dot{\alpha}} \nabla_{\beta}^{-}\left(\Gamma_{I}^{+} \Upsilon^{I+}\right)-\frac{i}{4}\left(z_{1}^{--}\right)^{2} \nabla_{\alpha \dot{\alpha}} \nabla_{\beta}^{+} K \\
& -\frac{i}{2}\left(z_{1}^{--}\right)^{2} \Omega_{\beta \alpha \dot{\alpha}}^{+}+\frac{3}{4}\left(z_{1}^{--}\right)^{2} \epsilon_{\beta \alpha} \bar{\chi}_{\dot{\alpha}}^{+} D^{--}\left(\Gamma_{I}^{+} \Upsilon^{I+}\right)+\frac{3}{4}\left(z_{1}^{--}\right)^{2} \epsilon_{\beta \alpha} \bar{\chi}_{\dot{\alpha}}^{+} K \\
& -\frac{3}{2}\left(z_{1}^{--}\right)^{2} \epsilon_{\beta \alpha} \bar{\chi}_{\dot{\alpha}}^{-} \Gamma_{I}^{+} \Upsilon^{I+}+\frac{1}{4}\left(z_{1}^{--}\right)^{2} \epsilon_{\beta \alpha} \bar{W}_{\dot{\alpha} \dot{\beta}} \bar{\nabla}^{\dot{\beta}-}\left(\Gamma_{I}^{+} \Upsilon^{I+}\right) \\
& -\frac{1}{4}\left(z_{1}^{--}\right)^{2} \epsilon_{\beta \alpha} \bar{W}_{\dot{\alpha} \dot{\beta}} \bar{\nabla}^{\dot{\beta}+} K+\left(z_{1}^{--}\right)^{2} \epsilon_{\beta \alpha} \bar{\lambda}_{\dot{\alpha}}^{-r} D_{r}^{++}-(\operatorname{antarctic} \text { term }) \\
& +\frac{1}{v^{\underline{1}} v^{\underline{2}+}}\left(\frac{i}{4} \nabla_{\alpha \dot{\alpha}} \nabla_{\beta}^{-} K+\frac{i}{2} \Omega_{\alpha \beta \dot{\alpha}}^{-}-\frac{3}{4} \epsilon_{\beta \alpha} \bar{\chi}_{\dot{\alpha}}^{-} K\right. \\
& \left.+\frac{1}{4} \epsilon_{\beta \alpha} \bar{W}_{\dot{\alpha} \dot{\beta}} \bar{\nabla}^{\dot{\beta}-} K+\frac{1}{2} \epsilon_{\beta \alpha} \bar{W}^{s} \Omega_{s \dot{\alpha}}^{-}\right) .
\end{aligned}
$$

It helps to rewrite $T_{1}+T_{0 . \mathrm{TD} \mid \mathrm{Q}}$ as

$$
\begin{aligned}
T_{1}+T_{0 . \mathrm{TD} \mid \mathrm{Q}}= & \frac{1}{8} \psi_{\alpha}^{\alpha \dot{\alpha}-}\left(\nabla^{-}\right)^{2}\left(\Gamma_{I}^{+} \bar{\nabla}_{\dot{\alpha}}^{-} \Upsilon^{I+}\right)-\frac{1}{4} \psi_{\alpha}^{\dot{\alpha} \alpha+} \nabla^{\beta-} B_{\beta \dot{\alpha}}^{--}+\frac{1}{4} \psi_{\alpha}^{\dot{\alpha} \alpha-} \nabla^{\beta+} B_{\beta \dot{\alpha}}^{--} \\
& -\frac{1}{2} \psi^{\dot{\alpha} \alpha \beta-} \nabla_{(\beta}^{+} B_{\alpha) \dot{\alpha}}^{--}+\frac{1}{2} \psi^{\dot{\alpha} \alpha \beta+} \nabla_{(\beta}^{-} B_{\alpha) \dot{\alpha}}^{--}+\text {h.c. }
\end{aligned}
$$


The first and second terms involve a common expression $\left(\nabla^{-}\right)^{2}\left(\Gamma_{I}^{+} \bar{\nabla}_{\dot{\alpha}}^{-} \Upsilon^{I+}\right)$ given by

$$
\begin{aligned}
\frac{1}{8}\left(\nabla^{-}\right)^{2}\left(\Gamma_{I}^{+} \bar{\nabla}^{\dot{\alpha}-} \Upsilon^{I+}\right)= & \Gamma_{I}^{+} \Xi^{I \dot{\alpha}-}-\frac{1}{2} M_{I}^{-} \Psi^{I \dot{\alpha}}-\frac{i}{4} \Psi_{\alpha}^{I} A^{I \alpha \dot{\alpha}-} \\
& +z_{1}^{--}\left(\frac{i}{4} \nabla^{\dot{\alpha} \alpha} \nabla_{\alpha}^{-}\left(\Gamma_{I}^{+} \Upsilon^{I+}\right)-\frac{1}{4} \bar{W}_{\dot{\beta}}^{\dot{\alpha}} \bar{\nabla}^{\dot{\beta}-}\left(\Gamma_{I}^{+} \Upsilon^{I+}\right)+\frac{3}{2} \bar{\chi}^{\dot{\alpha}-} \Gamma_{I}^{+} \Upsilon^{I+}\right. \\
& -\frac{3}{4} \bar{\chi}^{\dot{\alpha}+} D^{--}\left(\Gamma_{I}^{+} \Upsilon^{I+}\right)-\frac{i}{4} \nabla^{\dot{\alpha} \alpha} \nabla_{\alpha}^{+} K-\frac{i}{2} \Omega_{\alpha}^{+\dot{\alpha} \alpha} \\
& \left.+\frac{1}{4} \bar{W}_{\dot{\beta}}^{\dot{\alpha}} \bar{\nabla}^{\dot{\beta}+} K-\frac{3}{4} \bar{\chi}^{\dot{\alpha}+} K-2 \bar{\lambda}^{\dot{\alpha} r-} D_{r}^{++}\right) \\
& +\left(z_{1}^{--}\right)^{2}\left(\bar{\lambda}^{\dot{\alpha} r+} D_{r}^{++}\right) .
\end{aligned}
$$

The coefficient of this term in (6.18) involves $\psi_{\alpha}^{\alpha \dot{\alpha}-}-z_{1}^{--} \psi_{\alpha}^{\dot{\alpha} \alpha+}=\psi^{\alpha \dot{\alpha} \frac{1}{\alpha}} / v^{\underline{1}+}$ and so the first line of (6.19) contributes a purely arctic expression to (6.18) that can be discarded under the contour integral. This had to be the case as otherwise we would need to impose the explicit form for $\Xi^{I \dot{\alpha}-}$, which involves the field equation for the physical fermion. Now we can analyze the remaining terms in (6.18). Discarding a total contour derivative,

$$
\begin{aligned}
T_{1}+T_{0 . \mathrm{TD} \mid \mathrm{Q}} \sim & -\frac{1}{4} z_{1}^{--} \psi_{\alpha}^{\alpha \dot{\alpha}-} \bar{W}_{\dot{\alpha} \dot{\beta}} \bar{\nabla}^{\dot{\beta}-}\left(\Gamma_{I}^{+} \Upsilon^{I+}\right)+\frac{1}{8}\left(z_{1}^{--}\right)^{2} \psi_{\alpha}^{\alpha \dot{\alpha}+} \bar{W}_{\dot{\alpha} \dot{\beta}} \bar{\nabla}^{\dot{\beta}-}\left(\Gamma_{I}^{+} \Upsilon^{I+}\right) \\
& +\frac{3}{2} z_{1}^{--} \psi_{\alpha}^{\alpha \dot{\alpha}-} \bar{\chi}^{\dot{\alpha}-} \Gamma_{I}^{+} \Upsilon^{I+}-\frac{3}{4} z_{1}^{--} \psi_{\alpha}^{\alpha \dot{\alpha}-} \bar{\chi}^{\dot{\alpha}+} D^{--}\left(\Gamma_{I}^{+} \Upsilon^{I+}\right) \\
& -\frac{3}{4}\left(z_{1}^{--}\right)^{2} \psi_{\alpha}^{\alpha \dot{\alpha}+} \bar{\chi}^{\dot{\alpha}-} \Gamma_{I}^{+} \Upsilon^{I+}+\frac{3}{8}\left(z_{1}^{--}\right)^{2} \psi_{\alpha}^{\alpha \dot{\alpha}+} \bar{\chi}^{\dot{\alpha}+} D^{--}\left(\Gamma_{I}^{+} \Upsilon^{I+}\right) \\
& -\frac{3 i}{8} z_{1}^{--} \psi_{\alpha}^{\alpha \dot{\alpha}-} \nabla_{\beta \dot{\alpha}} \nabla^{\beta-}\left(\Gamma_{I}^{+} \Upsilon^{I+}\right)+\frac{3 i}{16}\left(z_{1}^{--}\right)^{2} \psi_{\alpha}^{\alpha \dot{\alpha}+} \nabla_{\beta \dot{\alpha}} \nabla^{\beta-}\left(\Gamma_{I}^{+} \Upsilon^{I+}\right) \\
& +\frac{i}{4}\left(\frac{1}{2}\left(z_{1}^{--}\right)^{2} \psi^{\dot{\alpha}(\alpha \beta)+}-z_{1}^{--} \psi^{\dot{\alpha}(\alpha \beta)-}\right) \nabla_{\alpha \dot{\alpha}} \nabla_{\beta}^{-}\left(\Gamma_{I}^{+} \Upsilon^{I+}\right)-\text { a.t. } \\
& +\frac{1}{4} \frac{i}{v v^{+} v_{\underline{2}}^{+}} \psi_{\alpha \dot{\alpha}}^{\alpha \dot{\alpha}}\left(\nabla_{\beta \dot{\alpha}} \nabla^{\beta j} K+\Omega_{\beta \dot{\alpha}}^{\beta j}-i \bar{W}_{\dot{\alpha} \dot{\beta}} \bar{\nabla}^{\dot{\beta} j} K+i \bar{\chi}_{\dot{\alpha}}^{j} K\right. \\
& \left.-i \bar{W}^{s} \Omega_{s \dot{\alpha}}^{j}+4 i \bar{\lambda}_{\dot{\alpha} k}^{r} D_{r}^{j k}\right)
\end{aligned}
$$

Combining all of the above results, we find

$$
\begin{aligned}
T_{0}+T_{1}= & \frac{i}{v^{1}+v^{2}+}\left[\frac{1}{2} K_{\mu} \widehat{\square} \phi^{\mu}-\frac{i}{4} g_{\mathrm{b} \overline{\mathrm{b}}}\left(\zeta^{\alpha \mathrm{b}} \overleftrightarrow{\nabla}_{\alpha \dot{\alpha}} \bar{\zeta}^{\dot{\alpha} \overline{\mathrm{b}}}\right)+\frac{1}{16} \zeta^{\mathrm{a}} \zeta^{\mathrm{b}} \bar{\zeta}^{\mathrm{a}} \bar{\zeta}^{\overline{\mathrm{b}}} R_{\mathrm{a} \overline{\mathrm{a}} \overline{\mathrm{b}}}\right. \\
& +Y_{i j}^{r} D_{r}^{i j}+\left(\lambda_{i}^{\alpha r} \Omega_{r \alpha}^{i}+\text { h.c. }\right)-\frac{1}{2} W^{r} \bar{W}^{s} J_{r}^{\mu} J_{s}^{\nu} g_{\mu \nu} \\
& -\frac{1}{4} W^{r} \zeta^{\mathrm{a}} \zeta^{\mathrm{b}} \nabla_{\mathrm{a}}\left(\omega_{\mathrm{bc}} J_{r}^{\mathrm{c}}\right)-\frac{1}{4} \bar{W}^{r} \bar{\zeta}^{\overline{\mathrm{a}}} \bar{\zeta}^{\overline{\mathrm{b}}} \nabla_{\mathrm{a}}\left(\omega_{\overline{\mathrm{b}} \overline{\mathrm{c}}} J_{r}^{\overline{\mathrm{c}}}\right) \\
& -\frac{3}{2} D K-\frac{1}{4}\left(W^{\alpha \beta} \zeta_{\beta}^{\mathrm{b}} \zeta_{\alpha}^{\mathrm{a}} \omega_{\mathrm{ba}}+\text { h.c. }\right)-\frac{3}{4}\left(\bar{\chi}_{\dot{\alpha}}^{j} \bar{\nabla}_{j}^{\dot{\alpha}} K+\text { h.c. }\right) \\
& +\frac{1}{4} \psi^{\alpha \dot{\alpha}}{ }_{\alpha j} K_{\mu} \widehat{\nabla}_{\beta \dot{\alpha}} \zeta^{\beta j \mu}+\frac{3 i}{4} \psi^{\alpha \dot{\alpha}}{ }_{\alpha j} \bar{\chi}_{\dot{\alpha}}^{j} K \\
& \left.+\frac{i}{4} \psi_{\alpha}^{\dot{\alpha} \alpha j} \bar{W}_{\dot{\alpha} \dot{\beta}} \bar{\nabla}_{j}^{\dot{\beta}} K-\frac{i}{4} \psi^{\dot{\alpha} \alpha}{ }_{\alpha j} \bar{W}^{s} \Omega_{s \dot{\alpha}}^{j}+i \psi^{\alpha \dot{\alpha}}{ }_{\alpha j} \bar{\lambda}_{\dot{\alpha} k}^{r} D_{r}^{j k}\right]+\mathscr{R}^{--} .
\end{aligned}
$$


The terms within square braces are independent of $v^{i+}$, so their contour integral can be done immediately. This actually gives the final Lagrangian $\mathcal{L}$. The component action is

$$
S=\int \mathrm{d}^{4} x e \mathcal{L}-\frac{1}{2 \pi} \oint_{\mathcal{C}} v_{i}^{+} \mathrm{d} v^{i+} \int \mathrm{d}^{4} x e \mathscr{R}^{--}+S_{\text {rest }} .
$$

In addition to the many terms $S_{\text {rest }}$ that we separated out at the start of the calculation, there are still a large number of terms left within the remainder term $\mathscr{R}^{--}$,

$$
\begin{aligned}
\mathscr{R}^{--}= & \frac{3 i}{2} z_{1}^{--} \bar{\chi}_{\dot{\alpha}}^{-} \bar{\nabla}^{\dot{\alpha}-}\left(\Gamma_{I}^{+} \Upsilon^{I+}\right)-\frac{3 i}{4}\left(z_{1}^{--}\right)^{2} \bar{\chi}_{\dot{\alpha}}^{+} \bar{\nabla}^{\dot{\alpha}-}\left(\Gamma_{I}^{+} \Upsilon^{I+}\right) \\
& +i z_{1}^{--} \psi_{a}^{\alpha-}\left(\sigma^{a b}\right)_{\alpha}^{\beta} \nabla_{b} \nabla_{\beta}^{-}\left(\Gamma_{I}^{+} \Upsilon^{I+}\right)-\frac{i}{2}\left(z_{1}^{--}\right)^{2} \psi_{a}{ }^{\alpha+}\left(\sigma^{a b}\right)_{\alpha}{ }^{\beta} \nabla_{b} \nabla_{\beta}^{-}\left(\Gamma_{I}^{+} \Upsilon^{I+}\right) \\
& +\frac{3}{2} z_{1}^{--} \psi_{\alpha}^{\alpha \dot{\alpha}-} \bar{\chi}_{\dot{\alpha}}^{-}\left(\Gamma_{I}^{+} \Upsilon^{I+}\right)-\frac{3}{4} z_{1}^{--} \psi^{\alpha \dot{\alpha}}{ }_{\alpha} \bar{\chi}_{\dot{\alpha}}^{+} D^{--}\left(\Gamma_{I}^{+} \Upsilon^{I+}\right) \\
& -\frac{3}{4}\left(z_{1}^{--}\right)^{2} \psi^{\alpha \dot{\alpha}+} \bar{\chi}_{\dot{\alpha}}^{-}\left(\Gamma_{I}^{+} \Upsilon^{I+}\right)+\frac{3}{8}\left(z_{1}^{--}\right)^{2} \psi_{\alpha}^{\alpha \dot{\alpha}} \bar{\chi}_{\dot{\alpha}}^{+} D^{--}\left(\Gamma_{I}^{+} \Upsilon^{I+}\right) \\
& -\frac{1}{4} z_{1}^{--} \psi_{\alpha}^{\alpha \dot{\alpha}-} \bar{W}_{\dot{\alpha} \dot{\beta}} \bar{\nabla}^{\dot{\beta}-}\left(\Gamma_{I}^{+} \Upsilon^{I+}\right)+\frac{1}{8}\left(z_{1}^{--}\right)^{2} \psi_{\alpha}^{\alpha \dot{\alpha}+} \bar{W}_{\dot{\alpha} \dot{\beta}} \bar{\nabla}^{\dot{\beta}-}\left(\Gamma_{I}^{+} \Upsilon^{I+}\right) \\
& -(\text { antarctic term })+\text { h.c. }+D^{--} B+T_{0 . T D \mid \text { rest }},
\end{aligned}
$$

where $B$ and $T_{0 . T D \mid r e s t}$ are given by (6.10) and (6.13). All of these are written in terms of the arctic combination $\Gamma_{I}^{+} \Upsilon^{I+}$ and its antarctic conjugate, using the relation (6.4). In fact, it turns out that all of these terms actually vanish up to a total derivative - that is,

$$
0=-\frac{1}{2 \pi} \oint_{\mathcal{C}} v_{i}^{+} \mathrm{d} v^{i+} \int \mathrm{d}^{4} x e \mathscr{R}^{--}+S_{\text {rest }} .
$$

The proof is somewhat indirect, so we postpone it until appendix B.

\subsection{Final result}

Our final component Lagrangian is

$$
\begin{aligned}
\mathcal{L}= & \frac{1}{2} K_{\mu} \widehat{\square} \phi^{\mu}-\frac{i}{4} g_{\mathrm{b} \overline{\mathrm{b}}}\left(\zeta^{\alpha \mathrm{b}} \overleftrightarrow{\widehat{\nabla}}_{\alpha \dot{\alpha}} \bar{\zeta}^{\dot{\alpha} \overline{\mathrm{b}}}\right)+\frac{1}{16} \zeta^{\mathrm{a}} \zeta^{\mathrm{b}} \bar{\zeta}^{\overline{\mathrm{a}}} \bar{\zeta}^{\overline{\mathrm{b}}} R_{\mathrm{a} \overline{\mathrm{a}} \mathrm{b}} \\
& +Y_{i j}^{r} D_{r}^{i j}+\left(\lambda_{i}^{\alpha r} \Omega_{r \alpha}^{i}+\bar{\lambda}_{\dot{\alpha}}^{i r} \Omega_{r i}^{\dot{\alpha}}\right)-\frac{1}{2} W^{r} \bar{W}^{s} J_{r}^{\mu} J_{s}^{\nu} g_{\mu \nu} \\
& -\frac{1}{4} W^{r} \zeta^{\mathrm{a}} \zeta^{\mathrm{b}} \nabla_{\mathrm{a}}\left(\omega_{\mathrm{bc}} J_{r}^{\mathrm{c}}\right)-\frac{1}{4} \bar{W}^{r} \bar{\zeta}^{\overline{\mathrm{a}}} \bar{\zeta}^{\overline{\mathrm{b}}} \nabla_{\overline{\mathrm{a}}}\left(\omega_{\overline{\mathrm{b}} \overline{\mathrm{c}}} J_{r}^{\overline{\mathrm{c}}}\right) \\
& -\frac{3}{2} D K-\frac{1}{4}\left(W^{\alpha \beta} \zeta_{\beta}^{\mathrm{b}} \zeta_{\alpha}^{\mathrm{a}} \omega_{\mathrm{ba}}+\bar{W}^{\dot{\alpha} \dot{\beta}} \bar{\zeta}_{\dot{\beta}}^{\overline{\mathrm{b}}} \bar{\zeta}_{\dot{\alpha}}^{\bar{a}} \omega_{\overline{\mathrm{b}} \overline{\mathrm{a}}}\right)-\frac{3}{4} K_{\mu}\left(\chi_{j}^{\alpha} \zeta_{\alpha}^{j \mu}+\bar{\chi}_{\dot{\alpha}}^{j} \bar{\zeta}_{j}^{\dot{\alpha} \mu}\right) \\
& +\psi^{\alpha \dot{\alpha}}{ }_{\alpha j}\left(\frac{1}{4} K_{\mu} \widehat{\nabla}_{\beta \dot{\alpha}} \zeta^{\beta j \mu}-\frac{i}{4} K_{\mu} \bar{W}_{\dot{\alpha} \dot{\beta}} \bar{\zeta}^{\dot{\beta} j \mu}+\frac{3 i}{4} \bar{\chi}_{\dot{\alpha}}^{j} K-\frac{i}{4} \bar{W}^{r} \Omega_{r \dot{\alpha}}^{j}+i \bar{\lambda}_{\dot{\alpha} k}^{r} D_{r}^{j k}\right) \\
& +\psi_{\alpha \dot{\alpha}} \dot{\alpha} j\left(\frac{1}{4} K_{\mu} \widehat{\nabla}^{\dot{\beta} \alpha} \bar{\zeta}_{\dot{\beta} j}^{\mu}+\frac{i}{4} K_{\mu} W^{\alpha \beta} \zeta_{\beta j}{ }^{\mu}+\frac{3 i}{4} \chi_{j}^{\alpha} K+\frac{i}{4} W^{r} \Omega_{r \alpha j}-i \lambda^{r \alpha k} D_{r j k}\right) .
\end{aligned}
$$

It is convenient to relabel the tensor auxiliary field as $T_{a b}^{-}=4\left(\sigma_{a b}\right)_{\alpha}{ }^{\beta} W_{\beta}{ }^{\alpha}$, in accordance with tensor calculus conventions, where $T_{a b}^{-} \equiv T_{a b}{ }^{i j} \varepsilon_{i j}$. (Note that this changes the definition of self-duality used in [42] so as to agree with component conventions.) Also, a number 
of terms can be rewritten to involve the fields $A_{i}{ }^{\mathrm{a}}$, with the result

$$
\begin{aligned}
\mathcal{L}= & \frac{1}{2} K_{\mu} \widehat{\square} \phi^{\mu}-\frac{3}{2} D K-\frac{1}{2} W^{r} \bar{W}^{s} J_{r}^{\mu} J_{s}^{\nu} g_{\mu \nu}+Y_{i j}^{r} D_{r}^{i j}+\frac{1}{16} \zeta^{\mathrm{a}} \zeta^{\mathrm{b}} \bar{\zeta}^{\overline{\mathrm{a}}} \bar{\zeta}^{\overline{\mathrm{b}}} R_{\mathrm{a} \overline{\mathrm{a}} \overline{\mathrm{b}}} \\
& -\frac{1}{4}\left(\bar{\zeta}_{\dot{\alpha}}^{\overline{\mathrm{a}}}-i\left(\psi_{m j} \sigma^{m}\right)_{\dot{\alpha}} A^{j \overline{\mathrm{a}}}\right) \times \\
& \left(i g_{\overline{\mathrm{a}} \mathrm{b}} \widehat{\nabla}^{\dot{\alpha} \alpha} \zeta_{\alpha}^{\mathrm{b}}+\frac{1}{8} \omega_{\overline{\mathrm{a}} \overline{\mathrm{b}}}\left(\bar{\sigma}^{b c} \bar{\zeta}^{\overline{\mathrm{b}}}\right)^{\dot{\alpha}} T_{b c}^{+}+3 g_{\overline{\mathrm{a}} \mathrm{b}} A_{k}^{\mathrm{b}} \bar{\chi}^{\dot{\alpha} k}+\bar{\zeta}^{\dot{\alpha} \overline{\mathrm{b}}} \bar{W}^{r} \nabla_{\overline{\mathrm{a}}}\left(\omega_{\overline{\mathrm{b}} \overline{\mathrm{c}}} J_{r}^{\overline{\mathrm{c}}}\right)-2 \bar{\lambda}^{\dot{\alpha} r k} J_{r \overline{\mathrm{a}} k}\right) \\
& -\frac{1}{4}\left(\zeta^{\alpha \mathrm{a}}-i\left(\bar{\psi}_{m}^{j} \bar{\sigma}^{m}\right)^{\alpha} A_{j}^{\mathrm{a}}\right) \times \\
& \left(i g_{\mathrm{a} \overline{\mathrm{b}}} \widehat{\nabla}_{\alpha \dot{\alpha}} \bar{\zeta}^{\dot{\alpha} \overline{\mathrm{b}}}+\frac{1}{8} \omega_{\mathrm{ab}}\left(\sigma^{b c} \zeta^{\mathrm{b}}\right)_{\alpha} T_{b c}^{-}+3 g_{\mathrm{ab}} A^{k \overline{\mathrm{b}}} \chi_{\alpha k}+\zeta_{\alpha}^{\mathrm{b}} W^{r} \nabla_{\mathrm{a}}\left(\omega_{\mathrm{bc}} J_{r}^{\mathrm{c}}\right)-2 \lambda_{\alpha k}^{r} J_{r \mathrm{a}}{ }^{k}\right) \\
& +\frac{1}{2} \zeta^{\alpha \mathrm{a}} \lambda_{\alpha i}^{r} J_{r \mathrm{a}}{ }^{i}+\frac{1}{2} \bar{\zeta}_{\dot{\alpha}}^{\overline{\mathrm{a}}} \bar{\lambda}^{r \dot{\alpha} i} J_{r \overline{\mathrm{a}} i} .
\end{aligned}
$$

Two useful checks can be made. First, in the rigid supersymmetric limit, this matches the component Lagrangian constructed from (5.39). Second, the Lagrangian must be $S$ supersymmetric. To check this, it helps to note that the parenthetical terms in the third and fifth lines, which multiply explicit gravitinos, must vanish under $S$-supersymmetry. It is straightforward to check that the remaining terms all cancel against each other.

This result can be compared with eq. (5.4) of [29], where superconformal tensor calculus conventions (see e.g. [67]) were used. The relation between those conventions and ours for conformal supergravity are spelled out in [42]; for example, one must swap the locations of $\mathrm{SU}(2)$ indices, taking care to observe that tensor calculus conventions employ $\varepsilon^{\underline{12}}=\varepsilon_{\underline{12}}=1$ while we use $\epsilon^{\underline{12}}=\epsilon_{\underline{21}}=1$. This amounts to the exchange of $\varepsilon^{i j} \rightarrow-\epsilon_{i j}$ and $\varepsilon_{i j} \rightarrow \epsilon^{i j}$. The target space conventions of [29] also differ in several ways from ours. For example, their $\operatorname{Sp}(n)$ indices $(\bar{\alpha}, \alpha)$ correspond to our $(\mathrm{a}, \bar{a})$, and we use a different normalization for the fermions. For the sigma model component fields, one must exchange

$$
\phi^{A} \rightarrow \phi^{\mu}, \quad A_{i}{ }^{\alpha} \rightarrow A^{i \overline{\mathrm{a}}}, \quad A^{i \bar{\alpha}} \rightarrow A_{i}{ }^{\mathrm{a}}, \quad \zeta^{\bar{\beta}} \rightarrow \frac{1}{2} \zeta_{\alpha}^{\mathrm{b}}, \quad \zeta^{\beta} \rightarrow \frac{1}{2} \bar{\zeta}^{\dot{\alpha} \overline{\mathrm{b}}} .
$$

The target space geometric quantities are related as

$$
\begin{aligned}
& \Omega_{\bar{\alpha} \bar{\beta}} \rightarrow \omega_{\mathrm{ab}}, \\
& G_{\bar{\alpha} \beta} \rightarrow g_{\mathrm{a} \overline{\mathrm{b}}}, \\
& \gamma_{i \bar{\alpha}}^{A} \rightarrow f_{\mathrm{a}}{ }^{i \mu}, \quad V_{A}^{i \bar{\alpha}} \rightarrow f_{\mu i}{ }^{\mathrm{a}}, \\
& \left(J^{i j}\right)^{A}{ }_{B} \rightarrow-\left(\mathcal{J}_{i j}\right)^{\mu}{ }_{\nu}, \\
& \left(k_{I}\right)^{\alpha}{ }_{\beta} \rightarrow \nabla_{\overline{\mathrm{b}}} J_{r}^{\overline{\mathrm{a}}}, \\
& P_{I}^{i j} \rightarrow D_{\text {rij }} .
\end{aligned}
$$

The components of the vector multiplet are also defined slightly differently:

$$
X^{I} \rightarrow-\frac{1}{2} W^{r}, \quad \Omega_{i}^{I} \rightarrow-\epsilon^{i j} \lambda_{\alpha j}^{r}, \quad \Omega^{I i} \rightarrow \epsilon_{i j} \bar{\lambda}^{r \dot{\alpha} j}, \quad Y_{i j}^{I} \rightarrow Y^{r i j} .
$$

Other quantities can be derived from these relations and the equations given in [29].

\section{Conclusion}

Our goal in this paper was quite specific: to recover the component action of a hyperkähler cone coupled to conformal supergravity given in [29] using projective superspace methods. Our approach was based on the formal solution of the equations (3.1), corresponding to 
the requirement that the dual multiplets $\Gamma_{I}^{+}$and $\breve{\Gamma}_{\bar{I}}^{+}$were respectively arctic and antarctic, as advocated in $[24,56]$. We have not attempted to actually solve these equations for any specific models - the formal solutions were sufficient to yield the component action - but it should be mentioned that many specific cases do admit solutions. A major class involve $\mathcal{O}(2 n)$ hypermultiplets and the generalized Legendre transform construction $[12,13,25,68]$ : here the number of auxiliary fields is finite from the outset. Another major class involve target spaces that are cotangent bundles of Hermitian symmetric spaces [56, 57, 69-73] (see also [54] for a pedagogical discussion).

As discussed in [29,34], the component action (6.26) does not directly yield the general action of canonically normalized supergravity coupled to matter. One must fix the Weyl gauge and the $\mathrm{SU}(2)_{R}$ gauge: this effectively eliminates four degrees of freedom from the hypermultiplet manifold, reducing the $4 n$-dimensional hyperkähler cone to a $4(n-$ 1)-dimensional quaternion-Kähler manifold. In addition, one must add another matter sector (e.g. vector multiplets parametrizing a special Kähler manifold) to yield a consistent equation of motion for the auxiliary $D$. This can all be understood at the component level, but what about in superspace?

Here again comparing with $\mathcal{N}=1$ superspace is helpful. Recall the geometry of an $\mathcal{N}=1$ superconformal sigma model is a Kähler cone. If the chiral superfields are reorganized into the set $\left\{\phi_{0}, \phi^{i}\right\}$ with $\chi^{\mathrm{a}}=\left(\phi_{0}, 0\right)$, then without loss of generality the Kähler cone potential can be written as ${ }^{20}$

$$
K=-3 \phi_{0} \bar{\phi}_{0} e^{-\mathcal{K} / 3}
$$

for a real function $\mathcal{K}\left(\phi^{i}, \bar{\phi}^{\bar{\imath}}\right)$, subject to the Kähler transformations,

$$
\phi_{0} \rightarrow \phi_{0} e^{F / 3}, \quad \mathcal{K} \rightarrow \mathcal{K}+F+\bar{F}, \quad F=F\left(\phi^{i}\right) .
$$

Imposing the dilatation $+\mathrm{U}(1)_{R}$ gauge $\phi_{0}=e^{\mathcal{K} / 6}$ leads to the standard formulation of an $\mathcal{N}=1$ supergravity-matter system (including a superpotential is straightforward) in the so-called Kähler superspace [74] with a simple Lagrangian

$$
-3 \int \mathrm{d}^{4} x \mathrm{~d}^{2} \theta \mathrm{d}^{2} \bar{\theta} E
$$

The function $\mathcal{K}$ is absorbed within the superspace structure and the original Kähler transformations become associated with an effective $\mathrm{U}(1)_{R}$ symmetry of the component fields. This Kähler superspace is extremely useful: for example, it eliminates the need for complicated field redefinitions to attain a canonically normalized action and even allows higher derivative interactions to be easily incorporated in a Kähler-covariant way.

It would be extremely interesting to construct the $\mathcal{N}=2$ analogue of the above superspace geometry: a proposal along the lines discussed above has already been made in [19]. Of course, there is already a harmonic superspace description of quaternionic sigma models coupled to supergravity [36-38], so finding a direct link between the projective and harmonic approaches, as was done for the hyperkähler case [27], would be enlightening. It

\footnotetext{
${ }^{20}$ The overall sign must be negative to yield the correct sign for the Einstein-Hilbert term.
} 
would presumably make use of the technology of [35], developed to address quaternionic sigma models in twistor language. It would also be nice to understand the quaternionic examples in harmonic superspace (see e.g. the quaternionic Taub-NUT [75, 76] and the general two-center metrics $[77,78])$ in this context.

We should note that the projective superspace description of quaternionic sigma models takes a particularly elegant form if the hyperkähler cone possesses an additional U(1) isometry that separately rotates the arctics and antarctics. Then it is always possible to perform a duality transformation, exchanging one polar multiplet for a tensor multiplet $\mathcal{G}^{++}$, giving the supergravity-matter action $[22,79]$

$$
-\frac{1}{2 \pi} \oint_{\mathcal{C}} \mathrm{d} \tau \int \mathrm{d}^{4} x \mathrm{~d}^{4} \theta^{+} \mathcal{E}^{--}\left(\mathcal{G}^{++} \log \left(\mathcal{G}^{++} / \mathrm{i} \Upsilon_{0}^{+} \breve{\Upsilon}_{0}^{+}\right)+\mathcal{G}^{++} K(\Upsilon, \breve{\Upsilon})\right)
$$

where $K(\Upsilon, \breve{\Upsilon})$ is a real function of weight-zero arctics $\Upsilon$ and antarctics $\breve{\Upsilon}$. (The arctic $\Upsilon_{0}^{+}$ drops out of the component action but is necessary to keep the argument of the logarithm dimensionless.) This is the natural $\mathcal{N}=2$ generalization of the $\mathcal{N}=1$ new minimal supergravity action coupling the tensor multiplet compensator to a Kähler potential. The component Lagrangian of (7.4) gives the general supergravity-matter system (after gaugefixing) involving a quaternion-Kähler target space arising from a hyperkähler cone with a triholomorphic $\mathrm{U}(1)$ isometry.

An interesting open question would be to address hypermultiplet couplings in the presence of various higher derivative terms. Higher derivative tensor multiplet actions were discussed at the component level in [80]; their lift to projective superspace (and generalization to include $\mathcal{O}(2 n)$ multiplets) was addressed in [81]. In principle, one should be able to apply the latter construction to include higher derivative actions for off-shell polar multiplets. However, the elimination of the auxiliaries in the presence of such terms becomes (even formally) a formidable process. Nevertheless, superspace would seem to be the natural mechanism for constructing such terms in a systematic way, and the calculation undertaken here is an important first step. It would be intriguing to explore these issues further.

\section{Acknowledgments}

I am grateful to Sergei Kuzenko, Ulf Lindström, Martin Roček, and Gabriele TartaglinoMazzucchelli for valuable comments and suggestions. This work was supported in part by the ERC Advanced Grant no. 246974, "Supersymmetry: a window to non-perturbative physics" and by the European Commission Marie Curie International Incoming Fellowship grant no. PIIF-GA-2012-627976.

\section{A Vector multiplet conventions and supersymmetry transformations}

The introduction of a gauge connection to the curved projective superspace [18] is straightforward and can be found in [20,22]. We introduce a superspace connection $\mathcal{A}_{\underline{M}}=\mathcal{A}_{\underline{M}}{ }^{r} X_{r}$, with $X_{r}$ the formal gauge generator which acts on a field $\Psi$ in a given representation as 
$X_{r} \Psi=\mathbf{T}_{r} \Psi$ for matrices $\mathbf{T}_{r}$. Note that the algebra of the operators possesses a different sign from the algebra of the matrices,

$$
\left[X_{r}, X_{s}\right]=-f_{r s}{ }^{t} X_{t} \quad \Longrightarrow \quad\left[\mathbf{T}_{r}, \mathbf{T}_{s}\right]=+f_{r s}{ }^{t} \mathbf{T}_{t}
$$

For a compact gauge group, $\mathbf{T}_{r}$ are anti-Hermitian matrices, and so are frequently defined with an additional factor of $i$ to make them Hermitian. For a non-linear sigma model with gauged isometries, the scalars $\phi^{\mu}=\left(\phi^{\mathrm{a}}, \bar{\phi}^{\overline{\mathrm{a}}}\right)$ transform as $X_{r} \phi^{\mu}=J_{r}{ }^{\mu}$ into a Killing vector $J_{r}{ }^{\mu}$. The fermions $\zeta_{\alpha}^{\mathrm{b}}$ and $\bar{\zeta}_{\dot{\alpha}}^{\bar{b}}$ transform as

$$
X_{r} \zeta_{\alpha}^{\mathrm{b}}=\zeta_{\alpha}^{\mathrm{c}} \partial_{\mathrm{c}} J_{r}^{\mathrm{b}}, \quad X_{r} \bar{\zeta}_{\dot{\alpha}}^{\overline{\mathrm{b}}}=\bar{\zeta}_{\dot{\alpha}}^{\overline{\mathrm{c}}} \partial_{\overline{\mathrm{c}}} J_{r}^{\overline{\mathrm{b}}}
$$

The gauge covariant derivative $\nabla_{\underline{A}}$ is defined implicitly by

$$
\partial_{\underline{M}}=E_{\underline{M}}^{\underline{A}} \nabla_{\underline{A}}+\frac{1}{2} \Omega_{\underline{M}}^{a b} M_{b a}+A_{\underline{M}} \mathbb{A}+B_{\underline{M}} \mathbb{D}+F_{\underline{M}}^{A} K_{A}+\mathcal{A}_{\underline{M}}^{r} X_{r} .
$$

The algebra of the SU(2) covariant derivatives $\nabla^{ \pm \pm}$and $\nabla^{0}$ with themselves and with the other operators remains unchanged. Similarly, the algebra of spinor covariant derivatives obeys the integrability conditions $\left\{\nabla_{\underline{\alpha}}^{ \pm}, \nabla_{\underline{\beta}}^{ \pm}\right\}=0$, which implies the dimension- 1 curvatures

$$
\left\{\nabla_{\alpha}^{ \pm}, \bar{\nabla}_{\dot{\beta}}^{\mp}\right\}=\mp 2 i \nabla_{\alpha \dot{\beta}}, \quad\left\{\nabla_{\alpha}^{ \pm}, \nabla_{\beta}^{\mp}\right\}= \pm 2 \epsilon_{\alpha \beta} \overline{\mathcal{W}}, \quad\left\{\bar{\nabla}^{\dot{\alpha} \pm}, \bar{\nabla}^{\dot{\beta} \mp}\right\}= \pm 2 \epsilon^{\dot{\alpha} \dot{\beta}} \mathcal{W}
$$

Now the curvature operator $\mathcal{W}$ receives a new contribution $W^{r} X_{r}$,

$$
\mathcal{W}=\frac{1}{2} W^{\alpha \beta} M_{\beta \alpha}+\frac{1}{4} \nabla^{\beta+} W_{\beta}{ }^{\alpha} S_{\alpha}^{-}-\frac{1}{4} \nabla^{\beta-} W_{\beta}{ }^{\alpha} S_{\alpha}^{+}+\frac{1}{4} \nabla^{\dot{\alpha} \beta} W_{\beta}{ }^{\alpha} K_{\alpha \dot{\alpha}}+W^{r} X_{r}
$$

and similarly for its complex conjugate. The new superfield $W^{r}$ is the covariant non-abelian vector multiplet. It is chiral, inert under the $\mathrm{SU}(2)$ covariant derivatives, and obeys the Bianchi identity $\left(\nabla^{+}\right)^{2} W^{r}=\left(\bar{\nabla}^{+}\right)^{2} \bar{W}^{r}$. The dimension-3/2 curvatures

$$
\left[\nabla_{\beta}^{ \pm}, \nabla_{\alpha \dot{\alpha}}\right]=-2 \epsilon_{\beta \alpha} \overline{\mathcal{W}}_{\dot{\alpha}}^{ \pm}, \quad\left[\bar{\nabla}_{\dot{\beta}}^{ \pm}, \nabla_{\alpha \dot{\alpha}}\right]=-2 \epsilon_{\dot{\beta} \dot{\alpha}} \mathcal{W}_{\alpha}^{ \pm}
$$

receive a new contribution from the gauge sector,

$$
\mathcal{W}_{\alpha}^{ \pm} \ni-\frac{i}{2} \nabla_{\alpha}^{ \pm} W^{r} X_{r}, \quad \overline{\mathcal{W}}_{\dot{\alpha}}^{ \pm} \ni-\frac{i}{2} \bar{\nabla}_{\dot{\alpha}}^{ \pm} \bar{W}^{r} X_{r}
$$

Finally, the dimension- 2 curvatures $\left[\nabla_{b}, \nabla_{a}\right]=-\mathcal{F}_{b a}$ include a contribution $\mathcal{F}_{b a}{ }^{r} X_{r}$, with

$$
\mathcal{F}_{b a}^{r}=-\frac{1}{4}\left(\sigma_{b a}\right)^{\alpha \beta}\left(\nabla_{(\alpha}^{+} \nabla_{\beta)}^{-} W^{r}-2 W_{\alpha \beta} \bar{W}^{r}\right)-\frac{1}{4}\left(\bar{\sigma}_{b a}\right)^{\dot{\alpha} \dot{\beta}}\left(\bar{\nabla}_{(\dot{\alpha}}^{+} \bar{\nabla}_{\dot{\beta})}^{-} \bar{W}^{r}-2 \bar{W}_{\dot{\alpha} \dot{\beta}} W^{r}\right) .
$$

We also use the symbol $W^{r}$ to denote the lowest component of the vector multiplet. The other covariant components of the vector multiplet are given by

$$
\lambda_{\alpha i}^{r}=\frac{1}{2} \epsilon_{i j} \nabla_{\alpha}^{j} W^{r}, \quad \bar{\lambda}^{r \dot{\alpha} i}=-\frac{1}{2} \epsilon^{i j} \bar{\nabla}_{j}^{\dot{\alpha}} \bar{W}^{r}, \quad Y^{r i j}=\frac{1}{4} \nabla^{i j} W^{r},
$$


while the component one-form $A_{m}{ }^{r}$ and two-form $F_{m n}{ }^{r}$ are given by the projections of the corresponding superspace quantities $\mathcal{A}_{m}{ }^{r}$ and $\mathcal{F}_{m n}{ }^{r}$. The supersymmetry and $S$ supersymmetry transformations are given by

$$
\begin{aligned}
\delta A_{m}{ }^{r} & =i\left(\xi_{i} \sigma^{m} \bar{\lambda}^{i r}\right)-\epsilon^{i j}\left(\xi_{i} \psi_{m j}\right) \bar{W}^{r}+\text { h.c. } \\
\delta W^{r} & =2 \xi_{i} \lambda^{i r} \\
\delta \lambda_{\alpha i}^{r} & =\left(\sigma^{a b} \xi_{i}\right)_{\alpha}\left(\mathcal{F}_{a b}{ }^{r}+\frac{1}{8} T_{a b}^{-} \bar{W}^{r}\right)-Y_{i}^{j} \xi_{\alpha j}+\frac{1}{2} \xi_{\alpha i} \bar{W}^{s} W^{t} f_{t s}{ }^{r}+i \nabla_{\alpha \dot{\beta}} W^{r} \bar{\xi}^{\dot{\beta} j}-2 \eta_{\alpha i} W^{r}, \\
\delta Y^{r i j} & =2 i \xi^{\alpha i} \nabla_{\alpha \dot{\alpha}} \bar{\lambda}_{j}^{\dot{\alpha} r}-\xi^{\alpha i} \lambda_{\alpha}^{j s} \bar{W}^{t} f_{t s}{ }^{r}-2 i \bar{\xi}_{\dot{\alpha}}{ }^{i} \nabla^{\dot{\alpha} \alpha} \lambda_{\alpha}^{j r}-\bar{\xi}_{\dot{\alpha}}{ }^{i} \bar{\lambda}^{\dot{\alpha} j s} W^{t} f_{t s}{ }^{r} .
\end{aligned}
$$

The transformation law of the gaugino involves the supercovariant curvature tensor $\mathcal{F}_{a b}{ }^{r}$, whose component form is given by

$$
\begin{aligned}
e_{m}{ }^{a} e_{n}{ }^{b} \mathcal{F}_{a b}{ }^{r}:= & F_{m n}{ }^{r}-\frac{i}{2}\left(\psi_{m j} \sigma_{n} \bar{\lambda}^{j r}-\psi_{n j} \sigma_{m} \bar{\lambda}^{j r}\right)-\frac{i}{2}\left(\bar{\psi}_{m}{ }^{j} \bar{\sigma}_{n} \lambda_{j}^{r}-\bar{\psi}_{n}{ }^{j} \bar{\sigma}_{m} \lambda_{j}^{r}\right) \\
& +\frac{1}{2} \epsilon^{i j}\left(\psi_{m i} \psi_{n j}\right) \bar{W}^{r}-\frac{1}{2} \epsilon_{i j}\left(\bar{\psi}_{m}{ }^{i} \bar{\psi}_{n}{ }^{j}\right) W^{r}
\end{aligned}
$$

\section{B Vanishing of a pure arctic action and the remainder terms in eq. (6.22)}

We begin this appendix by reviewing an important lemma: the projective superspace action

$$
-\frac{1}{2 \pi} \oint_{\mathcal{C}} \mathrm{d} \tau \int \mathrm{d}^{4} x \mathrm{~d}^{4} \theta^{+} \mathcal{E}^{--} \Lambda^{++}
$$

vanishes if $\Lambda^{++}$is a purely arctic (or antarctic) superfield. A nearly identical statement was established in [21] for the choice $\Lambda^{++}=\mathcal{G}^{++} \Lambda$ for $\mathcal{G}^{++}$a tensor multiplet and $\Lambda$ an arctic (or antarctic) superfield; the proof is exactly the same so let's briefly review it.

As discussed in [18], it is possible to complexify the auxiliary manifold $\mathrm{SU}(2)$ to $\mathrm{SL}(2, \mathbb{C})$, taking $v^{i+}=v^{i}$ and $v_{i}^{-}=u_{i} /(v, u)$. This modifies the component Lagrangian only by a total derivative. In particular, we may choose $u_{i}$ to be a fixed coordinate so long as $(v, u) \neq 0$ along the contour $\mathcal{C}$. This has the benefit of eliminating the second integral in (6.1), and so significantly simplifies the evaluation of component actions. This approach is exactly the formulation of projective superspace presented in [19-22].

Let us suppose now that $\Lambda^{++}$is arctic. Following [21], we may choose $u_{i}=(1,0)$, so that $v_{i}^{-}=(1,0) / v^{\underline{1}+}$ while $v^{i+}=v^{\underline{1}+}(1, \zeta)$. Examining the component Lagrangian (6.2) with $\mathcal{F}^{++}=\Lambda^{++}$, it is immediately apparent that $\mathcal{L}^{--}$is a purely arctic expression. That is, after factoring out a common factor of $1 /\left(v^{\underline{1}+}\right)^{2}$, the remaining terms are all free of singularities at $\zeta=0$. Such a Lagrangian exactly vanishes under the contour integral.

This is the most direct proof, but not the only one. One could also remain with the real SU(2) manifold and explicitly analyze the component reduction of (B.1). This would require defining the component fields of $\Lambda^{++}$as we did for $\Upsilon^{I+}$ in section 4.1 and then proceeding to massage each term of the component action meticulously until everything vanished. This approach is clearly much more laborious.

It turns out that this more difficult approach actually has an excellent use. If we recall the situation in section 6 , we argued that the projective superspace Lagrangian 
$\mathcal{F}^{++}=\frac{i}{2} \Gamma_{I}^{+} \Upsilon^{I+}+$ h.c. is the sum of an arctic and antarctic piece up to the $\theta^{2}$ level without imposing dynamical equations of motion. Then in analyzing the component action of $\mathcal{F}^{++}$, we stopped at (6.22) once everything had been reduced to the physical component fields or to expressions involving $\Gamma_{I}^{+} \Upsilon^{I+}$ and its complex conjugate. These we isolated into a remainder term $\mathscr{R}^{--}$, given in (6.23), as well as the terms $S_{\text {rest }}$ neglected in (6.5). All of these residual terms involved $\Gamma_{I}^{+} \Upsilon^{I+}$ (and its conjugate) with at most two spinor derivatives. We claimed that the sum of all these terms vanished. Now we will provide a proof. We will start by analyzing the component action of (B.1) using the real $\mathrm{SU}(2)$ manifold, proceeding systematically order by order in the number of gravitinos and other connection fields. We will stop after eliminating the leading terms: what remains will be those terms left over in (6.22) after choosing $\Lambda^{++}=\frac{i}{2} \Gamma_{I}^{+} \Upsilon^{I+}$, which is indeed arctic up to the $\theta^{2}$ level. These must vanish, of course, because the original action (B.1) vanishes.

We begin anew with the action (B.1) and evaluate the component Lagrangian (6.2) with $\mathcal{F}^{++}=\Lambda^{++}$. As before, we will organize the calculation as in (6.5), taking the leading terms $T_{0}$ and $T_{1}$ and placing the others into $S_{\text {rest }}$. The leading term $T_{0}$, involves the highest component of $\Lambda^{++}$. This can be decomposed as

$$
\begin{aligned}
\left(\nabla^{-}\right)^{4} \Lambda^{++}= & P^{--}-\frac{i}{2} z_{1}^{--} \nabla^{\dot{\alpha} \alpha} \nabla_{\alpha}^{-} \bar{\nabla}_{\dot{\alpha}}^{-} \Lambda^{++}+\frac{1}{2}\left(z_{1}^{--}\right)^{2} \nabla^{\dot{\alpha} \alpha} \nabla_{\alpha \dot{\alpha}} \Lambda^{++} \\
& +\frac{3}{2} z_{1}^{--} \chi^{\alpha+} D^{--} \nabla_{\alpha}^{-} \Lambda^{++}-\frac{3}{2} z_{1}^{--} \bar{\chi}_{\dot{\alpha}}^{+} D^{--} \bar{\nabla}_{\dot{\alpha}}^{-} \Lambda^{++} \\
& -\frac{3}{2} z_{1}^{--} \chi^{\alpha-} \nabla_{\alpha}^{-} \Lambda^{++}+\frac{3}{2} z_{1}^{--} \bar{\chi}_{\dot{\alpha}}^{-} \bar{\nabla}_{\dot{\alpha}}^{-} \Lambda^{++} \\
& -3 z_{1}^{--} D D^{--} \Lambda^{++}+3\left(z_{1}^{--}\right)^{2} D \Lambda^{++}
\end{aligned}
$$

where $P^{--}$is a purely arctic expansion. This can be discarded under the contour and so the relevant contributions to $T_{0}$ are

$$
\begin{aligned}
T_{0}= & -3 z_{1}^{--} \chi^{\alpha-} \nabla_{\alpha}^{-} \Lambda^{++}+\frac{3}{2}\left(z_{1}^{--}\right)^{2} \chi^{\alpha+} \nabla_{\alpha}^{-} \Lambda^{++}+\text {h.c. } \\
& +\nabla^{\dot{\alpha} \alpha} B_{\alpha \dot{\alpha}}^{--}+D^{--}\left(\frac{3}{2} z_{1}^{--} \chi^{\alpha+} \nabla_{\alpha}^{-} \Lambda^{++}+\text {h.c. }-3 z_{1}^{--} D \Lambda^{++}\right)
\end{aligned}
$$

with

$$
B_{\alpha \dot{\alpha}}^{--}=-\frac{i}{2} z_{1}^{--} \nabla_{\alpha}^{-} \bar{\nabla}_{\dot{\alpha}}^{-} \Lambda^{++}+\frac{1}{2}\left(z_{1}^{--}\right)^{2} \nabla_{\alpha \dot{\alpha}} \Lambda^{++} .
$$

Denoting the contribution of the $B_{\alpha \dot{\alpha}}^{--}$to $T_{0}$ as $T_{0 . T D}$, we evaluate it as in section 6.2 ,

$$
\begin{aligned}
T_{1}+T_{0 . \mathrm{TD} \mid \mathrm{Q}}= & -\frac{i}{8}\left(\psi_{\alpha}^{\dot{\alpha} \alpha-}-z_{1}^{--} \psi_{\alpha}^{\dot{\alpha} \alpha+}\right)\left(\nabla^{-}\right)^{2} \bar{\nabla}_{\dot{\alpha}}^{-} \Lambda^{++} \\
& -\frac{1}{4}\left(z_{1}^{--} \psi_{\alpha}^{\dot{\alpha} \alpha-}+\frac{1}{2}\left(z_{1}^{--}\right)^{2} \psi_{\alpha}^{\dot{\alpha} \alpha+}\right) \nabla_{\beta \dot{\alpha}} \nabla^{\beta-} \Lambda^{++} \\
& -\frac{3 i}{2}\left(z_{1}^{--}\right)^{2} \psi_{\alpha}^{\dot{\alpha} \alpha+}\left(\bar{\chi}_{\dot{\alpha}}^{-} \Lambda^{++}-\frac{1}{2} \bar{\chi}_{\dot{\alpha}}^{+} D^{--} \Lambda^{++}\right) \\
& +\frac{i}{4}\left(z_{1}^{--}\right)^{2} \psi_{\alpha}^{\dot{\alpha} \alpha+} \bar{W}_{\dot{\alpha} \dot{\beta}} \bar{\nabla}^{\dot{\beta}-} \Lambda^{++} \\
& -\frac{1}{2} z_{1}^{--} \psi^{\dot{\alpha}(\alpha \beta)-} \nabla_{\beta \dot{\alpha}} \nabla_{\alpha}^{-} \Lambda^{++}+\frac{1}{4}\left(z_{1}^{--}\right)^{2} \psi^{\dot{\alpha}(\alpha \beta)+} \nabla_{\beta \dot{\alpha}} \nabla_{\alpha}^{-} \Lambda^{++}+\text {h.c. }
\end{aligned}
$$


In the first line, we require the expression

$$
\begin{aligned}
\frac{1}{8}\left(\nabla^{-}\right)^{2} \bar{\nabla}^{\dot{\alpha}-} \Lambda^{++}= & \Xi^{\dot{\alpha}-}+z_{1}^{--}\left(\frac{i}{2} \nabla^{\dot{\alpha} \beta} \nabla_{\beta}^{-} \Lambda^{++}-\frac{1}{2} \bar{W}_{\dot{\beta}}^{\dot{\alpha}} \bar{\nabla}^{\dot{\beta}-} \Lambda^{++}\right. \\
& \left.+3 \bar{\chi}^{\dot{\alpha}-} \Lambda^{++}-\frac{3}{2} \bar{\chi}^{\dot{\alpha}+} D^{--} \Lambda^{++}\right) .
\end{aligned}
$$

The contribution of $\Xi^{\dot{\alpha}-}$ to the action is purely arctic so we can discard it, leaving

$$
\begin{aligned}
T_{1}+T_{0 . \mathrm{TD} \mid \mathrm{Q}}= & \left(z_{1}^{--} \psi_{\alpha}^{\dot{\alpha} \alpha-}-\frac{1}{2}\left(z_{1}^{--}\right)^{2} \psi_{\alpha}^{\dot{\alpha} \alpha+}\right) \times \\
& \left(\frac{i}{2} \bar{W}_{\dot{\alpha} \dot{\beta}} \bar{\nabla}^{\dot{\beta}-} \Lambda^{++}-3 i \bar{\chi}_{\dot{\alpha}}^{-} \Lambda^{++}+\frac{3 i}{2} \bar{\chi}_{\dot{\alpha}}^{+} D^{--} \Lambda^{++}\right) \\
& +\left(2 z_{1}^{--} \psi_{a}^{-} \sigma^{a b}-\left(z_{1}^{--}\right)^{2} \psi_{a}^{+} \sigma^{a b}\right)^{\alpha} \nabla_{b} \nabla_{\alpha}^{-} \Lambda^{++}+\text {h.c. }
\end{aligned}
$$

for the terms involving a single gravitino. Now we define $\mathscr{R}^{--}$as everything that has not yet canceled out from $T_{0}$ and $T_{1}$ :

$$
\begin{aligned}
\mathscr{R}^{--}= & -3 z_{1}^{--} \chi^{\alpha-} \nabla_{\alpha}^{-} \Lambda^{++}+\frac{3}{2}\left(z_{1}^{--}\right)^{2} \chi^{\alpha+} \nabla_{\alpha}^{-} \Lambda^{++} \\
& +\left(z_{1}^{--} \psi_{\alpha}^{\dot{\alpha} \alpha-}-\frac{1}{2}\left(z_{1}^{--}\right)^{2} \psi_{\alpha}^{\dot{\alpha} \alpha+}\right)\left(\frac{i}{2} \bar{W}_{\dot{\alpha} \dot{\beta}} \bar{\nabla}^{\dot{\beta}-} \Lambda^{++}-3 i \bar{\chi}_{\dot{\alpha}}^{-} \Lambda^{++}+\frac{3 i}{2} \bar{\chi}_{\dot{\alpha}}^{+} D^{--} \Lambda^{++}\right) \\
& +\left(2 z_{1}^{--}\left(\psi_{a}^{-} \sigma^{a b}\right)^{\alpha}-\left(z_{1}^{--}\right)^{2}\left(\psi_{a}^{+} \sigma^{a b}\right)^{\alpha}\right) \nabla_{b} \nabla_{\alpha}^{-} \Lambda^{++}+\text {h.c. }+T_{0 . T D \mid \text { rest }} \\
& +D^{--}\left(\frac{3}{2} z_{1}^{--} \chi^{\alpha+} \nabla_{\alpha}^{-} \Lambda^{++}-\frac{3}{2} z_{1}^{--} \bar{\chi}_{\dot{\alpha}}^{+} \bar{\nabla}^{\dot{\alpha}-} \Lambda^{++}-3 z_{1}^{--} D \Lambda^{++}\right)
\end{aligned}
$$

When combined with the terms in $S_{\text {rest }}$, this must vanish up to a total derivative.

Note that $\Lambda^{++}$now has at most two spinor derivatives acting on it, so we can choose $\Lambda^{++}=\frac{i}{2} \Gamma_{I}^{+} \Upsilon^{I+}$ to be arctic without any difficulty. This exactly matches the arctic part of (6.23). The part of the action $S_{\text {rest }}$ that did not involve $T_{0}$ and $T_{1}$ is also identical. But we know that all these contributions must vanish, and so it follows that the unevaluated terms in (6.22) involving $\Gamma_{I}^{+} \Upsilon^{I+}$ all cancel out. The antarctic ones also vanish in like fashion.

Lest the reader find this proof too indirect, it should be added that we have explicitly checked that the remaining terms do indeed vanish. To do so is fairly involved and requires the contributions from both integrals in (6.1). To demonstrate some of the manipulations that occur, we will show here how the cancellation occurs when all fermionic terms are turned off. The relevant bosonic terms remaining in $\mathcal{L}^{--}$are

$$
\begin{aligned}
\mathcal{L}^{--} \sim & -3 D^{--}\left(z_{1}^{--} D \Lambda^{++}\right)-\frac{i}{4} \mathcal{V}_{m}{ }^{--}\left(\bar{\sigma}^{m}\right)_{\alpha \dot{\alpha}}\left[\nabla^{\alpha-}, \bar{\nabla}^{\dot{\alpha}-}\right] \Lambda^{++} \\
& -\mathcal{V}^{\dot{\alpha} \alpha--} D^{++} B_{\alpha \dot{\alpha}}^{--}+D^{--}\left(\mathcal{V}^{\dot{\alpha} \alpha++} B_{\alpha \dot{\alpha}}^{--}\right)-f^{\dot{\alpha} \alpha b} K_{b} B_{\alpha \dot{\alpha}}^{--} \\
= & -D^{--}\left(\frac{i}{2} z_{1}^{--} \mathcal{V}^{\dot{\alpha} \alpha++} \nabla_{\alpha}^{-} \bar{\nabla}_{\dot{\alpha}}^{-} \Lambda^{++}-\frac{1}{2}\left(z_{1}^{--}\right)^{2} \mathcal{V}^{\dot{\alpha} \alpha++} \nabla_{\alpha \dot{\alpha}} \Lambda^{++}\right. \\
& \left.+3 z_{1}^{--} D \Lambda^{++}+4 z_{1}^{--} f_{a}^{a} \Lambda^{++}\right) .
\end{aligned}
$$


Those contributing to $\mathcal{L}^{++}$are given by

$$
\mathcal{L}^{++}=-\left(3 D+4 f_{a}^{a}\right) \Lambda^{++}-\frac{i}{2} \mathcal{V}^{\dot{\alpha} \alpha++} \nabla_{\alpha}^{-} \bar{\nabla}_{\dot{\alpha}}^{-} \Lambda^{++},
$$

which can be rewritten as

$$
\begin{aligned}
\mathcal{L}^{++}= & -D^{++}\left(\frac{i}{2} z_{1}^{--} \mathcal{V}^{\dot{\alpha} \alpha++} \nabla_{\alpha}^{-} \bar{\nabla}_{\dot{\alpha}}^{-} \Lambda^{++}-\frac{1}{2}\left(z_{1}^{--}\right)^{2} \mathcal{V}^{\dot{\alpha} \alpha++} \nabla_{\alpha \dot{\alpha}} \Lambda^{++}\right. \\
& \left.+3 z_{1}^{--} D \Lambda^{++}+4 z_{1}^{--} f_{a}^{a} \Lambda^{++}\right) .
\end{aligned}
$$

The combination of $\mathcal{L}^{--}$and $\mathcal{L}^{++}$is a total contour derivative and can be discarded. Similar cancellations occur with the fermionic terms, but these require much more work.

Open Access. This article is distributed under the terms of the Creative Commons Attribution License (CC-BY 4.0), which permits any use, distribution and reproduction in any medium, provided the original author(s) and source are credited.

\section{References}

[1] L. Álvarez-Gaumé and D.Z. Freedman, Geometrical Structure and Ultraviolet Finiteness in the Supersymmetric G-model, Commun. Math. Phys. 80 (1981) 443 [INSPIRE].

[2] L. Álvarez-Gaumé and D.Z. Freedman, Potentials for the Supersymmetric Nonlinear б-model, Commun. Math. Phys. 91 (1983) 87 [InSPIRE].

[3] J. Bagger and E. Witten, Matter Couplings in $N=2$ Supergravity, Nucl. Phys. B 222 (1983) 1 [inSPIRE].

[4] B. Zumino, Supersymmetry and Kähler Manifolds, Phys. Lett. B 87 (1979) 203 [InSPIRE].

[5] E. Witten and J. Bagger, Quantization of Newton's Constant in Certain Supergravity Theories, Phys. Lett. B 115 (1982) 202 [INSPIRE].

[6] Z. Komargodski and N. Seiberg, Comments on Supercurrent Multiplets, Supersymmetric Field Theories and Supergravity, JHEP 07 (2010) 017 [arXiv: 1002.2228] [INSPIRE].

[7] N. Seiberg, Modifying the Sum Over Topological Sectors and Constraints on Supergravity, JHEP 07 (2010) 070 [arXiv: 1005.0002] [INSPIRE].

[8] J. Distler and E. Sharpe, Quantization of Fayet-Iliopoulos Parameters in Supergravity, Phys. Rev. D 83 (2011) 085010 [arXiv:1008.0419] [INSPIRE].

[9] S. Hellerman and E. Sharpe, Sums over topological sectors and quantization of Fayet-Iliopoulos parameters, Adv. Theor. Math. Phys. 15 (2011) 1141 [arXiv:1012.5999] [INSPIRE].

[10] A.S. Galperin, E.A. Ivanov, V.I. Ogievetsky and E.S. Sokatchev, Harmonic Superspace, Cambridge University Press, Cambridge U.K. (2001).

[11] A. Galperin, E. Ivanov, S. Kalitsyn, V. Ogievetsky and E. Sokatchev, Unconstrained $N=2$ Matter, Yang-Mills and Supergravity Theories in Harmonic Superspace,

Class. Quant. Grav. 1 (1984) 469 [InSPIRE].

[12] A. Karlhede, U. Lindström and M. Roček, Selfinteracting Tensor Multiplets in $N=2$ Superspace, Phys. Lett. B 147 (1984) 297 [InSPIRE]. 
[13] U. Lindström and M. Roček, New HyperKähler Metrics and New Supermultiplets, Commun. Math. Phys. 115 (1988) 21 [InSPIRE].

[14] U. Lindström and M. Roček, $N=2$ Super Yang-Mills Theory in Projective Superspace, Commun. Math. Phys. 128 (1990) 191 [INSPIRE].

[15] A. Galperin, E. Ivanov, V. Ogievetsky and E. Sokatchev, HyperKähler Metrics and Harmonic Superspace, Commun. Math. Phys. 103 (1986) 515 [InSPIRE].

[16] A.S. Galperin, E.A. Ivanov, V.I. Ogievetsky and E. Sokatchev, Gauge Field Geometry From Complex and Harmonic Analyticities. HyperKähler Case, Annals Phys. 185 (1988) 22 [INSPIRE].

[17] A. Galperin and V. Ogievetsky, $N=2 D=4$ supersymmetric $\sigma$-models and Hamiltonian mechanics, Class. Quant. Grav. 8 (1991) 1757 [INSPIRE].

[18] D. Butter, A new approach to curved projective superspace, arXiv:1406.6235 [INSPIRE].

[19] S.M. Kuzenko, U. Lindström, M. Roček and G. Tartaglino-Mazzucchelli, $4 D N=2$ Supergravity and Projective Superspace, JHEP 09 (2008) 051 [arXiv: 0805.4683] [INSPIRE].

[20] S.M. Kuzenko, U. Lindström, M. Roček and G. Tartaglino-Mazzucchelli, On conformal supergravity and projective superspace, JHEP 08 (2009) 023 [arXiv:0905.0063] [INSPIRE].

[21] S.M. Kuzenko and G. Tartaglino-Mazzucchelli, Different representations for the action principle in $4 D N=2$ supergravity, JHEP 04 (2009) 007 [arXiv:0812.3464] [INSPIRE].

[22] S.M. Kuzenko, On $N=2$ supergravity and projective superspace: dual formulations, Nucl. Phys. B 810 (2009) 135 [arXiv:0807.3381] [INSPIRE].

[23] S.M. Kuzenko, Comments on $N=2$ supersymmetric $\sigma$-models in projective superspace, J. Phys. A 45 (2012) 095401 [arXiv:1110.4298] [INSPIRE].

[24] U. Lindström and M. Roček, Properties of hyperKähler manifolds and their twistor spaces, Commun. Math. Phys. 293 (2010) 257 [arXiv:0807.1366] [InSPIRE].

[25] N.J. Hitchin, A. Karlhede, U. Lindström and M. Roček, HyperKähler Metrics and Supersymmetry, Commun. Math. Phys. 108 (1987) 535 [INSPIRE].

[26] S. Alexandrov, B. Pioline, F. Saueressig and S. Vandoren, Linear perturbations of HyperKähler metrics, Lett. Math. Phys. 87 (2009) 225 [arXiv:0806.4620] [INSPIRE].

[27] D. Butter, Relating harmonic and projective descriptions of $N=2$ nonlinear $\sigma$-models, JHEP 11 (2012) 120 [arXiv:1206.3939] [INSPIRE].

[28] G.W. Gibbons and P. Rychenkova, Cones, triSasakian structures and superconformal invariance, Phys. Lett. B 443 (1998) 138 [hep-th/9809158] [INSPIRE].

[29] B. de Wit, B. Kleijn and S. Vandoren, Superconformal hypermultiplets, Nucl. Phys. B 568 (2000) 475 [hep-th/9909228] [INSPIRE].

[30] B. de Wit, B. Kleijn and S. Vandoren, Rigid $N=2$ superconformal hypermultiplets, Lect. Notes Phys. 524 (1999) 37 [hep-th/9808160] [INSPIRE].

[31] E. Sezgin and Y. Tanii, Superconformal $\sigma$-models in higher than two-dimensions, Nucl. Phys. B 443 (1995) 70 [hep-th/9412163] [INSPIRE].

[32] A. Swann, 'Hyper-Kähler and quaternionic Kähler geometry, Math. Ann. 289 (1991) 421.

[33] K. Galicki, Geometry of the scalar couplings in $N=2$ supergravity models, Class. Quant. Grav. 9 (1992) 27 [INSPIRE]. 
[34] B. de Wit, M. Roček and S. Vandoren, Hypermultiplets, hyperKähler cones and quaternion Kähler geometry, JHEP 02 (2001) 039 [hep-th/0101161] [INSPIRE].

[35] S. Alexandrov, B. Pioline, F. Saueressig and S. Vandoren, Linear perturbations of quaternionic metrics, Commun. Math. Phys. 296 (2010) 353 [arXiv:0810.1675] [inSPIRE].

[36] J.A. Bagger, A.S. Galperin, E.A. Ivanov and V.I. Ogievetsky, Gauging $N=2 \sigma$ Models in Harmonic Superspace, Nucl. Phys. B 303 (1988) 522 [inSPIRE].

[37] A. Galperin, E. Ivanov and O. Ogievetsky, Harmonic space and quaternionic manifolds, Annals Phys. 230 (1994) 201 [hep-th/9212155] [INSPIRE].

[38] E. Ivanov and G. Valent, Quaternionic metrics from harmonic superspace: Lagrangian approach and quotient construction, Nucl. Phys. B 576 (2000) 543 [hep-th/0001165] [INSPIRE].

[39] S.M. Kuzenko and G. Tartaglino-Mazzucchelli, Five-dimensional Superfield Supergravity, Phys. Lett. B 661 (2008) 42 [arXiv: 0710.3440] [INSPIRE].

[40] S.M. Kuzenko and G. Tartaglino-Mazzucchelli, 5D Supergravity and Projective Superspace, JHEP 02 (2008) 004 [arXiv:0712.3102] [INSPIRE].

[41] S.M. Kuzenko and G. Tartaglino-Mazzucchelli, Super-Weyl invariance in $5 D$ supergravity, JHEP 04 (2008) 032 [arXiv:0802.3953] [INSPIRE].

[42] D. Butter, $N=2$ conformal superspace in four dimensions, JHEP 10 (2011) 030 [arXiv:1103.5914] [INSPIRE].

[43] S.M. Kuzenko, On compactified harmonic/projective superspace, $5-D$ superconformal theories and all that, Nucl. Phys. B 745 (2006) 176 [hep-th/0601177] [INSPIRE].

[44] S.M. Kuzenko, On superconformal projective hypermultiplets, JHEP 12 (2007) 010 [arXiv:0710.1479] [INSPIRE].

[45] S.M. Kuzenko and G. Tartaglino-Mazzucchelli, Conformally flat supergeometry in five dimensions, JHEP 06 (2008) 097 [arXiv: 0804.1219] [INSPIRE].

[46] S.M. Kuzenko and G. Tartaglino-Mazzucchelli, Field theory in $4 D N=2$ conformally flat superspace, JHEP 10 (2008) 001 [arXiv:0807.3368] [INSPIRE].

[47] D. Butter, S.M. Kuzenko, U. Lindström and G. Tartaglino-Mazzucchelli, Extended supersymmetric $\sigma$-models in $A d S_{4}$ from projective superspace, JHEP 05 (2012) 138 [arXiv: 1203.5001] [INSPIRE].

[48] D. Butter, S.M. Kuzenko and G. Tartaglino-Mazzucchelli, Nonlinear $\sigma$-models with AdS supersymmetry in three dimensions, JHEP 02 (2013) 121 [arXiv:1210.5906] [INSPIRE].

[49] S.J. Gates Jr., A. Karlhede, U. Lindström and M. Roček, $N=1$ Superspace Components of Extended Supergravity, Class. Quant. Grav. 1 (1984) 227 [INSPIRE].

[50] S.J. Gates Jr., A. Karlhede, U. Lindström and M. Roček, $N=1$ Superspace Geometry of Extended Supergravity, Nucl. Phys. B 243 (1984) 221 [InSPIRE].

[51] J.M.F. Labastida, M. Roček, E. Sanchez-Velasco and P. Wills, $N=2$ Supergravity Action in Terms of $N=1$ Superfields, Phys. Lett. B 151 (1985) 111 [INSPIRE].

[52] J.M.F. Labastida, E. Sanchez-Velasco and P. Wills, The $N=2$ Vector Multiplet Coupled to Supergravity in $N=1$ Superspace, Nucl. Phys. B 256 (1985) 394 [INSPIRE]. 
[53] J.M.F. Labastida, E. Sanchez-Velasco and P. Wills, $N=2$ conformal supergravity in $N=1$ superspace, Nucl. Phys. B 278 (1986) 851 [INSPIRE].

[54] S.M. Kuzenko, Lectures on nonlinear $\sigma$-models in projective superspace, J. Phys. A 43 (2010) 443001 [arXiv:1004.0880] [inSPIRE].

[55] S.M. Kuzenko, $N=2$ supersymmetric $\sigma$-models and duality, JHEP 01 (2010) 115 [arXiv:0910.5771] [INSPIRE].

[56] S.J. Gates Jr. and S.M. Kuzenko, The CNM hypermultiplet nexus, Nucl. Phys. B 543 (1999) 122 [hep-th/9810137] [INSPIRE].

[57] S.J. Gates Jr. and S.M. Kuzenko, 4-D, $N=2$ supersymmetric off-shell $\sigma$-models on the cotangent bundles of Kähler manifolds, Fortsch. Phys. 48 (2000) 115 [hep-th/9903013] [INSPIRE].

[58] S.M. Kuzenko, Projective superspace as a double punctured harmonic superspace, Int. J. Mod. Phys. A 14 (1999) 1737 [hep-th/9806147] [INSPIRE].

[59] C.M. Hull, A. Karlhede, U. Lindström and M. Roček, Nonlinear $\sigma$ Models and Their Gauging in and Out of Superspace, Nucl. Phys. B 266 (1986) 1 [INSPIRE].

[60] S.M. Kuzenko, U. Lindström and R. von Unge, New supersymmetric $\sigma$-model duality, JHEP 10 (2010) 072 [arXiv: 1006.2299] [INSPIRE].

[61] J. Wess and J. Bagger, Supersymmetry and supergravity, Princeton University Press, Princeton U.S.A. (1992), pg. 259.

[62] F. Gonzalez-Rey and R. von Unge, Feynman rules in $N=2$ projective superspace. 2. Massive hypermultiplets, Nucl. Phys. B 516 (1998) 449 [hep-th/9711135] [INSPIRE].

[63] S.M. Kuzenko, On superpotentials for nonlinear $\sigma$-models with eight supercharges, Phys. Lett. B 638 (2006) 288 [hep-th/0602050] [INSPIRE].

[64] D. Butter and S.M. Kuzenko, The structure of $N=2$ supersymmetric nonlinear $\sigma$-models in $A d S_{4}, J H E P 11$ (2011) 080 [arXiv:1108.5290] [INSPIRE].

[65] G. Sierra and P.K. Townsend, The hyperkähler supersymmetric sigma model in six dimensions, Phys. Lett. B124 (1983) 497 [INSPIRE].

[66] G. Sierra and P.K. Townsend, The Gauge Invariant $N=2$ Supersymmetric $\sigma$ Model With General Scalar Potential, Nucl. Phys. B 233 (1984) 289 [inSPIRE].

[67] B. de Wit, P.G. Lauwers and A. Van Proeyen, Lagrangians of $N=2$ supergravity-matter systems, Nucl. Phys. B 255 (1985) 569 [INSPIRE].

[68] I.T. Ivanov and M. Roček, Supersymmetric $\sigma$-models, twistors and the Atiyah-Hitchin metric, Commun. Math. Phys. 182 (1996) 291 [hep-th/9512075] [INSPIRE].

[69] M. Arai and M. Nitta, Hyper-Kähler $\sigma$-models on (co)tangent bundles with $\mathrm{SO}(N)$ isometry, Nucl. Phys. B 745 (2006) 208 [hep-th/0602277] [INSPIRE].

[70] M. Arai, S.M. Kuzenko and U. Lindström, HyperKähler $\sigma$-models on cotangent bundles of Hermitian symmetric spaces using projective superspace, JHEP 02 (2007) 100 [hep-th/0612174] [INSPIRE].

[71] M. Arai, S.M. Kuzenko and U. Lindström, Polar supermultiplets, Hermitian symmetric spaces and hyperKähler metrics, JHEP 12 (2007) 008 [arXiv:0709.2633] [INSPIRE]. 
[72] S.M. Kuzenko and J. Novak, Chiral formulation for hyperKähler $\sigma$-models on cotangent bundles of symmetric spaces, JHEP 12 (2008) 072 [arXiv:0811.0218] [INSPIRE].

[73] M. Arai and F. Blaschke, Cotangent bundle over Hermitian symmetric space $E_{7} / E_{6} \times U(1)$ from projective superspace, JHEP 02 (2013) 045 [arXiv:1211.1537] [INSPIRE].

[74] P. Binetruy, G. Girardi and R. Grimm, Supergravity couplings: a geometric formulation, Phys. Rept. 343 (2001) 255 [hep-th/0005225] [INSPIRE].

[75] E. Ivanov and G. Valent, Quaternionic Taub-NUT from the harmonic space approach, Phys. Lett. B 445 (1998) 60 [hep-th/9809108] [INSPIRE].

[76] E. Ivanov and G. Valent, Harmonic space construction of the quaternionic Taub-NUT metric, Class. Quant. Grav. 16 (1999) 1039 [hep-th/9810005] [INSPIRE].

[77] P.-Y. Casteill, E. Ivanov and G. Valent, Quaternionic extension of the double Taub-NUT metric, Phys. Lett. B 508 (2001) 354 [hep-th/0104078] [INSPIRE].

[78] P.Y. Casteill, E. Ivanov and G. Valent, $\mathrm{U}(1) \times \mathrm{U}(1)$ quaternionic metrics from harmonic superspace, Nucl. Phys. B 627 (2002) 403 [hep-th/0110280] [INSPIRE].

[79] S.M. Kuzenko, U. Lindström and R. von Unge, New extended superconformal $\sigma$-models and Quaternion Kähler manifolds, JHEP 09 (2009) 119 [arXiv:0906.4393] [INSPIRE].

[80] B. de Wit and F. Saueressig, Off-shell $N=2$ tensor supermultiplets, JHEP 09 (2006) 062 [hep-th/0606148] [INSPIRE].

[81] D. Butter and S.M. Kuzenko, New higher-derivative couplings in $4 D N=2$ supergravity, JHEP 03 (2011) 047 [arXiv: 1012.5153] [INSPIRE]. 